\title{
Andean orogeny and the diversification of lowland neotropical rain forest trees: A case study in Sapotaceae
}

\author{
Julieth Serrano $^{\mathrm{a}, *}$, James E. Richardson ${ }^{\mathrm{b}, \mathrm{c}}$, Richard I. Milne ${ }^{\mathrm{d}}$, G. Ariadna Mondragon ${ }^{\mathrm{e}}$, \\ Julie A. Hawkins ${ }^{\mathrm{f}}$, Igor V. Bartish ${ }^{\mathrm{g}}$, Mailyn Gonzalez ${ }^{\mathrm{h}}$, Jérôme Chave ${ }^{\mathrm{i}}$, Santiago Madrinán ${ }^{\mathrm{e}, 1}$, \\ Dairon Cárdenas ${ }^{\mathrm{j}}$, S. Dayana Sanchez ${ }^{\mathrm{k}}$, Rocio Cortés-B ${ }^{\mathrm{k}}$, R. Toby Pennington ${ }^{\mathrm{a}, \mathrm{c}}$ \\ ${ }^{a}$ Geography Department, College of Life and Environmental Sciences, University of Exeter, Rennes Drive, Exeter EX4 4RJ, UK \\ ${ }^{\mathrm{b}}$ Facultad de Ciencias Naturales, Universidad del Rosario, Bogotá, D.C., Colombia \\ ${ }^{\mathrm{c}}$ Tropical Diversity Section, Royal Botanic Garden Edinburgh, 20a Inverleith Row, Edinburgh EH3 5LR, UK \\ ${ }^{\mathrm{d}}$ Institute of Molecular Plant Sciences, Kings Building, West Mains Road, University of Edinburgh, EH9 3JW, UK \\ e Laboratorio de Botánica y Sistemática, Universidad de los Andes, Apartado Aéreo 4976, Bogotá, D.C., Colombia \\ ${ }^{\mathrm{f}}$ School of Biological Sciences, University of Reading, Reading RG6 6BX, UK \\ ${ }^{g}$ Department of Population Ecology, Institute of Botany, Academy of Sciences, CZ-25243 Průhonice, Czech Republic \\ ${ }^{\mathrm{h}}$ Instituto Alexander von Humboldt, Avenida Paseo Bolivar (Circunvalar) 16-20, Bogotá, D.C., Colombia \\ ${ }^{\mathrm{i}}$ Laboratoire Evolution et Diversité Biologique UMR 5174, Université Paul Sabatier/CNRS, 31062 Toulouse, France \\ j Instituto Amazónico de Investigaciones Científicas Sinchi, Calle 20 \# 5-44, Bogotá, D.C., Colombia \\ ${ }^{\mathrm{k}}$ Herbario Forestal, Universidad Distrital, Carrera 5 Este \# 15-82, Bogotá D.C., Colombia
}

\section{A R T I C L E I N F O}

\section{Editor: Fabienne Marret-Davies}

\section{Keywords:}

Andes

Diversification

Neotropics

Panama land bridge

Pleistocene

Sapotaceae

Rain forest

\begin{abstract}
A B S T R A C T
Understanding how species diversify and evolve in species-rich areas like the lowland rain forest in the Neotropics is critical for conservation in times of unprecedented threats. To determine how the Andean uplift, the formation of the Panama land bridge, and Pleistocene climatic fluctuations affected dispersal and diversification in the Sapotaceae subfamily Chrysophylloideae, we collected 146 Chrysophylloideae accessions in previously under-explored areas, generating one of the most geographically complete data sets for neotropical Sapotaceae. Sapotaceae is a good model to test diversification hypotheses in lowland neotropical rain forests as it predominantly occurs $<1000 \mathrm{~m}$ altitude, and it is an abundant and species-rich group in this biome. We generated a time calibrated phylogeny of 123 Sapotaceae species based upon the nuclear ribosomal internal transcribed spacer region that suggests migration between lineages to the east and the west Andean Cordilleras occurred before and after periods of major uplift, indicating that the Andes did not represent a significant barrier to dispersal for Sapotaceae, although it may have promoted vicariance in some cases. Dispersal between South and Central America occurred mainly prior to the formation of the Panama land bridge, suggesting that this event did not affect migration patterns in Chrysophylloideae. We inferred diversification rates and detected three shifts in the phylogeny, but they are not congruent with tectonic movements during the middle Miocene and climatic changes during the Pleistocene. Finally, some species with restricted distributions appear to be phylogenetically nested within species with broader ranges, suggesting ancestor descendent relationships and insights into patterns of speciation in rain forest trees.
\end{abstract}

\section{Introduction}

The lowland rain forest biome in northern South America hosts some of the most species-rich floras in the world, and it is represented by the forests in the biogeographic units of Chocó, Amazon, Catatumbo, and
Guiana. These forests are separated from one another by three branches of the Andean mountain range (Fig. 1), and are suggested to have been formed by distinct ecological and evolutionary processes (HernándezCamacho et al., 1992; Morrone, 2001; Bernal et al., 2016; Lasso et al., 2018; Pérez-Escobar et al., 2019). These areas are considered of great

\footnotetext{
* Corresponding author at: Geography Department, College of Life and Environmental Sciences, University of Exeter, Rennes Drive, Exeter EX4 4RJ, UK.

E-mail address: juliserranoa@yahoo.com (J. Serrano).

1 Jardín Botánico de Cartagena "Guillermo Piñeres", Turbaco, Bolívar, Colombia.
} 
conservation and economic value (Bernal et al., 2016), and to strengthen local policies and prevent species extinction, it is critical to understand how their diversity is distributed and has evolved. However, due to decades of civil conflict in countries like Colombia and lack of safe access to these areas, samples from these forests have not been included in previous studies, and consequently the patterns and processes driving the distribution of this vegetation are still unclear.

Previous work suggests that dispersal and diversification patterns of plant species in the Neotropics have been affected by the uplift of the Andes e.g. Cremastoperma (Pirie et al., 2006), Dussia (Winterton et al., 2014), Orchids (Pérez-Escobar et al., 2017), palms (Bacon et al., 2018), Malvaceae (Richardson et al., 2015: Hoorn et al., 2019), Begonia
(Moonlight et al., 2015) and multiple paramo lineages (Madriñán et al., 2013). The Andean orogeny occurred in well differentiated phases (Gregory-Wodzicki, 2000; Mora et al., 2010), but it was generally initiated by major tectonic movements during the late Oligocene after the break-up of the Farallones plate, and the subsequent increment in convergence rates at the margins of the newly created Cocos and Nazca plates (Hoorn et al., 1995; Wortel, 1984). The northern part of the Andes now divides into the Eastern, Central and Western Cordilleras (Fig. 1). The Eastern Cordillera was formed as a continuous range in the middle Miocene (Winship, 1990; Hoorn et al., 1995; Albert et al., 2006), although the easternmost part (the Merida Andes) might not have reached $3000 \mathrm{~m}$ elevation until ca. 5-2 Ma, achieving their current ca.

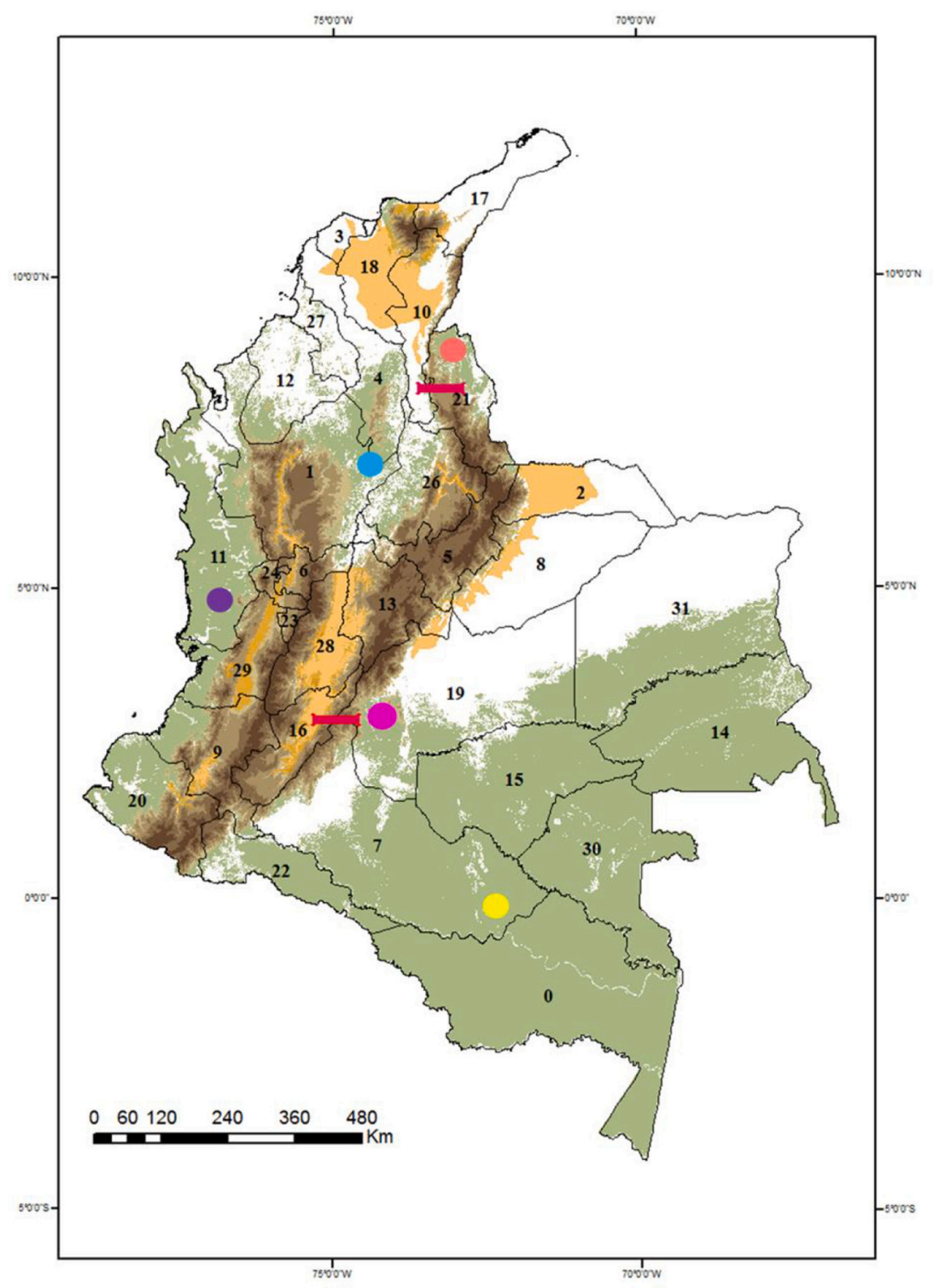

Fig. 1. Lowland rain forests of Colombia. Coloured dots indicate the fragments of lowland rain forest that can be found in Colombia. These areas are characterized by evergreen vegetation occurring under ca. $800 \mathrm{~m}$ above sea level, which is dominated by angiosperm tree species adapted to low fluctuations in temperature, high precipitation (more than $2000 \mathrm{~mm}$ per year), with high beta and alpha diversity and an abundance of lianas and epiphytes (Burnham and Johnson, 2004; IDEAM, IGAC, IAvH, et al., 2007; Reynel et al., 2013). Brown shades represent major mountain ranges, from north to south: Sierra Nevada de Santa Marta, Eastern, Western, and Central Cordilleras. Areas in yellow represent areas of dry forest. Red bars represent areas of low elevation in the Andes below $1000 \mathrm{~m}$. Black solid lines represent the administrative division of Colombia into municipalities 0: Amazonas, 1: Antioquia, 2: Arauca, 3: Atlantico, 4: Bolivar, 5: Boyaca, 6: Caldas, 7: Caquetá, 8: Casanare, 9: Cauca, 10: Cesar, 11: Chocó, 12: Cordoba, 13: Cundinamarca, 14: Guainía, 15: Guaviare, 16: Huila, 17: La Guajira, 18: Magdalena, 19: Meta, 20: Nariño, 21: Norte de Santander, 22: Putumayo, 23: Quindio, 24: Risaralda, 26: Santander, 27: Sucre, 28: Tolima, 29: Valle del Cauca, 30: Vaupes, 31: Vichada. Fragments of lowland rain forest are depicted as proposed by IDEAM et al. (2007), and dry forest areas were adapted from the WWF Ecorregions (Olson et al., 2001). (For interpretation of the references to colour in this figure legend, the reader is referred to the web version of this article.)

\section{KEY}

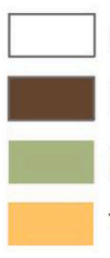

Other Biomes

Mountain forest

Lowland Rain Forest

Tropical Dry Forest
Choco

Amazon

Macarena

Catatumbo

Inter-Andean valleys

Points of low altitude 
5000 m elevation even later (Kroonenberg et al., 1990). The Central Cordillera reached its current altitude during the late Miocene at ca. 10-4 Ma (Kroonenberg et al., 1990), creating altitudinal belts that at their lower points formed the Magdalena and Cauca valleys. This orogeny was partially contemporary to the accretion of the Western Cordillera, a process that initiated during the Oligocene and early Miocene, and caused the creation of high-altitude habitats, above ca. 4000 m, during the late Miocene (Kroonenberg et al., 1990; Graham, 2011). These uplifts are presumed to have had a significant impact on biogeographic patterns, especially as a physical barrier to dispersal of lowland restricted organisms (Pirie et al., 2006; Pennington et al., 2010; Richardson et al., 2018). The Eastern Cordillera might have had the most profound effect as a barrier, because it reaches northern coastal areas, whereas the other cordilleras do not and hence could be circumvented at their northern ends (Fig. 1).

Miocene tectonic activity also generated the Panama Land Bridge (Hoorn et al., 1995; Farris et al., 2011), and together with the Andean orogeny redirected the Amazon and Orinoco rivers, which had previously emptied together into the Maracaibo basin near the northern tip of South America (Hoorn et al., 1995; Díaz de Gamero, 1996; Hoorn et al., 2010). The formation of the Panama Isthmus was followed by climatic changes during the Pleistocene with cold dry climates during glacial periods alternating with warm wet periods during interglacials.

All of these events may have altered the paleogeography or climate in northern South America, creating novel habitats, while opening or closing migration routes (Hoorn et al., 1995, 2010). Habitat fragmentation would hence have occurred at varying scales, from the large scale separation of the previously continuous lowland rain forests now separated in the Amazon, the inter-Andean valleys, and Chocó, to more local effects such as range changes during Pleistocene climatic fluctuations, and the development of drier climatic zones in inter-Andean valleys (Haffer, 1969; Prance, 1973; Richardson et al., 2001 but see Colinvaux et al., 2001; Pennington and Dick, 2004; Whinnett et al., 2005). These events may have influenced species diversification, but knowledge of how these factors affected the evolutionary history of the lowland rain forest of northern South America is far from complete.

Sapotaceae is a pantropical family of trees mainly restricted to lowland tropical rain forest, thought to have first diversified in Asia at ca. 67.1-105 Ma (Richardson et al., 2014). It comprises three subfamilies (Pennington, 1991; Faria et al., 2017), among which Chrysophylloideae is the most abundant in the Neotropics. This subfamily originated in Africa, with crown group divergence occurring around 78 $\mathrm{Ma}$, then reaching the Americas, perhaps via long distance trans-oceanic dispersal in the late Cretaceous (Bartish et al., 2011). Previous work (e.g. Armstrong et al., 2014suggested that Sapotaceae seeds may have been dispersed through trans-oceanic rafting in vegetations mats, or in the gut contents of birds. The subfamily has since become widespread and diverse in northern South America, where it is distributed almost exclusively in lowland rain forest. This restricted distribution makes it a perfect model for studying the effects of various geological and climatic processes on evolution and speciation within South American lowland rain forests.

This study aims to explore the dispersal and diversification history of Chrysophylloideae, and relate it to the geographical and climatic changes discussed above (Fig. 2). To achieve this, species of Chrysophylloideae were sampled from previously unexplored areas of lowland rain forests in northern South America. We present the first data set for neotropical Sapotaceae that includes collections from major areas of lowland rain forest on both sides of the Andes and the inter-Andean valleys. A dated phylogeny based on nuclear ribosomal internal transcribed spacer (ITS) sequences was constructed and used along with diversification rates analyses to test hypotheses of diversification, vicariance or migration caused by different geological or climatic events:

(i) if phylogenetic splits were caused by the uplift of the Eastern Cordillera of the Andes, creating habitats too high for the family and causing vicariance (Fig. 2), then splits would have increased after ca. $12 \mathrm{Ma}$ when this mountain range reached an elevation higher than about $2000 \mathrm{~m}$ (upper altitudinal limit of Sapotaceae species ranges).

(ii) if the formation of the Isthmus of Panama created a terrestrial migration route for taxa previously unable to disperse across water, then evidence for migration between South and Central
A.

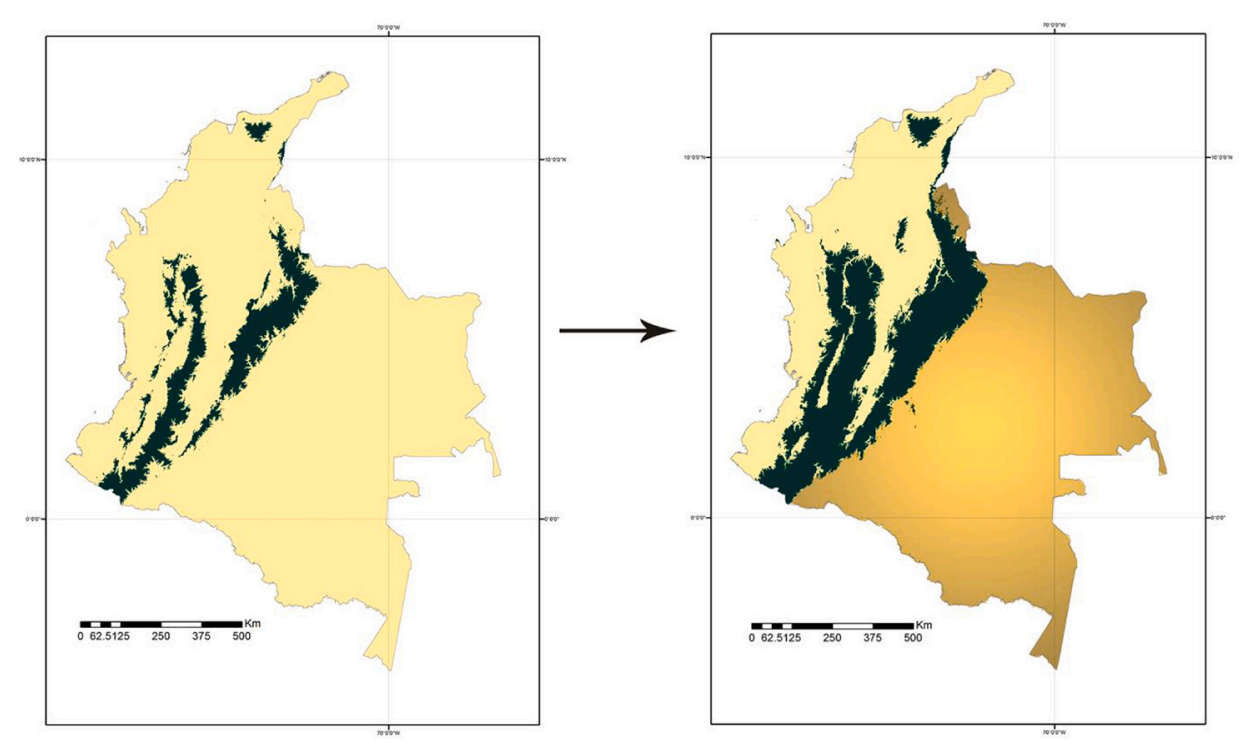

B.

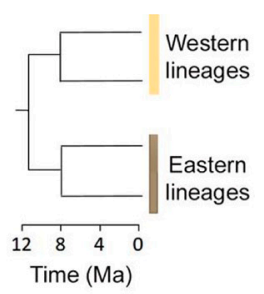

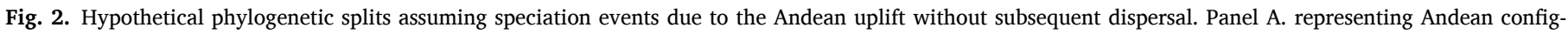

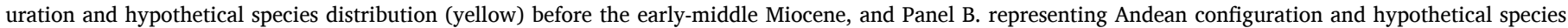

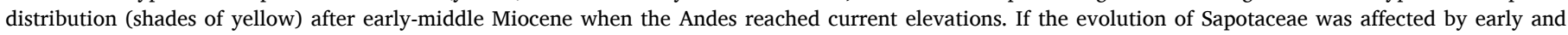

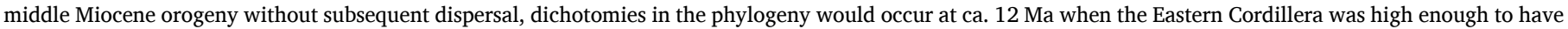
prevented migration. (For interpretation of the references to colour in this figure legend, the reader is referred to the web version of this article.) 
America would be found mainly after the uplift of the Panamanian land bridge at ca. 13-15 Ma, although the land bridge formation is thought by some to have occurred much later ( $3 \mathrm{Ma}$; Coates \& Obando, 1996).

(iii) if the uplift of the Andes affected diversification rates in Chrysophylloideae, then shifts in diversification rates would be found after the middle Miocene and during periods of major tectonic activity in northern South America.

(iv) if periodic range contractions during the Pleistocene led to diversification, then increased diversification rates would be found in this epoch.

\section{Materials and methods}

A total of 146 Chrysophylloideae accessions were collected in the previously under-explored Chocó, Magdalena Valley, Catatumbo, Amazon and Macarena lowland rain forests of Colombia, generating one of the most complete data sets in terms of geographic sampling for neotropical Sapotaceae. ITS sequence data were produced for the new collections. The remaining sequences were obtained from previous phylogenetic studies on Chrysophylloideae (Swenson et al., 2008; Gonzalez et al., 2009) and GenBank. We chose to focus our study on ITS data because it has been shown to be more variable than plastid markers (e.g. Armstrong et al., 2014), and the large amount of plastid data required to get a similar number of informative characters was beyond the scope of the present study. The full data set contains 400 accessions, with 378 individuals of neotropical Chrysophylloideae, and 22 from paleotropical Sapotaceae. Our data set represents 101 Chrysophylloideae species, $100 \%$ of the currently recognised neotropical genera, and $30 \%$ of the currently recognised species, including $71 \%$ of the species in Colombia. A list of taxa and voucher specimen information is shown in Appendix A.

\subsection{DNA isolation, amplification and sequencing}

Leaf tissue from silica-gel-dried collections was ground into a powder. Total DNA was isolated with the DNeasy ${ }^{\circledR}$ Plant Mini Kit (QIAGEN) following the manufacturer's procedures. The nuclear ITS region was amplified using primer pairs ITS5 and ITS8. PCR reactions were carried out in $20 \mu \mathrm{l}$ volume reactions by adding $2 \mu \mathrm{l}$ of dNTPs, $2 \mu \mathrm{l}$ of $10 \times \mathrm{NH} 4$ reaction buffer, $0.6 \mu \mathrm{l}$ of $\mathrm{MgCl}_{2}, 1.5 \mu \mathrm{l}$ of each primer $(10 \mu \mathrm{M}), 4 \mu \mathrm{l}$ of CES, $0.3 \mu$ l of Biotaq DNA polymerase buffer, $7.1 \mu$ d double distilled $\mathrm{H} 2 \mathrm{O}$ (ddH2O) and $1 \mu \mathrm{l}$ of DNA. The thermal cycling profile was: $3 \mathrm{~min}$ at $94{ }^{\circ} \mathrm{C}$ (denaturing) followed by 29 cycles of $1 \mathrm{~min}$ at $94{ }^{\circ} \mathrm{C}$ (denaturation), $1 \mathrm{~min}$ at $55^{\circ} \mathrm{C}$ (annealing) and $1.5 \mathrm{~min}$ at $72{ }^{\circ} \mathrm{C}$ (extension), and an additional termination step of $5 \mathrm{~min}$ at $72{ }^{\circ} \mathrm{C}$. The amplified fragments were electrophoresed on a $1 \%$ agarose gel to check for quantity. PCR products were then purified with ExoSAP-IT ${ }^{\circledR}$ (USB Corporation) which was added directly and incubated at $37^{\circ} \mathrm{C}$ for $15 \mathrm{~min}$ followed by $80{ }^{\circ} \mathrm{C}$ for $15 \mathrm{~min}$. PCR templates were used for the cycle-sequencing reactions using BigDye ${ }^{\circledR}$ Terminator v3.1 Cycle Sequencing Kit (Applied Biosystems, Inc.) and then were run on an ABI PRISM 310 sequencer (Applied Biosystems, Inc.). One of the samples in this study (Chrysophyllum colombianum) had two bands on the PCR gel. These were excised and sequenced separately (C. colombianum a and b). We detected no other examples of multiple copies of ITS in the samples used in this study.

\subsection{Sequence alignment and phylogenetic analysis}

DNA sequences were assembled and edited using Geneious 10.1.2 (Kearse et al., 2012), automatically aligned using MUSCLE and edited manually with BioEdit (Hall, 1999). Sarcosperma laurinum was used as the outgroup in all analyses as it has been shown to be the sister to all other Sapotaceae (Anderberg and Swenson, 2003). Outgroups in other families could not be used because their ITS sequences could not be aligned unambiguously with our Sapotaceae data.

Bayesian analyses were carried out with MrBayes v3.2.6 (Huelsenbeck and Ronquist, 2001; Ronquist and Huelsenbeck, 2003) through the CIPRES gateway (Miller et al., 2010). A general time reversible (GTR) model with a gamma distribution and invariant sites was identified as the best-fit substitution model in jModelTest (Darriba et al., 2012). Four independent runs of four MCMC chains were set to run for 30,000,000 cycles, sampling the Markov chain every 3000 cycles (10,000 samples) and saving branch lengths. After determining using Tracer v1.6 (Rambaut et al., 2014) that a burn-in of $25 \%$ sufficed to reach stationarity, the remaining samples were saved and used to construct a Maximum Clade Credibility tree. The average standard deviation was calculated every 30,000 generations, 1000 calculations in total. Tree probabilities were calculated and credibility intervals were set as a fraction of the highest posterior density. Clade support was represented by posterior probability (pp.) values, with weak and strong support indicated by pp. values of 50 to $95 \%$, and over $95 \%$, respectively (Swenson et al., 2008). ESS values were checked in Tracer v.1.6 and all parameters reached values $>200$.

\subsection{Divergence time estimation}

Divergence times were estimated using Bayesian dating analyses in BEAST v1.10.5 (Drummond and Rambaut, 2007). The XML (eXtensible Mark-up Language) input file was generated in BEAUti v1.10.5. The length of the chain was set to $40,000,000$ cycles, logging parameters and printing to screen every 4000 cycles. An extra run of additional $40,000,000$ cycles was set and combined with the first run to achieve ESS values higher than 200 for all parameters. A general time reversible (GTR) substitution model with a gamma distribution and invariant sites was used. Gamma categories were set to four and base frequencies were estimated. To test clock models and tree priors, Bayes Factors were calculated using path sampling and stepping-stone sampling. The clock model and tree priors that best fit our data were an uncorrelated relaxed clock with a lognormal distribution, using a speciation birth-death process. This model allows for changes in rates along the branches, and a speciation birth-death process accounts for background extinction. Priors for model parameters and statistics used a lognormal distribution for all fossil-based calibration points and a normal distribution for the secondary calibration point (Ho, 2007; Ho and Phillips, 2009).

Two fossil calibration points were used along with a secondary calibration point based on a dated phylogeny of a broader sample of asterids that utilized six well characterized fossils (Bremer et al., 2004). Psilatricolporites maculosus (Chrysophylloideae) has a more or less continuous sequence at the late Paleocene-early Eocene transition in the Maracaibo Basin in western Venezuela (Rull, 1997; Rull, 2000). The record of Psilatricolporites from Venezuela is probably the oldest reliably dated representative of Chrysophylloideae from the New World. Therefore, an offset with an age estimate of $55 \mathrm{Ma}$ was used at the crown node that included all members in the New World (node B, Fig. 3). A mean of 0.001 was set so that $95 \%$ of the intervals in the log-normal distribution would include values between the estimated age and the latest boundary of the Late Paleocene. Pollen of Psilastephanocolporites malacanthoides, reported from the upper Eocene of Nigeria (Jan du Chêne et al., 1978), was assigned to Malacantha alnifolia (synonym of the accepted name Pouteria alnifolia). The stem node of $P$. alnifolia was therefore constrained to an offset of $35 \mathrm{Ma}$ and a mean of 0.001 (node $\mathrm{C}$, Fig. 3), so that $95 \%$ of the intervals in the log-normal distribution would include values between the estimated age and the latest boundary of the Late Eocene. Finally, the crown root node of the phylogeny was dated using a secondary calibration point of $102 \mathrm{Ma}$ for the stem node of Sapotaceae, obtained from a dated phylogeny of asterids by Bremer et al. (2004) and Bremer (2009). Because we did not have any representatives of taxa that are sister to Sapotaceae in our analysis we could not recover the stem node, and therefore applied the age of the stem node of Sapotaceae to the crown root node of the phylogeny. We acknowledge that 


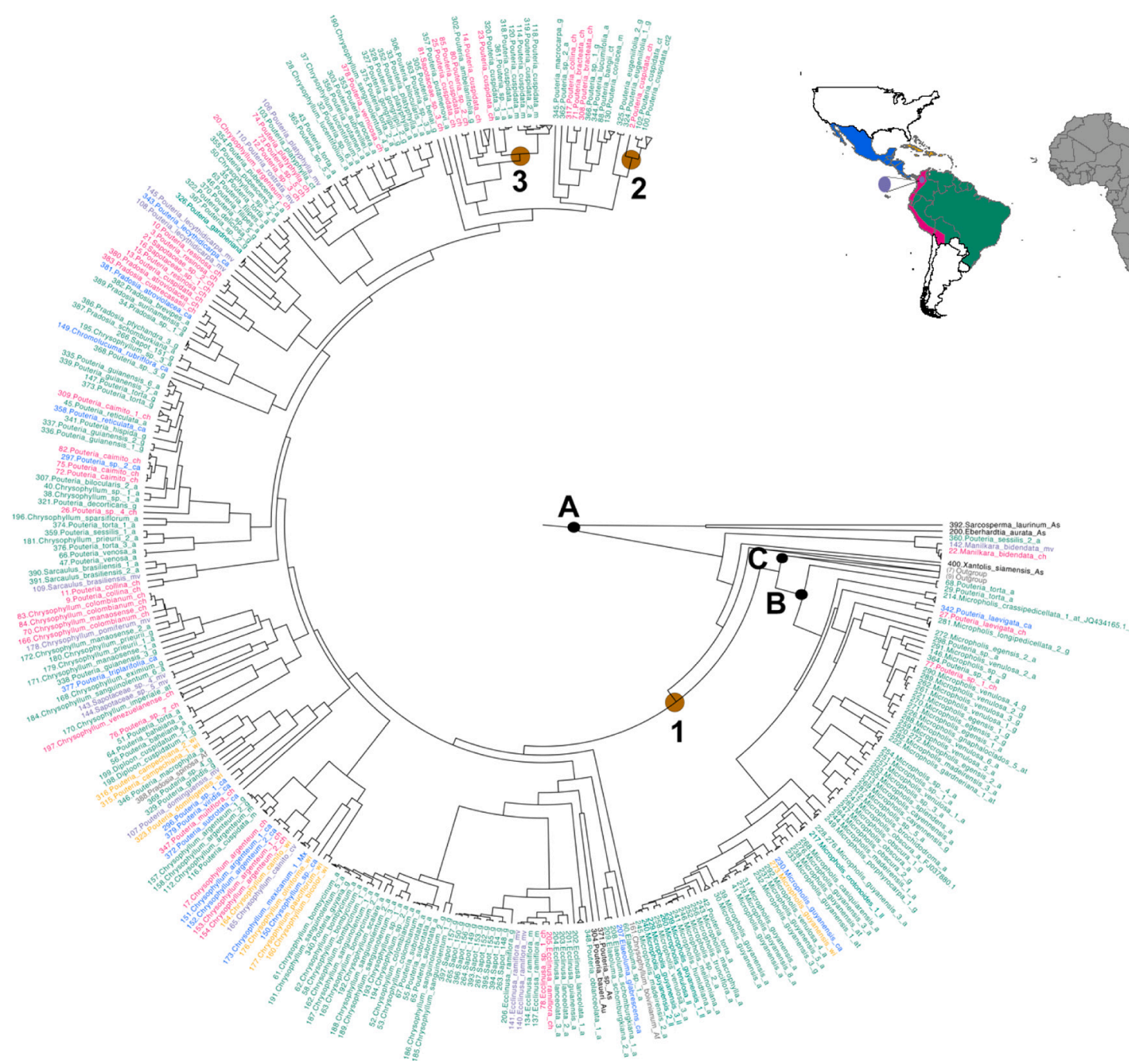

Fig. 3. Maximum clade credibility tree from the BEAST analysis indicating relationships among neotropical Sapotaceae lineages. Three calibration points are indicated: node A - age estimate of Sapotaceae following Bremer et al. (2004); node B - Neotropical pollen; node C - Malacantha alnifolia pollen. Colours in tips and the map represent in pink: areas to the west of the Eastern Cordillera, in green: areas to the east of the Eastern Cordillera, in purple: inter-Andean valleys, in blue: Central America, in yellow: West Indies, in grey: Africa, and in black: Asia. Brown circles represent shifts in diversification rates recovered by (1) BAMM 2.5, and (2 and 3) by RevBayes 10. The colour palette was chosen using the colorblindFriendly function in the R package RColorBrewer 2.0. (For interpretation of the references to colour in this figure legend, the reader is referred to the web version of this article.)

this will bias our estimates towards the older ages. The normal distribution was set with a mean of 102 and a standard deviation of three, so $95 \%$ of the intervals in a normal distribution would include the age estimated by Bremer et al. (2004) and Bremer (2009) +/ - five years (node A, Fig. 3).

\subsection{Ancestral range reconstruction}

Ancestral range reconstructions were carried out using the $\mathrm{R}$ package BioGeoBEARS v1.1.1 (Matzke Nicholas, 2018). Areas were defined based on previous evidence suggesting evolutionary distinction (Hernández-Camacho et al., 1992; Morrone, 2001; Bernal et al., 2016; Lasso et al., 2018; Pérez-Escobar et al., 2019), and on isolation due to ocean, mountain or dry climate barriers: Amazon, Atlantic Forest, Catatumbo, Guyanas, Macarena, West Indies, Central America, Chocó, Cauca Valley, Magdalena Valley, Asia, and Africa (Fig. 3). The MCC tree obtained in the BEAST analyses was used as an input tree. The maximum number of areas was set to three as this was the maximum number of areas where any given taxon was found in our phylogeny. The DEC, DIVALIKE and BAYAREALIKE models were evaluated, and the model with the lowest AIC value was selected. Models including the " $\mathrm{j}$ " parameter were not used considering the conceptual and methodological issues introduced by them (Ree and Sanmartín, 2018).

\subsection{Diversification rates}

We used BAMM 2.5.0 (Rabosky, 2014) to identify and quantify changes in evolutionary rates. BAMM uses a Markov chain Monte Carlo (MCMC) to compare evolutionary models of diversification. We ran a speciation-extinction model with a global sampling fraction of 0.3 to account for incomplete taxon sampling. Priors were set using the setBAMMpriors function in $\mathrm{R}$ ( $\mathrm{R}$ Core Team. $\mathrm{R}, 2018$ ). The analyses were run for 50,000,000 generations and 4 Markov chains. The mcmc output and the event file were written with a frequency of 5000. To analyse the output, we used the R package BAMMtools v2.1.6 (Rabosky et al., 2014). Using BAMMtools v2.1.6 we checked for convergence, computed the $95 \%$ credible set of shift configurations, and extracted the best configuration. To identify the number of rate shifts we calculated the Bayes Factor and compared among models. Changes in rates through time were plotted using the plotRateThroughTime function.

Issues related to the reliability of BAMM analyses were raised by previous studies (e.g. Rabosky, 2014; Moore et al., 2016; Meyer and 
Román-Palacios, 2018; Rabosky, 2019) but under the parameters specified for our analyses with an expected number of shifts $=1$, these issues should not hinder determination of patterns of diversification (Rabosky et al., 2017), except in cases where small rate shifts occurred. To account for that and other concerns related to BAMM, we also inferred branch specific diversification rates using RevBayes v10 (Höhna et al., 2016). RevBayes uses a MCMC under a birth-death process with diversification rates changing among branches. We used the time-calibrated phylogeny generated in BEAST as a starting tree, and the root age parameter was derived from it. The rate categories were set to six, and the effect of varying the number of rates categories from 3 to 10, was tested. To account for incomplete taxon sampling, the total number of species was set as the total number of species currently accepted for Sapotaceae. Diversification rates were calculated using the fnDiscretizeDistribution, and results were visualised using the R package RevGadgets v1.

\section{Results}

\subsection{Divergence time estimation and ancestral range reconstruction}

Analyses were performed on 400 accessions with an aligned ITS data matrix of 1151 characters. The first diverging nodes of the phylogeny are weakly supported, but posterior probability values increase for more apically placed clades. We investigated splits identified based on the divergence time estimation and on the ancestral range reconstruction analyses that were relevant to our hypotheses at all levels in the phylogeny.

An ancestral range reconstruction was performed in BioGeoBEARS where a dispersal-extinction-cladogenesis (DEC) model was found as the best-fit model (DEC AIC: 1406, DIVALIKE AIC: 1585, BAYAREALIKE AIC: 1734). The ancestral area for Sapotaceae was reconstructed as Asia (Fig. 4a Node a). Our results suggest that Sapotaceae arrived first to the Amazon from the Palaeotropics and from the Amazon it migrated to other areas in South and Central America, and the West Indies. After arrival to Central America, Chrysophylloideae colonised Choco and after arrival to the Atlantic Forest it colonised the Magdalena Valley. Direct dispersal from the Amazon was reconstructed for the Guianas and Macarena, and this was followed by multiple dispersals between South and Central America, and the West Indies (Table 1 and Fig. 4).

Thirty-two splits between west-Andean and east-Andean taxa were found from ca. 24 Ma onwards, of which 18 had posterior probability (pp.) values higher than 0.9 (Table 1; Fig. 4a-c). Fifteen splits among lineages in Central America and South America were found from ca. 33 Ma, nine of them with pp. $>0.9$ (Fig. 4a). Lastly, ten splits between a

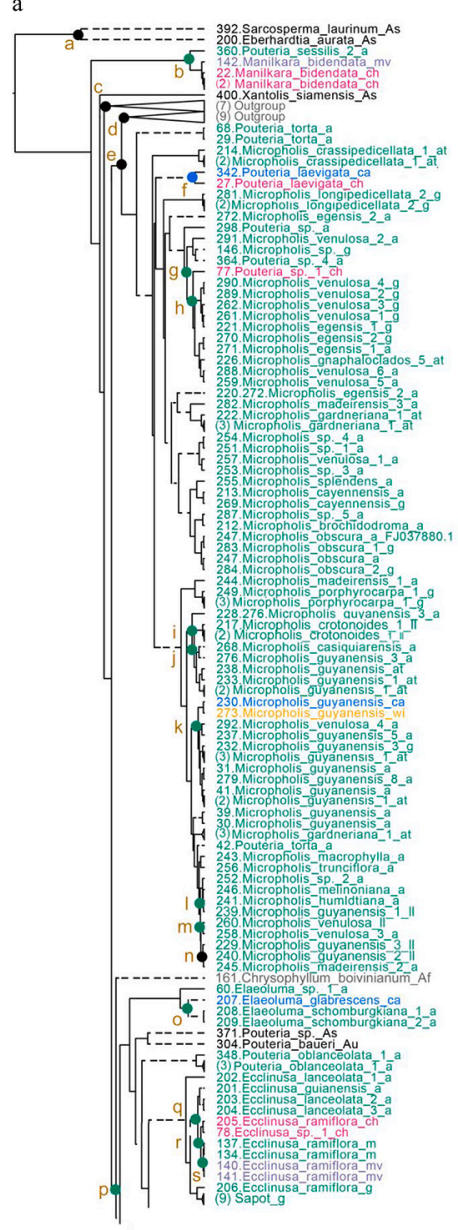

b

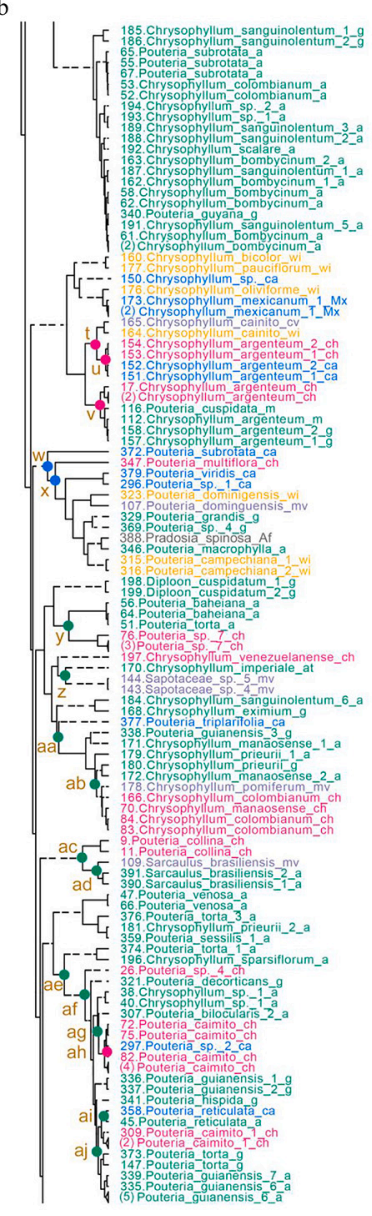

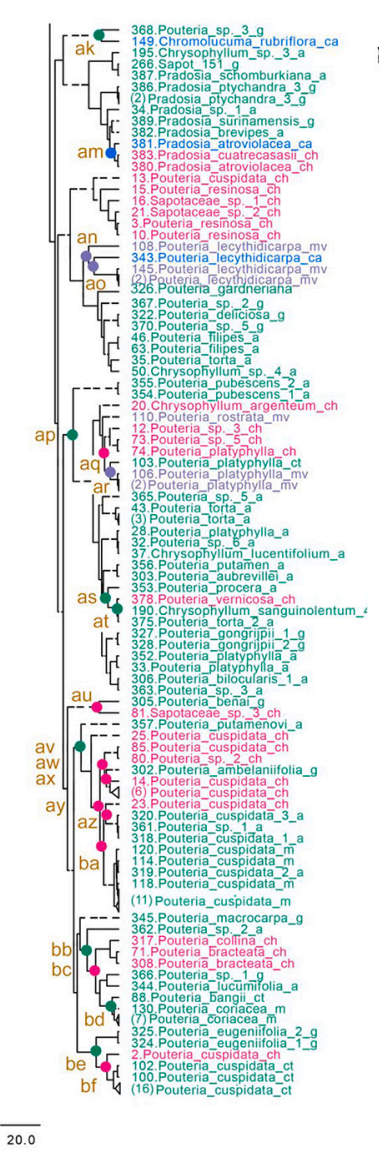

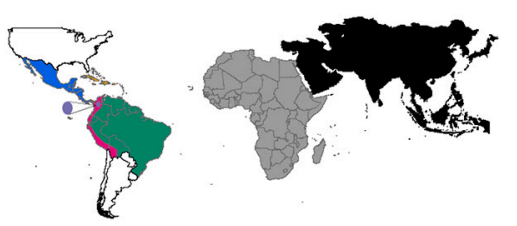

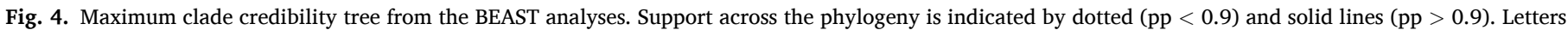

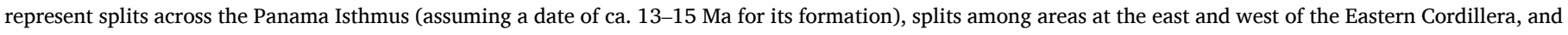

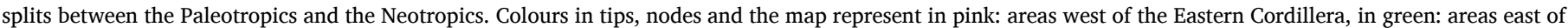

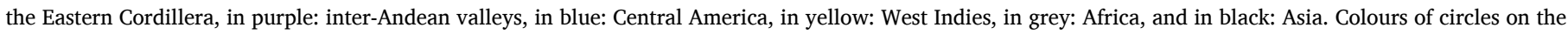

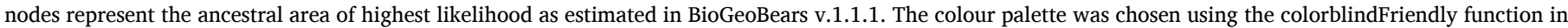

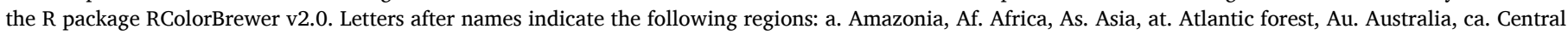

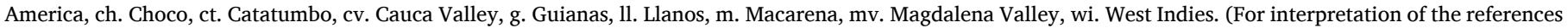
to colour in this figure legend, the reader is referred to the web version of this article.) 
Table 1

Summary of the divergence time estimation. Divergence time estimation analyses were carried out using BEAST v1.8.4. HPD are given for those nodes with Posterior Probability values higher than 0.7. Splits were divided into: TransAndean (between Chocó/Cauca valley/Magdalena Valley and Amazon/ Atlantic forest/ Macarena/Catatumbo); east-Andean (between Amazon, Catatumbo, and Macarena); Across the Panama Isthmus (between Central America and South American areas); trans-Atlantic (between Paleotropics and Neotropics); and Paleotropical for areas in Africa and Asia.

\begin{tabular}{|c|c|c|c|c|}
\hline Node & $\begin{array}{l}\text { Posterior } \\
\text { probability }\end{array}$ & $\begin{array}{l}\text { Mean age and } \\
\text { HPD (Mya) }\end{array}$ & Type of split & $\begin{array}{l}\text { Ancestral Area } \\
\text { (highest likelihood) }\end{array}$ \\
\hline $\mathrm{a}$ & 0.83 & $\begin{array}{l}65.66[35.22, \\
94.92]\end{array}$ & Trans-Atlantic & Asia \\
\hline aа & 0.19 & 27.1 & $\begin{array}{l}\text { Panama } \\
\text { Isthmus }\end{array}$ & Amazon \\
\hline$a b$ & 0.32 & 6.93 & Trans-Andean & Amazon \\
\hline ac & 1 & $\begin{array}{l}14.3[7.47, \\
20.29]\end{array}$ & Trans-Andean & Amazon \\
\hline ad & 1 & $\begin{array}{l}6.11[2.45, \\
8.92]\end{array}$ & Trans-Andean & Amazon \\
\hline ae & 0.87 & $\begin{array}{l}24[15.9, \\
31.37]\end{array}$ & Trans-Andean & Amazon \\
\hline af & 0.86 & $\begin{array}{l}13.01 \text { [6.99, } \\
19.49]\end{array}$ & $\begin{array}{l}\text { Panama } \\
\text { Isthmus }\end{array}$ & Amazon \\
\hline ag & 0.25 & 5.8 & Trans-Andean & Amazon \\
\hline $\mathrm{ah}$ & 0.2 & 1.1 & $\begin{array}{l}\text { Panama } \\
\text { Isthmus }\end{array}$ & Chocó \\
\hline ai & 0.23 & 1.6 & $\begin{array}{l}\text { Panama } \\
\text { Isthmus }\end{array}$ & Amazon \\
\hline aj & 0.39 & 6 & Trans-Andean & Guianas \\
\hline ak & 1 & $\begin{array}{l}9.4[3.66, \\
14.79]\end{array}$ & $\begin{array}{l}\text { Panama } \\
\text { Isthmus }\end{array}$ & Guianas \\
\hline am & 1 & $\begin{array}{l}2.7[0.69, \\
3.70]\end{array}$ & $\begin{array}{l}\text { Panama } \\
\text { Isthmus }\end{array}$ & Central America \\
\hline an & 0.95 & $\begin{array}{l}15.9 \text { [9.64, } \\
21.13]\end{array}$ & $\begin{array}{l}\text { Panama } \\
\text { Isthmus }\end{array}$ & Magdalena valley \\
\hline ao & 0.92 & $\begin{array}{l}13.1[7.24, \\
18.06]\end{array}$ & $\begin{array}{l}\text { Panama } \\
\text { Isthmus }\end{array}$ & Magdalena valley \\
\hline ap & 0.89 & $\begin{array}{l}23.7[15.95, \\
30.56]\end{array}$ & Trans-Andean & Amazon \\
\hline aq & 0.3 & 7.8 & Trans-Andean & Chocó \\
\hline ar & 1 & $\begin{array}{l}4.96[1.90, \\
6.78]\end{array}$ & Trans-Andean & Magdalena valley \\
\hline as & 0.99 & $6[2.74,8.41]$ & Trans-Andean & Amazon \\
\hline at & 1 & $\begin{array}{l}1.4[0.06 \\
1.76]\end{array}$ & Trans-Andean & Amazon \\
\hline au & 1 & $\begin{array}{l}10.65 \text { [4.5, } \\
16.12]\end{array}$ & $\begin{array}{l}\text { Panama } \\
\text { Isthmus }\end{array}$ & Chocó \\
\hline av & 0.15 & 19.79 & Trans-Andean & Amazon \\
\hline aw & 0.71 & $\begin{array}{l}7.84[4.18, \\
10.31]\end{array}$ & Trans-Andean & Chocó \\
\hline $\mathrm{ax}$ & 0.39 & 6.94 & Trans-Andean & Chocó \\
\hline ay & 0.04 & 7.2 & Trans-Andean & Chocó \\
\hline $\mathrm{az}$ & 0.35 & 10.16 & Trans-Andean & Chocó \\
\hline $\mathrm{b}$ & 1 & $\begin{array}{l}8.1[2.89, \\
12.96]\end{array}$ & Trans-Andean & Amazon \\
\hline ba & 0.05 & 8.2 & Trans-Andean & Chocó \\
\hline $\mathrm{bb}$ & 1 & $\begin{array}{l}16[11.09, \\
20.74]\end{array}$ & Trans-Andean & Amazon \\
\hline bc & 0.99 & $\begin{array}{l}12.3[7.74, \\
15.61]\end{array}$ & Trans-Andean & Chocó \\
\hline bd & 1 & $\begin{array}{l}3.9[1.33, \\
5.48]\end{array}$ & Trans-Andean & Catatumbo \\
\hline be & 0.96 & $\begin{array}{l}12.1[6.4, \\
17.07]\end{array}$ & Trans-Andean & Guyanas \\
\hline bf & 0.98 & $\begin{array}{l}6.5 \text { [2.83, } \\
9.43]\end{array}$ & Trans-Andean & Chocó \\
\hline c & 0.34 & 50 & Paleotropical & Africa \\
\hline d & 0.88 & $\begin{array}{l}40[35.08, \\
46.19]\end{array}$ & Paleotropical & Africa \\
\hline e & 0.17 & 44 & Trans-Atlantic & Africa \\
\hline $\mathrm{f}$ & 0.99 & $\begin{array}{l}5.6[1.11, \\
9.76]\end{array}$ & $\begin{array}{l}\text { Panama } \\
\text { Isthmus }\end{array}$ & Central America \\
\hline$g$ & 0.41 & 9.74 & Trans-Andean & Amazon \\
\hline $\mathrm{h}$ & 0.98 & $\begin{array}{l}6.57[3.24, \\
8.07]\end{array}$ & Trans-Andean & Amazon \\
\hline $\mathrm{i}$ & 0.96 & $7[3.95,9.68]$ & East-Andean & Amazon \\
\hline
\end{tabular}

Table 1 (continued)

\begin{tabular}{|c|c|c|c|c|}
\hline Node & $\begin{array}{l}\text { Posterior } \\
\text { probability }\end{array}$ & $\begin{array}{l}\text { Mean age and } \\
\text { HPD (Mya) }\end{array}$ & Type of split & $\begin{array}{l}\text { Ancestral Area } \\
\text { (highest likelihood) }\end{array}$ \\
\hline j & 0.12 & 5.9 & East-Andean & Amazon \\
\hline $\mathrm{k}$ & 0.03 & 3.8 & $\begin{array}{l}\text { Panama } \\
\text { Isthmus }\end{array}$ & Amazon \\
\hline 1 & 0.11 & 1 & East-Andean & Amazon \\
\hline $\mathrm{m}$ & 0.95 & $\begin{array}{l}1.2[0.01, \\
1.49]\end{array}$ & East-Andean & Amazon \\
\hline $\mathrm{n}$ & 0.1 & 1 & East-Andean & Llanos \\
\hline o & 0.48 & 9.09 & $\begin{array}{l}\text { Panama } \\
\text { Isthmus }\end{array}$ & Amazon \\
\hline $\mathrm{p}$ & 0.13 & 47 & Trans-Atlantic & Africa \\
\hline $\mathrm{q}$ & 1 & $4[1.49,5.47]$ & Trans-Andean & Amazon \\
\hline $\mathrm{r}$ & 0.95 & $\begin{array}{l}2.4[0.55, \\
3.17]\end{array}$ & Trans-Andean & Amazon \\
\hline $\mathrm{s}$ & 0.99 & $\begin{array}{l}1.5[0.05, \\
1.11]\end{array}$ & Trans-Andean & Macarena \\
\hline $\mathrm{t}$ & 1 & $\begin{array}{l}6.8[2.84, \\
9.64]\end{array}$ & Trans-Andean & Chocó \\
\hline $\mathrm{u}$ & 1 & $2[0.23,2.75]$ & $\begin{array}{l}\text { Panama } \\
\text { Isthmus }\end{array}$ & Chocó \\
\hline $\mathrm{v}$ & 1 & $4.8[1.79,7]$ & Trans-Andean & Chocó \\
\hline $\mathrm{w}$ & 0.99 & $\begin{array}{l}33[24.18, \\
40.64]\end{array}$ & $\begin{array}{l}\text { Panama } \\
\text { Isthmus }\end{array}$ & Central America \\
\hline $\mathrm{x}$ & 0.96 & $\begin{array}{l}28[20.68, \\
36.33]\end{array}$ & $\begin{array}{l}\text { Panama } \\
\text { Isthmus }\end{array}$ & Central America \\
\hline $\mathrm{y}$ & 0.99 & $\begin{array}{l}21.6[12.62, \\
29.78]\end{array}$ & Trans-Andean & Amazon \\
\hline $\mathrm{z}$ & 0.73 & $\begin{array}{l}23[12.68, \\
34.53]\end{array}$ & Trans-Andean & Atlantic forest \\
\hline
\end{tabular}

neotropical Sapotaceae species occurred during the Pleistocene epoch, seven of them with pp. $>0.9$.

We found two major neotropical clades derived from African lineages at ca. 44 Ma (Fig. 4a Node e, pp. 0.17) and ca. 47 Ma (Node p. Fig. 4a, pp. 0.13), respectively. Additionally, a well-supported neotropical clade nested within the Asian Sapotaceae was found and includes taxa from the Amazon, Magdalena Valley and Chocó (ca. 8.1 [2.89-12.96 HPD] Ma, Fig. 4a Node b, pp. 1).

The earliest well-supported split between Chocó and any other area in the Neotropics occurred at ca. 33 (24.18-40.64 HPD) Ma (Fig. 4b Node w, pp. 0.99), between Chocó and Central America (pp. 0.99). Further splits between Chocó and the Guyanas occurred between ca. 13 and 6.5 Ma (Table 1). Well supported splits between Chocó and Central America occurred at $28 \mathrm{Ma}(20.68-36.33 \mathrm{HPD}$; Fig. 4a Node x, pp. 0.96), and more recently at ca. 5.6 Ma (1.11-9.76 HPD; Fig. 4a Node f, pp. 0.99), 2.7 (0.69-3.70 HPD) Ma (0.69-3.70 HPD; Fig. 4c Node am, pp. 1), and 2 (0.23, 2.75 HPD) Ma (Fig. 4b Node u, pp. 1).

Three well supported splits between Central America and other areas in South America occurred at ca. 15.9 (9.64-21.13 HPD), 13 (7.24-18.06 HPD) and 9.4 (3.66-14.79 HPD) Ma (Fig. 4c Nodes an, ao, ak). Six additional splits among lineages in Central America and South America were found from 27.1 Ma to $1.6 \mathrm{Ma}$, but without strong statistical support (Fig. 4a,b, Nodes aa, o, ai, k, aj).

The oldest split between lineages from Chocó and the Amazon occurred at ca. 24 (15.9-31.37 HPD) Ma (Fig. 4b Node ae, pp. 0.87). However, this and a later split at ca. 23.7 (15.95-30.56 HPD) Ma (Fig. 4b Node ap) are weakly supported (pp. 0.87 and 0.89 , respectively). Node y (Fig. 4b) is the earliest well supported (pp. 0.99) split between lineages from Chocó and the Amazon at ca. 21.6 (12.62-29.78 HPD) Ma. Six additional well-supported splits between taxa in Chocó and the Amazon were found between ca. 16 and 1.4 Ma (Table 1). Eight splits with pp. < 0.9 occurred between taxa in the Amazon and Chocó from ca. 19.7 (35.08-46.19 HPD) Ma to ca. 5.1 Ma (Fig. 4a,b,c Nodes av., az, g, ab, ag).

The oldest split between Chocó and other areas in northern South America such as the Cauca valley was found at ca. 6.8 (2.84-9.64 HPD) Ma (Fig. 4b Node t, pp. 1), and between Chocó and Magdalena Valley at ca. 14.3 (7.47-20.29 HPD) Ma (Fig. 4b Node ac, pp. 1). Another split 
between Chocó and Magdalena Valley occurred at ca. 2.4 (0.55-3.17 HPD) Ma (Fig. 4a Node r, pp. 0.95).

Node z (Fig. 4b) at ca. 23 (12.68-34.53 HPD) Ma (pp. 0.73) indicates the time when lineages in the Magdalena valley and the Atlantic forest in Brazil last shared a common ancestor. The earliest split between taxa in the Magdalena valley and the Amazon occurred at 8.1 (2.89-12.96 HPD) Ma (Fig. 4a Node b, pp. 1), taxa shared between these areas also diverged later at ca. 6.1 Ma (2.45-8.92 HPD; Fig. 4b Node ad, pp. 1). The age of node s (Fig. 4a) at ca. 1.5 (0.05-1.11 HPD) Ma (pp. 0.99) represents a younger split between lineages from the Magdalena valley and Macarena.

Two main clades of lineages from Macarena are represented by nodes ba and bd (Fig. 4c). Node ba represents the most recent common ancestor shared between lineages from Macarena and Chocó/Amazon at ca. 8.2 Ma. This relationship is weakly supported (pp. 0.05). Node bd indicates the most recent common ancestor shared between taxa in Catatumbo and in Macarena at ca. 3.9 (1.33-5.48 HPD) Ma (pp. 1). Taxa in Macarena were found to also share a common ancestor with taxa in the Amazon at ca. $4 \mathrm{Ma}$ (pp.1) and in Chocó at ca. 4.8 (1.79-7 HPD) Ma (Fig. 4d Node V, pp. 1) and 2.4 (0.55, 3.17) Ma (Fig. 4a Node r, pp. 0.95).

Catatumbo lineages (Fig. 4c Node be) diverged from lineages from Chocó at ca. 6.5 (2.83, 9.43 HPD) Ma (Fig. 4c Node bf, pp. 0.98). The stem node of that clade was found at ca. $12 \mathrm{Ma}$ (pp. 0.96) and represents the split between taxa in the Guyanas and clade 4.13. Taxa from Catatumbo split from taxa in the Magdalena valley at ca. 4.9 (1.90, 6.78 HPD) and 7.8 (1.90-6.78 HPD) Ma (Fig. 4c Nodes ar and aq, pp. 1 and $0.3)$. Lineages from Los Llanos split from taxa from the Amazon at ca. 1 Ma (Fig. 4a Node n, pp. 0.1 and Node 1, pp. 0.11), 1.2 (0.01, 1.49 HPD) Ma (Fig. 4a Node m. pp. 0.95), 5.9 Ma (Node j. pp. 0.12) and 7 (3.95, 9.68 HPD) Ma (Node i. pp. 0.96).

\subsection{Diversification rates}

In our phylogeny the probability of a null model was too low and it could not be calculated by BAMM. However, BAMM sampled models with 1:5 and 7 shift regimes, favouring a model with a single shift at the basal node of the Neotropical groups (Fig. 3). Branch specific speciation rates calculated by RevBayes recovered two additional shifts in diversification during the late Miocene at ca. 6-8 Ma (Fig. 3), for lineages in the Catatumbo and Macarena forests. Changes in diversification rates coinciding with periods of major uplift of the Andes, the closure of the Isthmus of Panama, or Pleistocene climatic changes were not supported (Fig. 3).

\section{Discussion}

The uplift of the Andes, the formation of the Panama land bridge and the climatic changes in the Pleistocene reconfigured the Neotropics generating barriers that could assist and prevent dispersal and promote diversification. In our analyses we did not, however, find evidence supporting the hypotheses of increases in diversification rates caused by the Andean uplift or the Pleistocene climatic changes. Our data suggest that dispersal has played an important role in the evolutionary history of neotropical Chrysophylloideae at various scales, with deep signatures of its effects in our phylogeny from the arrival of the subfamily to South America possibly via trans-oceanic dispersal, to migration events across the Andes and the Panama land bridge, to fine patterns of evolution within and between species across Sapotaceae's geographical range.

The earliest record for a possible Sapotaceae migration from Asia to the Neotropics was found in a well-supported neotropical clade nested within the Asian species with a stem node age of ca. 60 (56-67 HDP) Ma (Fig. 4a Node b). We found evidence for further and later migration to the Neotropics from Africa at ca. 47 and $44 \mathrm{Ma}$. Arrival in the Neotropics was accompanied by a significant shift in diversification rates (Fig. 3), although this may be an artefact of low sampling of palaeotropical lineages. These dispersal events postdate the physical isolation of South
America from Africa, and coincided with the existence of the megathermal boreotropical rain forest in the northern hemisphere during the Paleocene/Eocene (Wolfe, 1975). However, one would expect South American lineages to be nested within Central American ones if South America had been colonised from northern boreotropical regions. According to the ancestral reconstruction in BioGeoBEARS, the earliest occurrence of Sapotaceae in the Neotropics was in the region currently occupied by the Amazon basin (Fig. 4 and Table 1). Hence, Sapotaceae could have dispersed instead via oceanic currents from Africa to the eastern coast of South America as previously suggested for the Sapotaceae genus Manilkara (Armstrong et al., 2014) (Table 1). Chrysophylloideae then migrated on multiple occasions to Central America, to the West Indies, and other areas in South America.

Evidence suggesting a strong role of dispersal in Chrysophylloideae's history was also found at finer scales in our phylogeny where we identified non-monophyletic species with wider distributions, which may be the ancestors of species with narrower ranges that are phylogenetically nested within them. This pattern deserves further study and was found in species such as Chrysophyllum cainito that is nested within C. argenteum, Micropholis casiquariensis that is nested within $M$. guyanensis, and Pouteria reticulata and $P$. caimito that are nested within the more broadly distributed $P$. guianensis. Wide distributional ranges in lowland rain forest could be the result of dispersal events followed by successful colonisation in areas where empty spaces were created after periods of frequent disturbance (Pennington and Lavin, 2016). Isolation and drift or strong selection may be sufficient to lead to species formation in peripheral populations.

\subsection{Trans-Andean Vicariance (Hypothesis i)}

Thirty-two splits were found between taxa at either side of the Eastern Cordillera from ca. 24 (15.9-31.37 HPD) Ma onwards (Figs. 4 and 5, and Table 1). Twenty-six of those (14 with pp. $>0.9$ and 14 with pp. < 0.9) occurred from ca. 12 Ma onwards (Table 1). We acknowledge that the total number of splits will vary if additional collections were added, yet our phylogeny includes $30 \%$ of the currently recognised species in neotropical Chrysophylloideae, including $71 \%$ of the known species in Colombia, one of the most species-rich areas and where the Andes branches in three ranges separating important fragments of lowland rain forest. Our taxon sampling is adequate to recover important evidence for migration across mountain barriers and reject a hypothesis of vicariance caused by the uplift of the Eastern Cordillera (Fig. 2). Under this vicariance hypothesis we would expect splits on either side to have occurred mainly from $c a$. $12 \mathrm{Ma}$. Timing of isolation across the Cordillera in our analyses is spread through time rather than concentrated at one point as a vicariance model would predict (Fig. 5). This indicates that the Andes were not a significant barrier to dispersal, and that isolation of Sapotaceae lineages on either side of the Andes's Eastern Cordillera after the middle Miocene, the time frame of major uplift in northern South America, occurred only in some cases. Frequent dispersal among areas separated by the Andes has been reported in other studies by Fine et al. (2014), Dexter et al. (2017), Hazzi et al. (2018), Pérez-Escobar et al. (2019), and like Serrano et al. (2018) they indicate that migration may have prevented provincialism in Sapotaceae species distributions (but see for examples where dispersal may have been less prevalent Trénel et al., 2007; Albert et al., 2006; Pirie et al., 2006; Arrivillaga et al., 2002; Winterton et al., 2014; De-Silva et al., 2016).

Migration in Chrysophylloideae between areas to the west of the Eastern Cordillera, i.e. Chocó, and the inter-Andean valleys, would not have been limited by altitude as these areas are all connected by fragments of lowland rain forest. These fragments are in the departments of Antioquia, Cordoba and Bolivar (Gentry, 1982) (Fig. 1). Dispersal between Chocó and other areas in northern South America has also been reported in other taxa such as Arecaceae and Burseraceae (Fine et al., 2014; Dexter et al., 2017; Bacon et al., 2018). trans-Andean migration of Sapotaceae between areas that are not connected by corridors of 


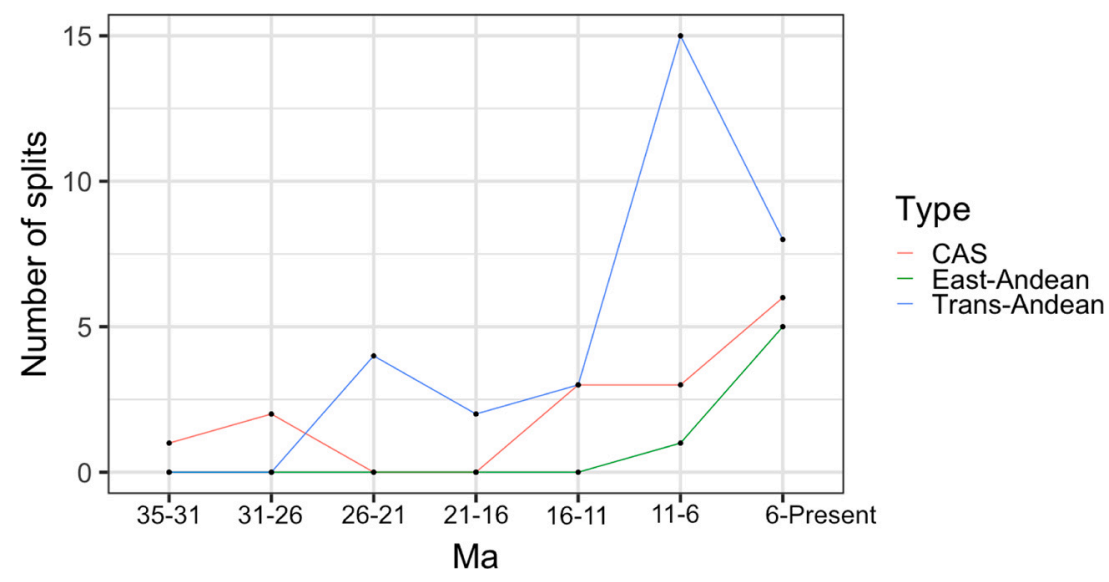

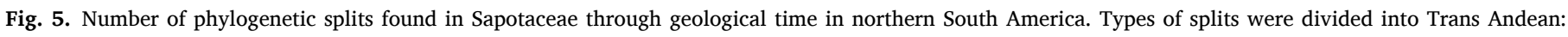

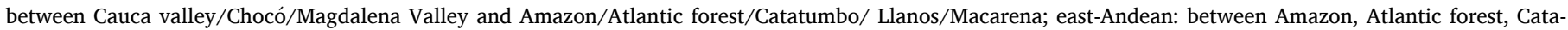
tumbo, Llanos and Macarena; and Across CAS (Central American Seaway): between Central America/Mexico and South American areas.

lowland rain forest such as Catatumbo and the inter-Andean valleys, could have occurred through mountain passes where altitudes in the Eastern Cordillera are lower (Hazzi et al., 2018; Serrano et al., 2018) (red bars Fig. 1, Fig. 4).

\subsection{Migration across the Panama Isthmus (Hypothesis ii)}

Fifteen migration events, nine of them well supported, were found between Central and South America (Figs. 4 and 5). The rise of the Isthmus of Panama was a crucial event for Neotropical biotic evolution as it connected North and South America allowing the interchange of terrestrial species (Simpson, 1980). According to Coates \& Obando (1996) the formation of the Isthmus of Panama did not occur in one single event, but was reportedly completed in the Middle Pliocene at around 3.4-3.1 Ma. However, more recent studies based on river deposit evidence from Northern South America, indicate that the land bridge may actually have been formed at around 13-15 ma. The migration events found in our phylogeny occurred from ca. 33 (24.18-40.64 HPD) Ma (Node w, Fig. 4b.) onwards, prior to and after the closure of the Panama land bridge, assuming a date of closure of ca. 13-15 Ma (Hoorn and Flantua, 2015; Bacon et al., 2015). Our results corroborate Cody et al. (2010) who compared plant and animal migration patterns across the isthmus, and found that dispersal of plant taxa was spread through the Cenozoic and often preceded the formation of the land bridge (whether regarded as occurring at $3 \mathrm{Ma}$ or 13-15 Ma). They are also similar to those of Bartish et al. (2011) who suggested that the closure of the Central American Seaway initiated a possible route for migration but was not essential for the expansion of Sapotaceae's distributional range. Considering that there is evidence for transatlantic dispersal in Sapotaceae (e.g. Bartish et al., 2011), migration could have taken place from South America to Central and North America, directly across the relatively narrow seaway present before the formation of the Isthmus of Panama.

\subsection{Diversification after the Andean uplift (Hypothesis iii)}

Sapotaceae diversified at a constant rate until about 49.5 Ma when arrival in the Neotropics coincided with shifts in diversification rates (Figs. 3 and 5). Two additional diversification shifts occurred in the late Miocene in clades representing taxa at the Macarena and Catatumbo forests. The hypothesis of increased diversification rates being associated with periods of major uplift in the Andes was not supported. Because Sapotaceae species do not generally occur above $2000 \mathrm{~m}$ in South America, so they would not have been affected by the creation of novel montane habitats by the Andean uplift. This uplift could, however, have affected the evolution of Chrysophylloideae and other lowland rain forest restricted taxa by altering the edaphic and hydrological systems in lowland areas (Burnham and Graham, 1999; Hoorn et al., 2010), but the effects of these alterations did not significantly increase diversification rates in our analyses.

\subsection{Pleistocene diversification (Hypothesis iv)}

Despite early work suggesting that speciation in animals from the lowland rain forest of South America mostly predates the Pleistocene (Moritz et al., 2000), more recently examples have accumulated suggesting origin of neotropical rain forest species during the Pleistocene including in Inga (Fabaceae), Guatteria (Annonaceae) and Meliaceae (Richardson et al., 2001; Koenen et al., 2015). Thirteen splits (seven with pp. > 0.9) among South American Sapotaceae occurred during the Pleistocene (Fig. 5). Our results add to a building picture that species diversification in neotropical trees has occurred recently, and Pleistocene dates for speciation in Sapotaceae (Fig. 5 and Table 1) are consistent in timing with a speciation model driven by climatic changes. However, Pleistocene speciation is not responsible for the majority of Sapotaceae speciation as early "refuge theory" models of neotropical speciation hypothesised (Haffer, 1969; Prance, 1973; Richardson et al., 2001; Pérez-Escobar et al., 2019).

\section{Conclusions}

The study of patterns of evolution in northern South America, is a fundamental piece to our understanding of the origins of the great plant diversity of the Neotropics. Yet, due to decades of civil conflict in countries like Colombia, the addition of plant collections from forests on both sides of the Andes, was highly restricted. Here we addressed this gap and tested hypotheses focussed on the role of geographic barriers and climatic changes in the biogeographic history of the Chrysophylloideae subfamily in Sapotaceae. Our results uncover patterns suggesting a strong role for dispersal in the history of neotropical Sapotaceae and the lowland rain forest biome, where they are most abundant.

\section{Author contributions}

Conceived and designed the experiments: JER, JS. Generated the data and ran analyses JS. Wrote the paper: JS, JER, RTP, RIM, RCB, GAM, JAH, IVB, MG, JC, SM, DC, SDC. 


\section{Declaration of Competing Interest}

The authors declare that they have no known competing financial interests or personal relationships that could have appeared to influence the work reported in this paper.

\section{Acknowledgements}

We thank Michelle Hart and Laura Forest for laboratory assistance at the Royal Botanic Garden Edinburgh. Fabio Ávila and Doris Huertas are thanked for their assistance at the Herbario Forestal. Terry Pennington and Felix Forest at the Royal Botanic Gardens, Kew are thanked for providing samples of Colombian Sapotaceae and permitting their extraction respectively. Alain Franc supplied samples from French Guiana that were collected by Daniel Sabatier and Jean-François Molino all of whom are thanked for those contributions. Investissement d'Avenir" grants managed by Agence Nationale de la Recherche (CEBA, ref. ANR-10-LABX-25-01) is thanked for their financial support to the work carried out by Jérôme Chave's Laboratory. Unpublished data was provided by Dayana Sanchez from the District University in Bogotá, Colombia. Specimen collection in Colombia was performed under the general collecting permit awarded to Universidad de los Andes and Universidad Distrital Francisco José de Caldas. The work was supported by a University of Edinburgh Darwin Trust PhD Scholarship awarded to the first author.

\section{Appendix A}

List of specimens included in the phylogenetic analyses. Internal transcribed spacers of rDNA (ITS) sequence data was generated. A total of 146 Chrysophylloideae accessions were collected in the Choco, Magdalena valley, Catatumbo, Amazon and Macarena lowland rain forests of Colombia. The remaining sequences were obtained from previous phylogenetic studies on Chrysophylloideae (Sánchez-C. et al., 2021; Gonzalez et al., 2009; Swenson et al., 2008) and GenBank

*sterile specimens not deposited at official herbarium collections.

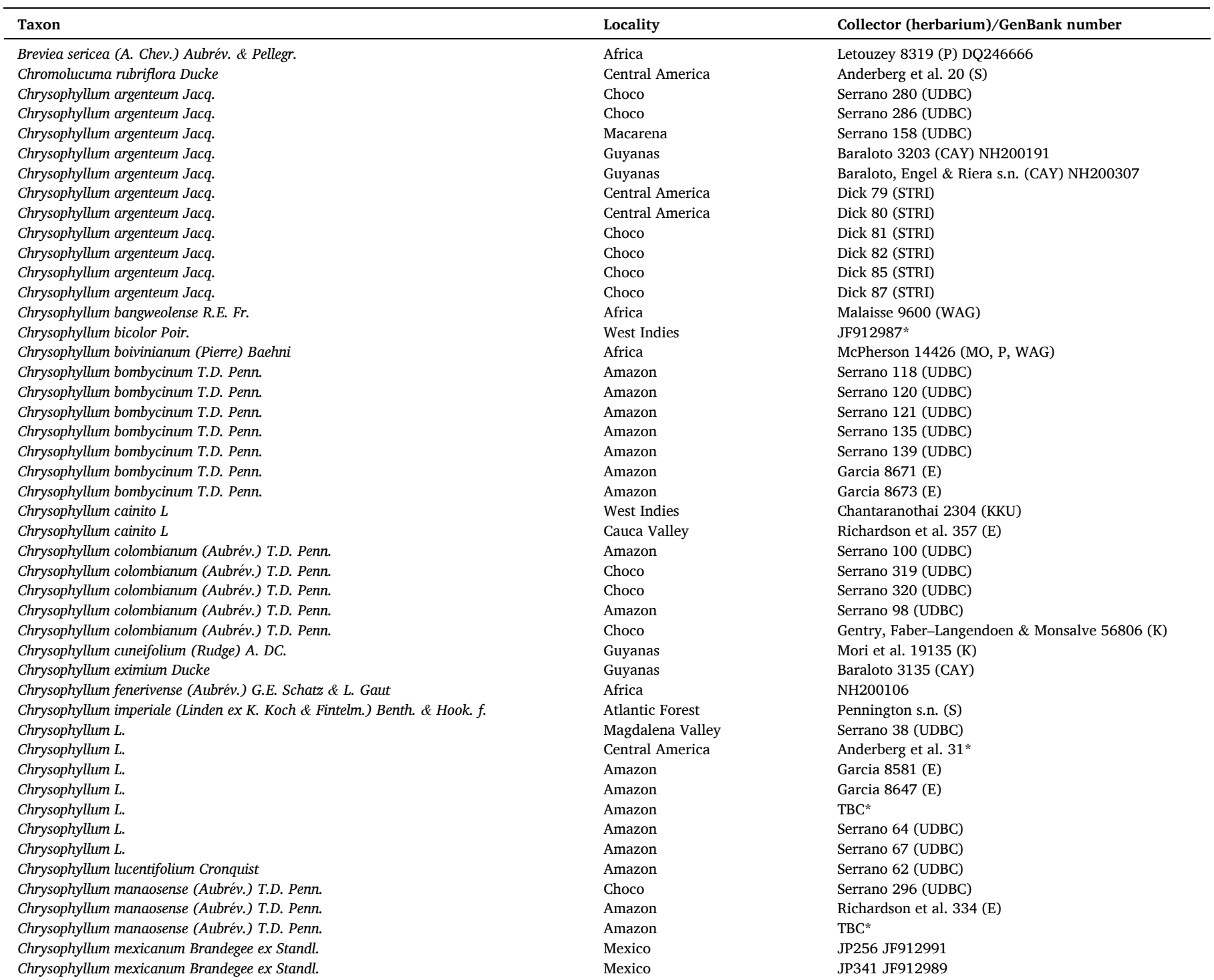




\begin{tabular}{|c|c|c|}
\hline Taxon & Locality & Collector (herbarium)/GenBank number \\
\hline Chrysophyllum mexicanum Brandegee ex Standl. & Mexico & JP398 JF912990 \\
\hline Chrysophyllum oliviforme $L$. & West Indies & Guttiérrez \& Nilsson 1 (S) \\
\hline Chrysophyllum pauciflorum Lam & West Indies & JF912986 \\
\hline Chrysophyllum pomiferum (Eyma) T.D. Penn. & Magdalena Valley & Pennington et al. $12386^{*}$ \\
\hline Chrysophyllum prieurii A. DC. & Guyanas & Baraloto, Engler \& Chave s.n. (CAY) P01860227 \\
\hline Chrysophyllum prieurii A. DC. & Amazon & Garcia 8583 (E) \\
\hline Chrysophyllum prieurii $A . D C$. & Amazon & Richardson et al. 326 (E) \\
\hline Chrysophyllum pruniforme Pierre ex Engl. & Africa & Jongkind 3762 (WAG) \\
\hline Chrysophyllum roxburghii G. Don & Africa & Randrianasolo 33 (WAG) \\
\hline Chrysophyllum sanguinolentum (Pierre) Baehni & Guyanas & Baraloto 3016 (CAY) \\
\hline Chrysophyllum sanguinolentum (Pierre) Baehni & Guyanas & Baraloto 3196 (CAY) NH200177 \\
\hline Chrysophyllum sanguinolentum (Pierre) Baehni & Amazon & FJ037869 \\
\hline Chrysophyllum sanguinolentum (Pierre) Baehni & Amazon & Garcia $8236(E)$ \\
\hline Chrysophyllum sanguinolentum (Pierre) Baehni & Amazon & Garcia 8645 (E) \\
\hline Chrysophyllum sanguinolentum (Pierre) Baehni & Amazon & Richardson et al. 305 (E) \\
\hline Chrysophyllum sanguinolentum (Pierre) Baehni & Amazon & Richardson et al. 310 (E) \\
\hline Chrysophyllum sanguinolentum (Pierre) Baehni & Amazon & Richardson et al. 348 (E) \\
\hline Chrysophyllum scalare T.D. Penn. & Amazon & Richardson et al. 320 (E) \\
\hline Chrysophyllum sparsiflorum Klotzsch ex Miq. & Amazon & DQ021882 \\
\hline Chrysophyllum venezuelanense (Pierre) T.D. Penn & Choco & Ståhl et al. 5755 (S) \\
\hline Diploon cuspidatum (Hoehne) Cronquist & Guyanas & Baraloto 3209 (CAY) NL110264 \\
\hline Diploon cuspidatum (Hoehne) Cronquist & Guyanas & Pennington et al. 13843 (U) \\
\hline Eberhardtia aurata (Pierre ex Dubard) Lecomte & Asia & Hao 534 (S) EF558617 \\
\hline Ecclinusa guianensis Eyma & Amazon & Ducke Res. 05-906 (K) \\
\hline Ecclinusa lanceolata (Mart. \& Eichler) Pierre & Amazon & Garcia $8675(\mathrm{E})$ \\
\hline Ecclinusa lanceolata (Mart. \& Eichler) Pierre & Amazon & Garcia 8689 (E) \\
\hline Ecclinusa lanceolata (Mart. \& Eichler) Pierre & Amazon & Richardson et al. 314 (E) \\
\hline Ecclinusa Mart. & Choco & Serrano 309 (UDBC) \\
\hline Ecclinusa ramiflora Mart. & Macarena & Serrano 170 (UDBC) \\
\hline Ecclinusa ramiflora Mart. & Macarena & Serrano 177 (UDBC) \\
\hline Ecclinusa ramiflora Mart. & Guyanas & Irwing et al. 55081 (S) \\
\hline Ecclinusa ramiflora Mart. & Choco & Monsalve B1319 (K) \\
\hline Elaeoluma Baill. & Amazon & Serrano 131 (UDBC) \\
\hline Elaeoluma glabrescens (Mart. \& Eichler) Aubrév. & Central America & Anderberg et al. 33 (S) \\
\hline Elaeoluma schomburgkiana (Miq.) Baill. & Amazon & DQ246679 \\
\hline Elaeoluma schomburgkiana (Miq.) Baill. & Amazon & Keel \& Koelho 243 (S) \\
\hline Englerophytum magalismontanum (Sond.) T.D. Penn. & Africa & Swenson \& Karis 631 (S) DQ246680 \\
\hline Englerophytum natalense (Sond.) T.D. Penn. & Africa & Kayombo 3483 (S) AY552150 \\
\hline Manilkara bidentata (A. DC.) A. Chev & Choco & Serrano 245 (UDBC) \\
\hline Manilkara bidentata (A. DC.) A. Chev & Magdalena Valley & Serrano 28 (UDBC) \\
\hline Manilkara bidentata (A. DC.) A. Chev & Choco & Serrano 294 (UDBC) \\
\hline Manilkara bidentata (A. DC.) A. Chev & Choco & Serrano 334 (UDBC) \\
\hline Micropholis (Griseb.) Pierre & Amazon & Serrano 89 (UDBC) \\
\hline Micropholis (Griseb.) Pierre & Amazon & Alvarez $737 *$ \\
\hline Micropholis (Griseb.) Pierre & Amazon & Alvarez 799 * \\
\hline Micropholis (Griseb.) Pierre & Amazon & Avila et al. 2733 (UDBC) \\
\hline Micropholis (Griseb.) Pierre & Amazon & Garcia $8618(\mathrm{E})$ \\
\hline Micropholis (Griseb.) Pierre & Amazon & Sanchez et al. $22 *$ \\
\hline Micropholis brochidodroma T.D. Penn. & Amazon & JR-JH-1* \\
\hline Micropholis casiquiarensis Aubrév. & Amazon & Richardson et al. 340 (E) \\
\hline Micropholis cayennensis T.D. Penn & Guyanas & Baraloto, Engel \& Riera s.n. (CAY) NH200222 \\
\hline Micropholis cayennensis T.D. Penn & Amazon & FJ037875.1 \\
\hline Micropholis crassipedicellata (Mart. \& Eichler ex Miq.) Pierre & Atlantic Forest & JQ434164.1 \\
\hline Micropholis crassipedicellata (Mart. \& Eichler ex Miq.) Pierre & Atlantic Forest & JQ434165.1 \\
\hline Micropholis crassipedicellata (Mart. \& Eichler ex Miq.) Pierre & Atlantic Forest & NH200222 \\
\hline Micropholis crotonoides (Pierre) Pierre & Llanos & ESR89* \\
\hline Micropholis crotonoides (Pierre) Pierre & Llanos & JRM24* \\
\hline Micropholis crotonoides (Pierre) Pierre & Llanos & Ramirez et al. 50 * \\
\hline Micropholis egensis (A. DC.) Pierre & Amazon & Avila $2746^{*}$ \\
\hline Micropholis egensis (A. DC.) Pierre & Amazon & Betancur, Echeverry, Kress \& Roesel 2848 (K) \\
\hline Micropholis egensis (A. DC.) Pierre & Amazon & Dionizia, Coêlho \& Ernesto 73 (U) \\
\hline Micropholis egensis (A. DC.) Pierre & Guyanas & DQ246681 \\
\hline Micropholis egensis (A. DC.) Pierre & Guyanas & FJ037876.1 \\
\hline Micropholis gardneriana (A. DC.) Pierre & Atlantic Forest & JQ434158.1 \\
\hline Micropholis gardneriana (A. DC.) Pierre & Atlantic Forest & JQ434158.2 \\
\hline Micropholis gardneriana (A. DC.) Pierre & Atlantic Forest & JQ434159.1 \\
\hline Micropholis gardneriana (A. DC.) Pierre & Atlantic Forest & JQ434160.1 \\
\hline Micropholis gardneriana (A. DC.) Pierre & Atlantic Forest & JQ434161.1 \\
\hline Micropholis guyanensis (A. DC.) Pierre & Amazon & Serrano 52 (UDBC) \\
\hline Micropholis guyanensis (A. DC.) Pierre & Amazon & Serrano 53 (UDBC) \\
\hline Micropholis guyanensis (A. DC.) Pierre & Amazon & Serrano 66 (UDBC) \\
\hline Micropholis guyanensis (A. DC.) Pierre & Amazon & Serrano 68 (UDBC) \\
\hline Micropholis guyanensis (A. DC.) Pierre & Amazon & Serrano 71 (UDBC) \\
\hline Micropholis guyanensis (A. DC.) Pierre & Amazon & Serrano 77 (UDBC) \\
\hline Micropholis guyanensis (A. DC.) Pierre & Amazon & Avila 2684 (UDBC) \\
\hline Micropholis guyanensis (A. DC.) Pierre & Guyanas & Baraloto 3107 (CAY) NL110516 \\
\hline
\end{tabular}




\begin{tabular}{|c|c|c|}
\hline Taxon & Locality & Collector (herbarium)/GenBank number \\
\hline Micropholis guyanensis (A. DC.) Pierre & Guyanas & Baraloto, Engel \& Riviera s.n. (CAY) NH200477 \\
\hline Micropholis guyanensis (A. DC.) Pierre & Central America & DQ246682.1 \\
\hline Micropholis guyanensis (A. DC.) Pierre & Guyanas & FJ037878.1 \\
\hline Micropholis guyanensis (A. DC.) Pierre & Amazon & Garcia $8242(\mathrm{E})$ \\
\hline Micropholis guyanensis (A. DC.) Pierre & Amazon & Garcia $8620(\mathrm{E})$ \\
\hline Micropholis guyanensis (A. DC.) Pierre & Atlantic Forest & JQ434166.1 \\
\hline Micropholis guyanensis (A. DC.) Pierre & Atlantic Forest & JQ434167.1 \\
\hline Micropholis guyanensis (A. DC.) Pierre & Atlantic Forest & JQ434168.1 \\
\hline Micropholis guyanensis (A. DC.) Pierre & Atlantic Forest & KF943856.1 \\
\hline Micropholis guyanensis (A. DC.) Pierre & Llanos & Miranda et al. 93* \\
\hline Micropholis guyanensis (A. DC.) Pierre & Llanos & Miranda et al. 93* \\
\hline Micropholis guyanensis (A. DC.) Pierre & Amazon & Piñeros et al. 14 (UDBC) \\
\hline Micropholis guyanensis (A. DC.) Pierre & Amazon & Richardson n.d * \\
\hline Micropholis guyanensis (A. DC.) Pierre & Amazon & Richardson et al. 328 (E) \\
\hline Micropholis guyanensis (A. DC.) Pierre & West Indies & Taylor 11691 (MO) \\
\hline Micropholis guyanensis (A. DC.) Pierre & Llanos & CR194* \\
\hline Micropholis guyanensis (A. DC.) Pierre & Amazon & JR-PA-08* \\
\hline Micropholis guyanensis (A. DC.) Pierre & Amazon & $\mathrm{TBC}^{*}$ \\
\hline Micropholis guyanensis (A. DC.) Pierre & Guyanas & $\mathrm{TBC}^{*}$ \\
\hline Micropholis humboldtiana (Roem. \& Schult.) T.D. Penn. & Amazon & PU72 (COAH) \\
\hline Micropholis longipedicellata Aubrév. & Guyanas & Baraloto 3210 (CAY) FJ037879 \\
\hline Micropholis longipedicellata Aubrév. & Guyanas & Baraloto 3210 (CAY) NL110459 \\
\hline Micropholis longipedicellata Aubrév. & Atlantic Forest & FJ037879.1 \\
\hline Micropholis macrophylla (Krause) T.D. Penn. & Amazon & EP102* \\
\hline Micropholis madeirensis (Baehni) Aubrév. & Amazon & Cortés et al. 2869 (UDBC) \\
\hline Micropholis madeirensis (Baehni) Aubrév. & Amazon & MS3660* \\
\hline Micropholis madeirensis (Baehni) Aubrév. & Amazon & Richardson et al. 329 (E) \\
\hline Micropholis melinoniana Pierre & Amazon & Alvarez 1999* \\
\hline Micropholis obscura T.D. Penn. & Guyanas & Baraloto 3217 (CAY) P00610293 \\
\hline Micropholis obscura T.D. Penn. & Guyanas & Baraloto 3218 (CAY) NH200217 \\
\hline Micropholis obscura T.D. Penn. & Guyanas & FJ037880.1 \\
\hline Micropholis obscura T.D. Penn. & Amazon & TBC* \\
\hline Micropholis porphyrocarpa (Baehni) Monach. & Guyanas & Baraloto 3219 (CAY) NH200696 \\
\hline Micropholis porphyrocarpa (Baehni) Monach. & Guyanas & Baraloto 3220 (CAY) NH200404 \\
\hline Micropholis porphyrocarpa (Baehni) Monach. & Guyanas & FJ037882.1 \\
\hline Micropholis porphyrocarpa (Baehni) Monach. & Guyanas & FJ037883.1 \\
\hline Micropholis splendens Gilly ex Aubrév. & Amazon & Avila et al. 2693 (UDBC) \\
\hline Micropholis trunciflora Ducke & Amazon & Cardenas $12266^{*}$ \\
\hline Micropholis venulosa (Mart. \& Eichler) Pierre & Amazon & AD8893 \\
\hline Micropholis venulosa (Mart. \& Eichler) Pierre & Amazon & Assunção 122 (U) \\
\hline Micropholis venulosa (Mart. \& Eichler) Pierre & Amazon & Avila et al. 2704 (UDBC) \\
\hline Micropholis venulosa (Mart. \& Eichler) Pierre & Guyanas & Baraloto 3114 (CAY) NL110297 \\
\hline Micropholis venulosa (Mart. \& Eichler) Pierre & Guyanas & Baraloto 3128 (CAY) NL110260 \\
\hline Micropholis venulosa (Mart. \& Eichler) Pierre & Amazon & DQ246683.1 \\
\hline Micropholis venulosa (Mart. \& Eichler) Pierre & Guyanas & FJ037884.1 \\
\hline Micropholis venulosa (Mart. \& Eichler) Pierre & Guyanas & FJ037885.1 \\
\hline Micropholis venulosa (Mart. \& Eichler) Pierre & Amazon & Garcia $8005(\mathrm{E})$ \\
\hline Micropholis venulosa (Mart. \& Eichler) Pierre & Amazon & Garcia $8238(\mathrm{E})$ \\
\hline Micropholis venulosa (Mart. \& Eichler) Pierre & Llanos & Sanchez et al. $124^{*}$ \\
\hline Omphalocarpum pachysteloides Mildbr. ex Hutch. \& Dalziel & Africa & Jongkind 2351 (WAG) AY552151 \\
\hline Omphalocarpum strombocarpum Y.B. Harv. \& Lovett & Africa & Frimodt-Møller et al. 538 (c) DQ246685 \\
\hline Omphalocarpum strombocarpum Y.B. Harv. \& Lovett & Africa & Frimodt-Møller et al. 538 (C) \\
\hline Pouteria adolfi-friedericii (Engl.) A. Meeuse & Africa & Friis et al. 3502 (UPS) \\
\hline Pouteria alnifolia (Baker) Roberty & Africa & Jongkind \& Noyes 1322 (MO) \\
\hline Pouteria altissima (A. Chev.) Baehni & Africa & Friis et al. 4145 (UPS) \\
\hline Pouteria ambelaniifolia (Sandwith) T.D. Penn. & Guyanas & Baraloto s.n. (CAY) NH200025 \\
\hline Pouteria Aubl. & Guyanas & $\mathrm{TBC}^{*}$ \\
\hline Pouteria Aubl. & Central America & Anderberg et al. 51 (S) \\
\hline Pouteria Aubl. & Central America & Anderberg et al. 60 (S) \\
\hline Pouteria Aubl. & Asia & Armstrong $317^{*}$ \\
\hline Pouteria Aubl. & Amazon & Garcia 8623 (E) \\
\hline Pouteria Aubl. & Amazon & Garcia 8632 (E) \\
\hline Pouteria Aubl. & Amazon & Garcia 8640 (E) \\
\hline Pouteria Aubl. & Amazon & Garcia 8641 (E) \\
\hline Pouteria Aubl. & Amazon & Garcia 8777 (E) \\
\hline Pouteria Aubl. & Guyanas & Sabatier \& Molino K383 \\
\hline Pouteria Aubl. & Guyanas & Sabatier \& Molino K989 \\
\hline Pouteria Aubl. & Guyanas & Sabatier \& Molino P213 \\
\hline Pouteria Aubl. & Guyanas & Sabatier \& Molino Q423 \\
\hline Pouteria Aubl. & Guyanas & Sabatier \& Molino V819 \\
\hline Pouteria Aubl. & & TBC* \\
\hline Pouteria Aubl. & Choco & Serrano 307 (UDBC) \\
\hline Pouteria Aubl. & Choco & Serrano 312 (UDBC) \\
\hline Pouteria Aubl. & Choco & Serrano 267 (UDBC) \\
\hline Pouteria Aubl. & Choco & Serrano 338 (UDBC) \\
\hline Pouteria Aubl. & Choco & Serrano 301 (UDBC) \\
\hline
\end{tabular}




\begin{tabular}{|c|c|c|}
\hline Taxon & Locality & Collector (herbarium)/GenBank number \\
\hline Pouteria Aubl. & Amazon & Serrano 54 (UDBC) \\
\hline Pouteria Aubl. & Choco & Serrano 244 (UDBC) \\
\hline Pouteria Aubl. & Choco & Serrano 246 (UDBC) \\
\hline Pouteria Aubl. & Choco & Serrano 247 (UDBC) \\
\hline Pouteria Aubl. & Choco & Serrano 306 (UDBC) \\
\hline Pouteria aubrevillei Bernardi & Amazon & Richardson et al. 327 (E) \\
\hline Pouteria baehniana Monach. & Amazon & Serrano 116 (UDBC) \\
\hline Pouteria baehniana Monach. & Amazon & Serrano 144 (UDBC) \\
\hline Pouteria bangii (Rusby) T.D. Penn. & Catatumbo & Serrano 198 (UDBC) \\
\hline Pouteria baueri (Montrouz.) Baehni & Australia & TBC* \\
\hline Pouteria benai (Aubrév. \& Pellegr.) T.D. Penn. & Guyanas & Mori et al. $27353(\mathrm{~K})$ \\
\hline Pouteria bilocularis (H.J.P. Winkl.) Baehni & Amazon & Garcia $8763(\mathrm{E})$ \\
\hline Pouteria bilocularis (H.J.P. Winkl.) Baehni & Amazon & Richardson et al. 345 (E) \\
\hline Pouteria bracteata T.D. Penn. & Choco & Serrano 298 (UDBC) \\
\hline Pouteria bracteata T.D. Penn. & Choco & Palacios $13699(\mathrm{~K})$ \\
\hline Pouteria caimito (Ruiz \& Pav.) Radlk. & Choco & Serrano 299 (UDBC) \\
\hline Pouteria caimito (Ruiz \& Pav.) Radlk. & Choco & Serrano 305 (UDBC) \\
\hline Pouteria caimito (Ruiz \& Pav.) Radlk. & Choco & Serrano 311 (UDBC) \\
\hline Pouteria caimito (Ruiz \& Pav.) Radlk. & Choco & Serrano 318 (UDBC) \\
\hline Pouteria caimito (Ruiz \& Pav.) Radlk. & Choco & Richardson et al. 377 (E) \\
\hline Pouteria caimito (Ruiz \& Pav.) Radlk. & Choco & Richardson et al. 386 (E) \\
\hline Pouteria caimito (Ruiz \& Pav.) Radlk. & Choco & Richardson et al. 387 (E) \\
\hline Pouteria caimito (Ruiz \& Pav.) Radlk. & Choco & Richardson et al. 388 (E) \\
\hline Pouteria caimito (Ruiz \& Pav.) Radlk. & Choco & Richardson et al. 390 (E) \\
\hline Pouteria caimito (Ruiz \& Pav.) Radlk. & Choco & Richardson et al. 391 (E) \\
\hline Pouteria campechiana (Kunth) Baehni & West Indies & Fagerlind $2754(\mathrm{~S})$ \\
\hline Pouteria campechiana (Kunth) Baehni & West Indies & Wang W00798 (HAST) \\
\hline Pouteria collina (Little) T.D. Penn. & Choco & Serrano 258 (UDBC) \\
\hline Pouteria collina (Little) T.D. Penn. & Choco & Serrano 263 (UDBC) \\
\hline Pouteria collina (Little) T.D. Penn. & Choco & Gentry, Faber-Langendoen \& Echevarria 62908 (K) \\
\hline Pouteria coriacea (Pierre) Pierre & Macarena & Serrano 164 (UDBC) \\
\hline Pouteria coriacea (Pierre) Pierre & Macarena & Serrano 165 (UDBC) \\
\hline Pouteria coriacea (Pierre) Pierre & Macarena & Serrano 165 (UDBC) \\
\hline Pouteria coriacea (Pierre) Pierre & Macarena & Serrano 168 (UDBC) \\
\hline Pouteria coriacea (Pierre) Pierre & Macarena & Serrano 168 (UDBC) \\
\hline Pouteria coriacea (Pierre) Pierre & Macarena & Serrano 169 (UDBC) \\
\hline Pouteria coriacea (Pierre) Pierre & Macarena & Serrano 173 (UDBC) \\
\hline Pouteria coriacea (Pierre) Pierre & Macarena & Serrano 174 (UDBC) \\
\hline Pouteria cuspidata (A. DC.) Baehni & Macarena & Serrano 156 (UDBC) \\
\hline Pouteria cuspidata (A. DC.) Baehni & Macarena & Serrano 157 (UDBC) \\
\hline Pouteria cuspidata (A. DC.) Baehni & Macarena & Serrano 159 (UDBC) \\
\hline Pouteria cuspidata (A. DC.) Baehni & Macarena & Serrano 159 (UDBC) \\
\hline Pouteria cuspidata (A. DC.) Baehni & Macarena & Serrano 160 (UDBC) \\
\hline Pouteria cuspidata (A. DC.) Baehni & Macarena & Serrano 160 (UDBC) \\
\hline Pouteria cuspidata (A. DC.) Baehni & Macarena & Serrano 161 (UDBC) \\
\hline Pouteria cuspidata (A. DC.) Baehni & Macarena & Serrano 161 (UDBC) \\
\hline Pouteria cuspidata (A. DC.) Baehni & Macarena & Serrano 162 (UDBC) \\
\hline Pouteria cuspidata (A. DC.) Baehni & Macarena & Serrano 162 (UDBC) \\
\hline Pouteria cuspidata (A. DC.) Baehni & Macarena & Serrano 166 (UDBC) \\
\hline Pouteria cuspidata (A. DC.) Baehni & Macarena & Serrano 167 (UDBC) \\
\hline Pouteria cuspidata (A. DC.) Baehni & Catatumbo & Serrano 185 (UDBC) \\
\hline Pouteria cuspidata (A. DC.) Baehni & Catatumbo & Serrano 193 (UDBC) \\
\hline Pouteria cuspidata (A. DC.) Baehni & Catatumbo & Serrano 203 (UDBC) \\
\hline Pouteria cuspidata (A. DC.) Baehni & Catatumbo & Serrano 204 (UDBC) \\
\hline Pouteria cuspidata (A. DC.) Baehni & Catatumbo & Serrano 205 (UDBC) \\
\hline Pouteria cuspidata (A. DC.) Baehni & Catatumbo & Serrano 207 (UDBC) \\
\hline Pouteria cuspidata (A. DC.) Baehni & Catatumbo & Serrano 208 (UDBC) \\
\hline Pouteria cuspidata (A. DC.) Baehni & Catatumbo & Serrano 209 (UDBC) \\
\hline Pouteria cuspidata (A. DC.) Baehni & Catatumbo & Serrano 210 (UDBC) \\
\hline Pouteria cuspidata (A. DC.) Baehni & Catatumbo & Serrano 211 (UDBC) \\
\hline Pouteria cuspidata (A. DC.) Baehni & Catatumbo & Serrano 212 (UDBC) \\
\hline Pouteria cuspidata (A. DC.) Baehni & Catatumbo & Serrano 215 (UDBC) \\
\hline Pouteria cuspidata (A. DC.) Baehni & Catatumbo & Serrano 218 (UDBC) \\
\hline Pouteria cuspidata (A. DC.) Baehni & Catatumbo & Serrano 219 (UDBC) \\
\hline Pouteria cuspidata (A. DC.) Baehni & Catatumbo & Serrano 219 (UDBC) \\
\hline Pouteria cuspidata (A. DC.) Baehni & Catatumbo & Serrano 221 (UDBC) \\
\hline Pouteria cuspidata (A. DC.) Baehni & Catatumbo & Serrano 224 (UDBC) \\
\hline Pouteria cuspidata (A. DC.) Baehni & Choco & Serrano 241 (UDBC) \\
\hline Pouteria cuspidata (A. DC.) Baehni & Choco & Serrano 242 (UDBC) \\
\hline Pouteria cuspidata (A. DC.) Baehni & Choco & Serrano 249 (UDBC) \\
\hline Pouteria cuspidata (A. DC.) Baehni & Choco & Serrano 268 (UDBC) \\
\hline Pouteria cuspidata (A. DC.) Baehni & Choco & Serrano 269 (UDBC) \\
\hline Pouteria cuspidata (A. DC.) Baehni & Choco & Serrano 284 (UDBC) \\
\hline Pouteria cuspidata (A. DC.) Baehni & Choco & Serrano 285 (UDBC) \\
\hline Pouteria cuspidata (A. DC.) Baehni & Choco & Serrano 321 (UDBC) \\
\hline Pouteria cuspidata (A. DC.) Baehni & Choco & Serrano 335 (UDBC) \\
\hline
\end{tabular}




\begin{tabular}{|c|c|c|}
\hline Taxon & Locality & Collector (herbarium)/GenBank number \\
\hline Pouteria cuspidata (A. DC.) Baehni & Choco & Serrano 336 (UDBC) \\
\hline Pouteria cuspidata (A. DC.) Baehni & Choco & Serrano 337 (UDBC) \\
\hline Pouteria cuspidata (A. DC.) Baehni & Amazon & Garcia $8624(\mathrm{E})$ \\
\hline Pouteria cuspidata (A. DC.) Baehni & Amazon & Garcia $8769(\mathrm{E})$ \\
\hline Pouteria cuspidata (A. DC.) Baehni & Amazon & Richardson et al. 337 (E) \\
\hline Pouteria decorticans T.D. Penn. & Guyanas & Baraloto, Engel, Chave \& Riera s.n. (CAY) NL110158 \\
\hline Pouteria deliciosa T.D. Penn. & Guyanas & Sabatier \& Molino $903^{*}$ \\
\hline Pouteria dominigensis (C.F. Gaertn.) Baehni & Magdalena Valley & Serrano 351 (UDBC) \\
\hline Pouteria dominigensis (C.F. Gaertn.) Baehni & West Indies & Gutiérrez \& Nilsson 13 (S) \\
\hline Pouteria eugeniifolia (Pierre) Baehni & Guyanas & Baraloto 3136 (CAY) NH200045 \\
\hline Pouteria eugeniifolia (Pierre) Baehni & Guyanas & Baraloto, Engel, Chave \& Riera s.n. (CAY) NH200157 \\
\hline Pouteria filipes Eyma & Amazon & Serrano 140 (UDBC) \\
\hline Pouteria filipes Eyma & Amazon & Serrano 73 (UDBC) \\
\hline Pouteria gardneriana (A. DC.) Radlk. & Argentina & Schwarz 8216 (UPS) \\
\hline Pouteria gongrijpii Eyma & Guyanas & Baraloto 3087 (CAY) NL110210 \\
\hline Pouteria gongrijpii Eyma & Guyanas & Baraloto 3098 (CAY) NL110282 \\
\hline Pouteria grandis Eyma & Guyanas & Sabatier \& Molino $995 *$ \\
\hline Pouteria guianensis Aubl. & Guyanas & Baraloto \& Engel s.n. (CAY) M17116516 \\
\hline Pouteria guianensis Aubl. & Guyanas & Baraloto, Engel, Chave \& Riera 3102 (CAY) NL110300 \\
\hline Pouteria guianensis Aubl. & Amazon & Garcia $8631(\mathrm{E})$ \\
\hline Pouteria guianensis Aubl. & Guyanas & Poncy $1745(\mathrm{P})$ \\
\hline Pouteria guianensis Aubl. & Amazon & Richardson et al. 309 (E) \\
\hline Pouteria guianensis Aubl. & Amazon & Richardson et al. 323 (E) \\
\hline Pouteria guianensis Aubl. & Amazon & Richardson et al. 332 (E) \\
\hline Pouteria guianensis Aubl. & Amazon & Richardson et al. 333 (E) \\
\hline Pouteria guianensis Aubl. & Amazon & Richardson et al. 342 (E) \\
\hline Pouteria guianensis Aubl. & Amazon & Richardson et al. 351 (E) \\
\hline Pouteria hispida Eyma & Guyanas & Mori et al. $25432(\mathrm{NY})$ \\
\hline Pouteria laevigata (Mart.) Radlk. & Choco & Serrano 339 (UDBC) \\
\hline Pouteria laevigata (Mart.) Radlk. & Central America & Anderberg et al. 49 (S) \\
\hline Pouteria lecythidicarpa P.E. Sánchez \& Poveda & Magdalena Valley & Serrano 18 (UDBC) \\
\hline Pouteria lecythidicarpa P.E. Sánchez \& Poveda & Magdalena Valley & Serrano 24 (UDBC) \\
\hline Pouteria lecythidicarpa P.E. Sánchez \& Poveda & Magdalena Valley & Serrano 352 (UDBC) \\
\hline Pouteria lecythidicarpa P.E. Sánchez \& Poveda & Magdalena Valley & Serrano 44 (UDBC) \\
\hline Pouteria lecythidicarpa P.E. Sánchez \& Poveda & Central America & Anderberg et al. 34 (S) \\
\hline Pouteria lucumifolia (Reissek ex Maxim.) T.D. Penn. & Amazon & Richardson et al. 306 (E) \\
\hline Pouteria macrocarpa (Mart.) D. Dietr. & Guyanas & Baraloto, Engel, Chave \& Riera 3095 (CAY) NL110257 \\
\hline Pouteria macrophylla (Lam.) Eyma & Amazon & Seidel et al. $5905(\mathrm{~K})$ \\
\hline Pouteria multiflora (A. DC.) Eyma & Choco & Villa \& Rivaz 257 (BM) \\
\hline Pouteria oblanceolata Pires & Amazon & Garcia 8625 (E) \\
\hline Pouteria oblanceolata Pires & Amazon & Garcia 8634 (E) \\
\hline Pouteria oblanceolata Pires & Amazon & Garcia $8635(\mathrm{E})$ \\
\hline Pouteria oblanceolata Pires & Amazon & Richardson et al. 307 (E) \\
\hline Pouteria platyphylla (A.C. Sm.) Baehni & Catatumbo & Serrano 223 (UDBC) \\
\hline Pouteria platyphylla (A.C. Sm.) Baehni & Choco & Serrano 303 (UDBC) \\
\hline Pouteria platyphylla (A.C. Sm.) Baehni & Magdalena Valley & Serrano 348 (UDBC) \\
\hline Pouteria platyphylla (A.C. Sm.) Baehni & Magdalena Valley & Serrano 350 (UDBC) \\
\hline Pouteria platyphylla (A.C. Sm.) Baehni & Magdalena Valley & Serrano 359 (UDBC) \\
\hline Pouteria platyphylla (A.C. Sm.) Baehni & Amazon & Serrano 55 (UDBC) \\
\hline Pouteria platyphylla (A.C. Sm.) Baehni & Amazon & Richardson et al. 313 (E) \\
\hline Pouteria platyphylla (A.C. Sm.) Baehni & Amazon & Serrano 49 (UDBC) \\
\hline Pouteria procera (Mart.) K. Hammer & Amazon & Richardson et al. 339 (E) \\
\hline Pouteria pubescens (Aubrév. \& Pellegr.) T.D. Penn. & Amazon & Garcia $8636(\mathrm{E})$ \\
\hline Pouteria pubescens (Aubrév. \& Pellegr.) T.D. Penn. & Amazon & $\mathrm{TBC}^{*}$ \\
\hline Pouteria putamen-ovi T.D. Penn. & Amazon & Garcia $8774(\mathrm{E})$ \\
\hline Pouteria putamen-ovi T.D. Penn. & Amazon & $\mathrm{TBC}^{*}$ \\
\hline Pouteria resinosa T.D. Penn & Choco & Serrano 243 (UDBC) \\
\hline Pouteria resinosa T.D. Penn & Choco & Serrano 259 (UDBC) \\
\hline Pouteria reticulata (Engl.) Eyma & Amazon & Serrano 72 (UDBC) \\
\hline Pouteria reticulata (Engl.) Eyma & Central America & Anderberg et al. 7 (S) \\
\hline Pouteria rostrata (Huber) Baehni & Magdalena Valley & Serrano 357 (UDBC) \\
\hline Pouteria sessilis T.D. Penn. & Amazon & Garcia 8637 (E) \\
\hline Pouteria sessilis T.D. Penn. & Amazon & Richardson et al. 321 (E) \\
\hline Pouteria subrotata Cronquist & Amazon & Serrano 112 (UDBC) \\
\hline Pouteria subrotata Cronquist & Amazon & Serrano 145 (UDBC) \\
\hline Pouteria subrotata Cronquist & Amazon & Serrano 151 (UDBC) \\
\hline Pouteria subrotata Cronquist & Central America & Anderberg et al. 48 (S) \\
\hline Pouteria torta (Mart.) Radlk. & Amazon & Serrano 105 (UDBC) \\
\hline Pouteria torta (Mart.) Radlk. & Amazon & Serrano 153 (UDBC) \\
\hline Pouteria torta (Mart.) Radlk. & Amazon & Serrano 50 (UDBC) \\
\hline Pouteria torta (Mart.) Radlk. & Amazon & Serrano 58 (UDBC) \\
\hline Pouteria torta (Mart.) Radlk. & Amazon & Serrano 59 (UDBC) \\
\hline Pouteria torta (Mart.) Radlk. & Amazon & Serrano 69 (UDBC) \\
\hline Pouteria torta (Mart.) Radlk. & Amazon & Serrano 70 (UDBC) \\
\hline Pouteria torta (Mart.) Radlk. & Amazon & Serrano 78 (UDBC) \\
\hline Pouteria torta (Mart.) Radlk. & Amazon & Serrano 90 (UDBC) \\
\hline
\end{tabular}




\begin{tabular}{|c|c|c|}
\hline Taxon & Locality & Collector (herbarium)/GenBank number \\
\hline Pouteria torta (Mart.) Radlk. & Amazon & Anderberg et al. 47 (S) \\
\hline Pouteria torta (Mart.) Radlk. & Guyanas & Baraloto, Engel \& Riera (CAY) NH200224 \\
\hline Pouteria torta (Mart.) Radlk. & Amazon & Richardson et al. $336(\mathrm{E})$ \\
\hline Pouteria torta (Mart.) Radlk. & Amazon & Richardson et al. 338 (E) \\
\hline Pouteria triplarifolia Standl. \& L.O. Williams ex T.D. Penn & Central America & Villa et al. 1304 (BM) \\
\hline Pouteria venosa (Mart.) Baehni & Amazon & Serrano 149 (UDBC) \\
\hline Pouteria venosa (Mart.) Baehni & Amazon & Serrano 76 (UDBC) \\
\hline Pouteria vernicosa T.D. Penn. & Choco & Swenson et al. 738 (S) \\
\hline Pouteria viridis (Pittier) Cronquist & Central America & Faber-Langendoen \& Renteria $1127(\mathrm{~K})$ \\
\hline Pradosia atroviolacea Ducke & Choco & Anderberg et al. 52 (S) \\
\hline Pradosia atroviolacea Ducke & Central America & Lindeman $6743(\mathrm{U})$ \\
\hline Pradosia brevipes (Pierre) T.D. Penn. & Amazon & Cuatrecasas $13988(\mathrm{~K})$ \\
\hline Pradosia cuatrecasasii (Aubrév.) T.D. Penn. & Choco & Baraloto, Engel \& Riera s.n. (CAY) NH200016 \\
\hline Pradosia Liais & Amazon & Serrano 56 (UDBC) \\
\hline Pradosia ptychandra (Eyma) T.D. Penn. & Guyanas & Baraloto, Engel, Chave \& Riera (CAY) NL110310 \\
\hline Pradosia ptychandra (Eyma) T.D. Penn. & Guyanas & Baraloto, Engel, Chave \& Riera 3111 (CAY) NL110514 \\
\hline Pradosia ptychandra (Eyma) T.D. Penn. & Guyanas & Ducke Reserve 05-1829 (K) \\
\hline Pradosia schomburgkiana (A. DC.) Cronquist & Amazon & Isembe 508 (WAG) \\
\hline Pradosia spinosa Ewango \& Breteler & Africa & Harris $1076(\mathrm{U})$ \\
\hline Pradosia surinamensis (Eyma) T.D. Penn. & Guyanas & Harris $1076(\mathrm{U})$ \\
\hline Sapotaceae Juss. & Guyanas & Guy $13^{*}$ \\
\hline Sapotaceae Juss. & Guyanas & Guy $2^{*}$ \\
\hline Sapotaceae Juss. & Guyanas & Guy 24* \\
\hline Sapotaceae Juss. & Guyanas & Guy $26^{*}$ \\
\hline Sapotaceae Juss. & Guyanas & Guy $3^{*}$ \\
\hline Sapotaceae Juss. & Guyanas & Guy $32 *$ \\
\hline Sapotaceae Juss. & Guyanas & Guy $37^{*}$ \\
\hline Sapotaceae Juss. & Guyanas & Guy $47^{*}$ \\
\hline Sapotaceae Juss. & Guyanas & Guy 55* \\
\hline Sapotaceae Juss. & Guyanas & Guy $74 *$ \\
\hline Sapotaceae Juss. & Guyanas & Guy 76* \\
\hline Sapotaceae Juss. & Magdalena Valley & Serrano (UDBC) \\
\hline Sapotaceae Juss. & Magdalena Valley & Serrano (UDBC) \\
\hline Sapotaceae Juss. & Guyanas & $\mathrm{TBC}^{*}$ \\
\hline Sapotaceae Juss. & Choco & Serrano 278 (UDBC) \\
\hline Sapotaceae Juss. & Choco & Serrano 288 (UDBC) \\
\hline Sapotaceae Juss. & Choco & Serrano 315 (UDBC) \\
\hline Sarcaulus brasiliensis (A. DC.) Eyma & Magdalena Valley & Serrano 355 (UDBC) \\
\hline Sarcaulus brasiliensis (A. DC.) Eyma & Amazon & Paniagua et al. 4852 (MO) \\
\hline Sarcaulus brasiliensis (A. DC.) Eyma & Amazon & Richardson et al. 322 (E) \\
\hline Sarcosperma laurinum (Benth.) Hook. $f$. & Asia & Saunders s.n. (S) AM408055 \\
\hline Synsepalum dulcificum (Schumach. \& Thonn.) Daniell & Africa & Welsing et al. 24 (WAG) DQ246697 \\
\hline Synsepalum passargei (Engl.) T.D. Penn. & Africa & Magogo 2452 (UPS) DQ246698 \\
\hline Xantolis siamensis (Fletcher) P. Royen & Asia & Smitairi 1 (L) \\
\hline
\end{tabular}

\section{References}

Albert, J.S., Lovejoy, N.R., Crampton, W.G.R., 2006. Miocene tectonism and the separation of cis- and trans-Andean river basins: evidence from neotropical fishes. J. S. Am. Earth Sci. 21, 14-27.

Anderberg, A.A., Swenson, U., 2003. Evolutionary lineages in Sapotaceae (Ericales): a Cladistic analysis based on ndhF sequence data. Int. J. Plant Sci. 164, 763-773.

Armstrong, K.E., Stone, G.N., Nicholls, J.A., Valderrama Escallón, E., Anderberg, A.A., Smedmark, J., Richardson, J.E., 2014. Patterns of diversification amongst tropical regions compared: a case study in Sapotaceae. Front. Genet. 5, 1-13.

Arrivillaga, J.C., Norris, D.E., Feliciangeli, M.D., Lanzaro, G.C., 2002. Phylogeography of the neotropical sand fly Lutzomyia longipalpis inferred from mitochondrial DNA sequences. Infect. Genet. Evol. 2, 83-95.

Bacon, C.D., Silvestro, D., Jaramillo, C., Smith, B.T., Chakrabarty, P., Antonelli, A., 2015 Biological evidence supports an early and complex emergence of the Isthmus of Panama. Proc. Natl. Acad. Sci. U. S. A. 112, 6110-6115.

Bacon, C.D., Velasquez-Puentes, F.J., Hoorn, C., Antonelli, A., 2018. Iriarteeae palms tracked the uplift of Andean Cordilleras. J. Biogeogr. 7, 1653-1663.

Bartish, I.V., Antonelli, A., Richardson, J.E., Swenson, U., 2011. Vicariance or longdistance dispersal: historical biogeography of the pantropical subfamily Chrysophylloideae (Sapotaceae). J. Biogeogr. 38, 177-190.

Bernal, R., Gradstein, S.R., Celis, M., 2016. Catálogo de Plantas y Líquenes de Colombia. Universidad Nacional de Colombia, Sede Bogotá, Facultad de Ciencias, Instituto de Ciencias Naturales.

Bremer, B., 2009. Asterids. In: Hedges, S.B., Kumar, S. (Eds.), The Timetree of Life. Oxford University Press pp, Oxford, UK, pp. 177-187.

Bremer, K., Friis, E., Bremer, B., 2004. Molecular phylogenetic dating of asterids flowering plants shows early cretaceous diversification. Syst. Biol. 53, 496-505.

Burnham, R.J., Graham, A., 1999. The history of neotropical vegetation: new developments and status. Ann. Mo. Bot. Gard. 86, 546-589.
Burnham, R.J., Johnson, K.R., 2004. South American paleobotany and the origins of neotropical rainforests. Phil Trans R Soc London series B 359, 1595-1610.

Coates, A.G., Obando, J.A., 1996. The geologic evolution of the Central American isthmus, p. 21-56. In: Jackson, J.B.C., Budd, A.F., Coates, A.G. (Eds.), Evolution and Environment in Tropical America. Chicago University, Chicago, EEUU.

Cody, S., Richardson, J.E., Rull, V., Ellis, C., Pennington, R.T., 2010. The great American biotic interchange revisited. Ecography 33, 1-7.

Colinvaux, P.A., Irion, G., Rasanen, M.E., Bush, M.B., 2001. A paradigm to be discarded: geological and paleoecological data falsify the Haffer \& Prance refuge hypothesis of Amazonian speciation. Amazoniana 16, 609-646.

Darriba, D., Taboada, G.L., Doallo, R., Posada, D., 2012. jModelTest 2: more models, new heuristics and parallel computing. Nat. Methods 9, 772.

De-Silva, D.L., Elias, M., Willmott, K., Mallet, J., Day, J.J., 2016. Diversification of clearwing butterflies with the rise of the Andes. J. Biogeogr. 43, 44-58.

Dexter, K.G., Lavin, M., Torke, B.M., Twyford, A.D., Kursar, T.A., Coley, P.D., Pennington, R.T., 2017. Dispersal assembly of rain forest tree communities across the Amazon basin. Proc. Natl. Acad. Sci. U. S. A. 114, 2645-2650.

Díaz de Gamero, M., 1996. The changing course of the Orinoco River during the Neogene: a review. Palaeogeogr. Palaeoclimatol. Palaeoecol. 123, 385-402.

Drummond, A.J., Rambaut, A., 2007. BEAST: Bayesian Evolutionary Analysis by Sampling Trees. BMC Evol. Biol. 7, 214.

Faria, D., Pirani, J.R., Ribeiro, J., Nylinder, S., Terra-Araujo, M., Vieira, P., Swenson, U., 2017. Towards a natural classification of Sapotaceae subfamily Chrysophylloideae in the Neotropics. Bot. J. Linn. Soc. 185, 27-55.

Farris, D.W., Jaramillo, C., Bayona, G., Restrepo-Moreno, S.A., Montes, C., Cardona, A., Valencia, V., 2011. Fracturing of the Panamanian Isthmus during initial collision with South America. Geology 39, 1007-1010.

Fine, P.V.A., Zapata, F., Daly, D.C., 2014. Investigating processes of neotropical rain forest tree diversification by examining the evolution and historical biogeography of the Protieae (Burseraceae). Evolution 68, 1988-2004. 
Gentry, A.H., 1982. Neotropical floristic diversity: Phytogeographical connections between Central and South America: Pleistocene climatic fluctuations, or an accident of the Andean orogeny? Ann. Mo. Bot. Gard. 69, 557-593.

Gonzalez, M.A., Baraloto, C., Engel, J., Mori, S.A., Pétronelli, P., Riéra, B., Roger, A., Thébaud, C., Chave, J., 2009. Identification of Amazonian trees with DNA barcodes. PLoS One 4, e7483.

Graham, A., 2011. The age and diversification of terrestrial new world ecosystems through cretaceous and Cenozoic time. Amer J. Bot. 98, 336-351.

Gregory-Wodzicki, K.M., 2000. Uplift history of the central and northern Andes: a review. Geol. Soc. Am. Bull. 112, 1091-1105.

Haffer, J., 1969. Speciation in Amazonian forest birds. Science 165, 131-137.

Hall, T.A., 1999. BioEdit: a user-friendly biological sequence alignment editor and analysis program for Windows 95/98/NT. Nucleic Acids Symp. Ser. 41, 95-98.

Hazzi, N.A., Moreno, J.S., Ortiz-Movliav, C., Palacio, R.D., 2018. Biogeographic regions and events of isolation and diversification of the endemic biota of the tropical Andes Proc. Natl. Acad. Sci. U. S. A. 115, 7985-7990.

Hernández-Camacho, J., Hurtado, A., Ortiz, R., Walschburger, T., 1992. Unidades biogeográficas de Colombia. In: Halfter, G. (Ed.), La diversidad biológica de Iberoamérica. Instituto de Ecología, A. C., México, pp. 105-173.

Ho, S.Y.W., 2007. Calibrating molecular estimates of substitution rates and divergence times in birds. J. Avian Biol. 38, 409-414.

Ho, S.Y.W., Phillips, M.J., 2009. Accounting for calibration uncertainty in phylogenetic estimation of evolutionary divergence times. Syst. Biol. 58, 367-380.

Höhna, S., Landis, M., Heath, T., Boussau, B., Lartillot, N., Moore, B., Huelsenbeck, J., Ronquist, F., 2016. RevBayes: Bayesian phylogenetic inference using graphical models and an interactive model-specification language. Syst. Biol. 65, 726-736.

Hoorn, C., Flantua, S., 2015. An early start for the Panama land bridge. Science 348 , 186-187.

Hoorn, C., Guerrero, J., Sarmiento, G.A., Lorente, M.A., 1995. Andean tectonics as a cause for changing drainage patterns in Miocene northern South America. Geology 23, 237-240.

Hoorn, C., Wesselingh, F.P., ter Steege, H., Bermudez, M.A., Mora, A., Sevink, J., Antonelli, A., 2010. Amazonia through time: Andean uplift, climate change, landscape evolution and biodiversity. Science 330, 927-931.

Hoorn, C., van der Ham, R., Parra, F., Salamanca, S., ter Steege, H., Banks, H., Star, W., van Heuven, B., Langelaan, R., Carvalho, F., Rodriguez-Forero, G., Lagomarsino, L. 2019. Going north and south: the biogeographic history of two Malvaceae in the wake of Neogene Andean uplift and connectivity between the Americas. Rev. Palaeobot. Palynol. 264, 90-109.

Huelsenbeck, J.P., Ronquist, F., 2001. MRBAYES: Bayesian inference of phylogeny. Bioinformatics 17, 754-755.

IDEAM, IGAC, IAvH, Invemar, I., Sinchi e IIAP, 2007. Ecosistemas continentales, costeros y marinos de Colombia. Instituto de Hidrología, Meteorología y Estudios Ambientales, Instituto Geográfico Agustín Codazzi, Instituto de Investigación de Recursos Biológicos Alexander von Humboldt, Instituto de Investigaciones Ambientales del Pacífico Jhon von Neumann. Instituto de Investigaciones Marinas y Costeras José Benito Vives De Andréis e Instituto Amazónico de Investigaciones Científicas Sinchi. Bogotá, D. C, 276 p. + 37 hojas cartográficas.

Jan du Chêne, R.E., Onyike, M.S., Sowunmi, M.A., 1978. Some new Eocene pollen of the Ogwashi-Asaba Formation, South-Eastern Nigeria. Rev. Esp. Micropaleontol. 10, 285-322.

Kearse, M., Moir, R., Wilson, A., Stones-Havas, S., Cheung, M., Sturrock, S. Drummond, A., 2012. Geneious basic: an integrated and extendable desktop software platform for the organization and analysis of sequence data. Bioinformatics 28, 1647-1649.

Koenen, E.J.M., Clarkson, J.J., Pennington, T.D., Chatrou, L.W., 2015. Recently evolved diversity and convergent radiations of rainforest mahoganies (Meliaceae) shed new light on the origins of rainforest hyperdiversity. New Phytol. 207, 327-339.

Kroonenberg, S., Bakker, J., Van der Wiel, M., 1990. Late Cenozoic uplift and paleogeography of the Colombian Andes: constraints on the development of highandean biota. Geologie 69, 279-290.

Lasso, C.A., Morales-Betancourt, M.A., Escobar-Martínez, I.D. (Eds.), 2018. Biodiversidad de la sierra de La Macarena, Meta, Colombia. Parte I. Ríos Guayabero medio, bajo Losada y bajo Duda. Serie Editorial Fauna Silvestre Neotropical. Instituto de Investigación de Recursos Biológicos Alexander von Humboldt. Bogotá, D. C., Colombia, p. 329.

Madriñán, S., Cortés, A.J., Richardson, J.E., 2013. Páramo is the world's fastest evolving and coolest biodiversity hotspot. Front. Genet. 4, 192. 10.

Matzke Nicholas, J., 2018. BioGeoBEARS: BioGeography with Bayesian (and likelihood) Evolutionary Analysis with R Scripts. version 1.1.1, published on GitHub on November 6.

Meyer, A., Román-Palacios, C., 2018. BAMM gives misleading rate estimates in simulated and empirical datasets. Evolution 72, 2257-2266.

Miller, M.A., Pfeiffer, W., Schwartz, T., 2010. Creating the CIPRES Science Gateway for inference of large phylogenetic trees. In: Proceedings of the Gateway Computing Environments Workshop (GCE) (pp. 1-8). New Orleans, LA.

Moonlight, P.W., Richardson, J.E., Tebbitt, M.C., Thomas, D.C., Hollands, R., Peng, C.I., Hughes, M., 2015. Continental-scale diversification patterns in a megadiverse genus: the biogeography of Neotropical Begonia. J. Biogeogr. 42, 1137-1149.

Moore, B.R., Hohna, S., May, M.R., Rannala, B., Huelsenbeck, J.P., 2016. Critically evaluating the theory and performance of Bayesian analysis of macroevolutionary mixtures. Proc. Natl. Acad. Sci. U. S. A. 113, 9569-9574.

Mora, A., Baby, P., Roddaz, M., Parra, M., Brusset, S., Hermoza, W., Espurt, N., 2010. Tectonic history of the Andes and sub-Andean zones: Implications for the development of the Amazon drainage basin. In: Hoorn, C., Wesselingh, F.P. (Eds.),
Amazonia-Landscape and Species Evolution: A Look into the Past. Wiley-Blackwell, Oxford, pp. 38-60.

Moritz, C., Patton, J.L., Schneider, C.J., Smith, T.B., 2000. Diversification of rainforest Faunas: an Integrated Molecular Approach. Annu. Rev. Ecol. Syst. 31, 533-563.

Morrone, J.J., 2001. Biogeografía de América Latina y el Caribe. Manuales y Tesis SEA. 148.

Olson, D.M., Dinerstein, E., Wikramanayake, E.D., Burgess, N.D., Powell, G.V.N., Underwood, E.C., Kassem, K.R., 2001. Terrestrial ecoregions of the world: a new map of life on Earth. Bioscience 51, 933-938.

Pennington, T.D., 1991. The Genera of Sapotaceae. Royal Botanic Gardens Kew, New York Botanical Garden.

Pennington, R.T., Dick, C.W., 2004. The role of immigrants in the assembly of the south American rainforest tree flora. Phil Trans R Soc London series B. Biol. Sci. 359, 1611-1622.

Pennington, R.T., Lavin, M., 2016. The contrasting nature of woody plant species in different neotropical forest biomes reflects differences in ecological stability. New Phytol. 210, 25-37.

Pennington, R.T., Lavin, M., Sarkinen, T., Lewis, G.P., Klitgaard, B.B., Hughes, C.E., 2010. Contrasting plant diversification histories within the Andean biodiversity hotspot. Proc. Natl. Acad. Sci. U. S. A. 107, 13783-13787.

Pérez-Escobar, O.A., Chomicki, G., Condamine, F.L., Karremans, A.P., Bogarín, D., Matzke, N.J., Silvestro, D., Antonelli, A., 2017. Recent origin and rapid speciation of Neotropical orchids in the world's richest plant biodiversity hotspot. New Phytol. 215, 891-905.

Pérez-Escobar, O., Lucas, E., Jaramillo, C., Monro, A., Morris, S., Bogarín, D., Antonelli, A., 2019. The Origin and diversification of the Hyperdiverse Flora in the Chocó Biogeographic Region. Front. Plant Sci. 9.

Pirie, M.D., Chatrou, L.W., Mols, J.B., Erkens, R.H.J., Oosterhof, J., 2006. 'Andeancentred' genera in the short-branch clade of Annonaceae: Testing biogeographic hypotheses using phylogeny reconstruction and molecular dating. J. Biogeogr. 33, $31-46$.

Prance, G.T., 1973. Phytogeographic support for the theory of Pleistocene forest refuges in the Amazon Basin, based upon evidence from distribution pattern in Caryocaraceae, Chry- Solanaceae, Dichapetalaceae and Lecythidaceae. Acta Amazon. 3, 5-28.

\section{$\mathrm{R}$ Core Team. R, 2018. A language and environment for statistical computing. $\mathrm{R}$} Foundation for Statistical Computing, Vienna, Austria. https://www.R-project.org/.

Rabosky, D.L., 2014. Automatic detection of key innovations, rateshifts, and diversitydependence on phylogenetic trees. PLoS One 9, e89543.

Rabosky, D.L., 2019. Phylogenies and diversification rates: Variance cannot be Ignored. Syst. Biol. 68, 538-550.

Rabosky, D., Grundler, M., Anderson, C., Title, P., Shi, J., Brown, J., Huang, H., Larson, J., 2014. BAMMtools: an R package for the analysis of evolutionary dynamics on phylogenetic trees. Methods Ecol. Evol. 5, 701-707.

Rabosky, D., Jonathan, M., Jonathan, C., 2017. Is BAMM Flawed? Theoretical and Practical concerns in the Analysis of Multi-Rate diversification Models. Syst. Biol. 66, 477-498.

Rambaut, A., Suchard, M.A., Xie, D., Drummond, A.J., 2014. Tracer v1.6.

Ree, R.H., Sanmartín, I., 2018. Conceptual and statistical problems with the DEC $+J$ model of founder-event speciation and its comparison with DEC via model selection. J. Biogeogr. 45 (4), 741-749.

Reynel, C., Pennington, T., Särkinen, T., 2013. Cómo se formó la diversidad ecológica del Perú. Lima, Perú.

Richardson, J.E., Pennington, T., Pennington, T.D., Hollingsworth, P., 2001. Rapid diversification of a species-rich genus of neotropical rain forest trees. Science 293, 2242-2245.

Richardson, J.E., Bakar, A.M., Tosh, J., Armstrong, K., Smedmark, J., Anderberg, A.A., Wilkie, P., 2014. The influence of tectonics, sea-level changes and dispersal on migration and diversification of Isonandreae (Sapotaceae). Bot. J. Linn. Soc. 174, $130-140$.

Richardson, J.E., Whitlock, B.A., Meerow, A.W., Madriñán, S., 2015. The age of chocolate: a diversification history of Theobroma and Malvaceae. Front. Genet. 10.

Richardson, J.E., Madriñán, S., Gómez, M.C., Valderrama, E., Luna, J., Banda, R.K., Serrano, J., Torres, M.F., Jara, O.A., Aldana, A.M., Cortés, B.R., Sánchez, D., Montes, C., 2018. Using dated molecular phylogenies to reconstruct geological, climatic and biological history: examples from Colombia. Geol. J. 10.

Ronquist, F., Huelsenbeck, J.P., 2003. MRBAYES 3: Bayesian phylogenetic inference under mixed models. Bioinformatics 19, 1572-1574.

Rull, V., 1997. Sequence analysis of Western Venezuelan cretaceous to Eocene sediments using palynology. Chrono-palaeoenvironmental and palaeovegetational approaches. Palynology 21, 79-90.

Rull, V., 2000. Ecostratigraphic study of Paleocene and early Eocene palynological cyclicity in northern South America. Palaios 15, 14-24.

Sánchez-C, D., Richardson, J.E., Hart, M., Serrano, J., Cardenas, D., Cortés-B, R., 2021. A plea to barcode type specimens: an example from Micropholis (Sapotaceae) (Submitted to Taxon).

Serrano, J., Richardson, J.E., Pennington, T.D., Cortés-BR, Cárdenas D., Elliot, A., Jiménez, I., 2018. Biotic homogeneity of putative biogeographic units in the Neotropics: a test with Sapotaceae. Divers. Distrib. 24, 1-15.

Simpson, G.G., 1980. Splendid Isolation: The Curious History of South American Mammals. Yale University Press, Yale, MI.

Swenson, U., Richardson, J.E., Bartish, I.V., 2008. Multi-gene phylogeny of the pantropical subfamily Chrysophylloideae (Sapotaceae): evidence of generic polyphyly and extensive morphological homoplasy. Cladistics 24, 1006-1031.

Trénel, P., Gustafsson, M.H.G., Baker, W.J., Asmussen-Lange, C.B., Dransfield, J., Borchsenius, F., 2007. Mid-Tertiary dispersal, not Gondwanan vicariance explains 
distribution patterns in the wax palm subfamily (Ceroxyloideae: Arecaceae). Mol. Phylogenet. Evol. 45, 272-288.

Whinnett, A., Zimmermann, M., Willmott, K.R., Herrera, N., Mallarino, R., Simpson, Mallet, J., 2005. Strikingly variable divergence times inferred across an Amazonian butterfly 'suture zone'. Proceedings of the Royal Society, London, B 272, 2525-2533.

Winship, T., 1990. Paleobiogeographic relationships of angiosperms from the cretaceous and early Tertiary of the north American area. Bot. Rev. 56, 279-417.

Winterton, C., Richardson, J.E., Hollingsworth, M., Clark, A., Zamora, N., Pennington, R.

T., 2014. Historical biogeography of the neotropical legume genus Dussia: The
Andes, the Panama Isthmus and the Chocó. In: Stevens, W.D., Montiel, O.M., Raven, P. (Eds.), Paleobotany and Biogeography: A Festschrift for Alan Graham in his 80th Year. Missouri Botanical Garden Press pp, St Louis, MO, pp. 389-404.

Wolfe, J.A., 1975. Some aspects of plant geography of the northern hemisphere during the late cretaceous and Tertiary. Ann. Mo. Bot. Gard. 62, 264-279.

Wortel, R., 1984. Spatial and temporal variations in the Andean subduction zone. J. Geol. Soc. 141, 783-791. 


\section{Update 1 of 2 \\ Global and Planetary Change \\ Volume, Issue , , Page}

DOI: https://doi.org/10.1016/j.gloplacha.2021.103576 


\title{
Corrigendum to "Andean orogeny and the diversification of lowland neotropical rain forest trees: A case study in Sapotaceae" [Global and Planetary Change 201 (2021) 103481]
}

\author{
Julieth Serrano $^{\text {a, }}$, James E. Richardson ${ }^{\text {b, }}$, Richard I. Milne ${ }^{\text {d }}$, G. Ariadna Mondragon ${ }^{\text {e }}$, \\ Julie A. Hawkins ${ }^{f}$, Igor V. Bartish ${ }^{g}$, Mailyn Gonzalez ${ }^{\text {h }}$, Jerome Chave ${ }^{i}$, Santiago Madrinán ${ }^{e, 1}$, \\ Dairon Cárdenas ${ }^{\mathrm{j}}$, S. Dayana Sanchez ${ }^{\mathrm{k}}$, Rocio Cortes-B ${ }^{\mathrm{k}}$, R. Toby Pennington ${ }^{\mathrm{a}, \mathrm{c}}$ \\ ${ }^{a}$ Geography Department, College of Life and Environmental Sciences, University of Exeter, Rennes Drive, Exeter EX4 4RJ, UK \\ ${ }^{\mathrm{b}}$ Facultad de Ciencias Naturales, Universidad del Rosario, Bogotá, D.C., Colombia \\ ${ }^{\mathrm{c}}$ Tropical Diversity Section, Royal Botanic Garden Edinburgh, 20a Inverleith Row, Edinburgh EH3 5LR, UK \\ ${ }^{\mathrm{d}}$ Institute of Molecular Plant Sciences, Kings Building, West Mains Road, University of Edinburgh, EH9 3JW, UK \\ e Laboratorio de Botánica y Sistemática, Universidad de los Andes, Apartado Áereo 4976, Bogotá, D.C., Colombia \\ ${ }^{\mathrm{f}}$ School of Biological Sciences, University of Reading, Reading RG6 6BX, UK \\ ${ }^{g}$ Department of Population Ecology, Institute of Botany, Academy of Sciences, CZ-25243 Prìhonice, Czech Republic \\ ${ }^{\mathrm{h}}$ Instituto Alexander von Humboldt, Avenida Paseo Bolivar (Circunvalar) 16-20, Bogotá, D.C., Colombia \\ ${ }^{\mathrm{i}}$ Laboratoire Evolution et Diversite Biologique UMR 5174, Universite Paul Sabatier/CNRS, 31062 Toulouse, France \\ j Instituto Amaźonico de Investigaciones Científicas Sinchi, Calle 20 \# 5-44, Bogotá, D.C., Colombia \\ ${ }^{\mathrm{k}}$ Herbario Forestal, Universidad Distrital, Carrera 5 Este \# 15-82, Bogotá D.C., Colombia \\ ${ }^{1}$ Jardín Botánico de Cartagena "Guillermo Piñeres", Turbaco, Bolívar, Colombia
}

A R T I C L E I N F O

Editor: Howard Falcon-Lang

The authors apologise for omitting a full record of GenBank numbers, and a complete list is provided here together with an update on the Acknowledgements section.

Appendix 1. List of specimens included in the phylogenetic analyses. Internal transcribed spacers of rDNA (ITS) sequence data was generated. A total of 146 Chrysophylloideae accessions were collected in the Chocó, Magdalena valley, Catatumbo, Amazon and Macarena lowland rain forests of Colombia. The remaining sequences were generated by Roosevelt Garcia, and obtained from previous phylogenetic studies on Chrysophylloideae (Sánchez-C. et al., 2021; Gonzalez et al., 2009; Swenson et al., 2008), and GenBank.

\begin{tabular}{llll}
\hline Taxon & Locality & Colector & Genebank \\
\hline $\begin{array}{c}\text { Breviea sericea (A. Chev.) } \\
\text { Aubrév. \& Pellegr. }\end{array}$ & Africa & Letouzey 8319 (P) & DQ246666 \\
& & Anderberg et al. 20 (S) & EF558614
\end{tabular}

(continued on next column) (continued)

\begin{tabular}{|c|c|c|c|}
\hline Taxon & Locality & Colector & Genebank \\
\hline $\begin{array}{l}\text { Chromolucuma rubriflora } \\
\text { Ducke }\end{array}$ & $\begin{array}{l}\text { Central } \\
\text { America }\end{array}$ & & \\
\hline $\begin{array}{l}\text { Chrysophyllum } \\
\text { argenteum Jacq. }\end{array}$ & Chocó & Dick 81 (STRI) & AY635511 \\
\hline $\begin{array}{l}\text { Chrysophyllum } \\
\text { argenteum Jacq. }\end{array}$ & $\begin{array}{l}\text { Central } \\
\text { America }\end{array}$ & Dick 80 (STRI) & AY635513 \\
\hline $\begin{array}{l}\text { Chrysophyllum } \\
\text { argenteum Jacq. }\end{array}$ & Chocó & Dick 85 (STRI) & AY635515 \\
\hline $\begin{array}{l}\text { Chrysophyllum } \\
\text { argenteum Jacq. }\end{array}$ & Guyanas & $\begin{array}{l}\text { Baraloto, Engel \& Riera } \\
\text { (CAY) }\end{array}$ & FJ037865 \\
\hline $\begin{array}{l}\text { Chrysophyllum } \\
\text { argenteum Jacq. }\end{array}$ & $\begin{array}{l}\text { Central } \\
\text { America }\end{array}$ & Dick 79 (STRI) & JX987536.1 \\
\hline $\begin{array}{l}\text { Chrysophyllum } \\
\text { argenteum Jacq. }\end{array}$ & Chocó & Dick 87 (STRI) & JX987537 \\
\hline $\begin{array}{l}\text { Chrysophyllum } \\
\text { argenteum Jacq. }\end{array}$ & Chocó & Dick 82 (STRI) & JX987538 \\
\hline
\end{tabular}

(continued on next page)

DOI of original article: https://doi.org/10.1016/j.gloplacha.2021.103481.

* Corresponding author.

E-mail address: juliserranoa@yahoo.com (J. Serrano). 
(continued)

\begin{tabular}{|c|c|c|c|}
\hline Taxon & Locality & Colector & Genebank \\
\hline $\begin{array}{l}\text { Chrysophyllum } \\
\text { argenteum Jacq. }\end{array}$ & Chocó & Serrano 286 (UDBC) & MZ329867 \\
\hline $\begin{array}{l}\text { Chrysophyllum } \\
\text { argenteum Jacq. }\end{array}$ & Macarena & Serrano $n n 367^{\mathrm{a}}$ & MZ329868 \\
\hline $\begin{array}{l}\text { Chrysophyllum } \\
\text { argenteum Jacq. }\end{array}$ & Chocó & Serrano 280 (UDBC) & MZ329869 \\
\hline $\begin{array}{l}\text { Chrysophyllum } \\
\text { argenteum Jacq. }\end{array}$ & Guyanas & Baraloto 3203 (CAY) & FJ037864 \\
\hline $\begin{array}{l}\text { Chrysophyllum } \\
\text { bangweolense R.E. Fr. }\end{array}$ & Africa & Malaisse 9600 (WAG) & AY552152.1 \\
\hline $\begin{array}{l}\text { Chrysophyllum bicolor } \\
\text { Poir. }\end{array}$ & West Indies & JP57 (DAV) & JF912987 \\
\hline $\begin{array}{l}\text { Chrysophyllum } \\
\text { boivinianum (Pierre) } \\
\text { Baehni }\end{array}$ & Africa & $\begin{array}{l}\text { McPherson 14,426 } \\
\text { (MO, P, WAG) }\end{array}$ & DQ246667.1 \\
\hline $\begin{array}{l}\text { Chrysophyllum } \\
\text { bombycinum T.D. } \\
\text { Penn. }\end{array}$ & Amazon & Serrano 118 (UDBC) & MZ329972 \\
\hline $\begin{array}{l}\text { Chrysophyllum } \\
\text { bombycinum T.D. } \\
\text { Penn. }\end{array}$ & Amazon & Serrano 121 (UDBC) & MZ329973 \\
\hline $\begin{array}{l}\text { Chrysophyllum } \\
\text { bombycinum T.D. } \\
\text { Penn. }\end{array}$ & Amazon & Serrano 120 (UDBC) & MZ329974 \\
\hline $\begin{array}{l}\text { Chrysophyllum } \\
\text { bombycinum T.D. } \\
\text { Penn. }\end{array}$ & Amazon & Serrano 135 (UDBC) & MZ329975 \\
\hline $\begin{array}{l}\text { Chrysophyllum } \\
\text { bombycinum T.D. } \\
\text { Penn. }\end{array}$ & Amazon & Serrano 139 (UDBC) & MZ329976 \\
\hline $\begin{array}{l}\text { Chrysophyllum } \\
\text { bombycinum T.D. } \\
\text { Penn. }\end{array}$ & Amazon & Garcia 8671 (E) & MZ470016 \\
\hline $\begin{array}{l}\text { Chrysophyllum } \\
\text { bombycinum T.D. } \\
\text { Penn. }\end{array}$ & Amazon & Garcia $8673(\mathrm{E})$ & MZ470017 \\
\hline Chrysophyllum cainito $L$. & West Indies & $\begin{array}{l}\text { Chantaranothai } 2304 \\
\text { (KKU) }\end{array}$ & AY552153.1 \\
\hline Chrysophyllum cainito L. & $\begin{array}{l}\text { Cauca } \\
\text { Valley }\end{array}$ & $\begin{array}{l}\text { Richardson et al. } 357 \\
\text { (E) }\end{array}$ & MZ469952 \\
\hline $\begin{array}{l}\text { Chrysophyllum } \\
\text { colombianum } \\
\text { (Aubrév.) T.D. Penn. }\end{array}$ & Chocó & Serrano 320 (UDBC) & MZ329873 \\
\hline $\begin{array}{l}\text { Chrysophyllum } \\
\text { colombianum } \\
\text { (Aubrév.) T.D. Penn. }\end{array}$ & Chocó & Serrano 319 (UDBC) & MZ329874 \\
\hline $\begin{array}{l}\text { Chrysophyllum } \\
\text { colombianum } \\
\text { (Aubrév.) T.D. Penn. }\end{array}$ & Amazon & Serrano 98 (UDBC) & MZ329970 \\
\hline $\begin{array}{l}\text { Chrysophyllum } \\
\text { colombianum } \\
\text { (Aubrév.) T.D. Penn. }\end{array}$ & Amazon & Serrano 100 (UDBC) & MZ329971 \\
\hline $\begin{array}{l}\text { Chrysophyllum } \\
\text { colombianum } \\
\text { (Aubrév.) T.D. Penn. }\end{array}$ & Chocó & $\begin{array}{l}\text { Gentry, } \\
\text { Faber-Langendoen \& } \\
\text { Monsalve 56,806 (K) }\end{array}$ & MZ469957 \\
\hline $\begin{array}{l}\text { Chrysophyllum } \\
\text { cuneifolium (Rudge) A. } \\
\text { DC. }\end{array}$ & Guyanas & Mori et al. 19,135 (K) & DQ246668.1 \\
\hline $\begin{array}{l}\text { Chrysophyllum eximium } \\
\text { Ducke }\end{array}$ & Guyanas & Baraloto 3135 (CAY) & FJ037866.1 \\
\hline $\begin{array}{l}\text { Chrysophyllum } \\
\text { fenerivense (Aubrév.) } \\
\text { G.E. Schatz \& L. Gaut }\end{array}$ & Africa & $\begin{array}{l}\text { Rabehovitra et al. } 4223 \\
\text { (MO) }\end{array}$ & DQ246669 \\
\hline $\begin{array}{l}\text { Chrysophyllum imperiale } \\
\text { (Linden ex K. Koch \& } \\
\text { Fintelm.) Benth. \& } \\
\text { Hook. f. }\end{array}$ & $\begin{array}{l}\text { Atlantic } \\
\text { Forest }\end{array}$ & Pennington s.n. (S) & EF558615.1 \\
\hline Chrysophyllum L. & Amazon & Serrano 80 (UDBC) & MZ329882 \\
\hline Chrysophyllum L. & Amazon & Serrano 67 (UDBC) & MZ329958 \\
\hline Chrysophyllum L. & Amazon & Serrano 64 (UDBC) & MZ329959 \\
\hline Chrysophyllum L. & Amazon & Garcia 8647 (E) & MZ469953 \\
\hline Chrysophyllum L. & $\begin{array}{l}\text { Central } \\
\text { America }\end{array}$ & Anderberg et al. $31^{\mathrm{a}}$ & MZ470012 \\
\hline Chrysophyllum L. & Amazon & Garcia $8581(\mathrm{E})$ & MZ470013 \\
\hline Chrysophyllum & Amazon & Serrano 49 (UDBC) & MZ329902 \\
\hline
\end{tabular}

(continued on next column) (continued)

\begin{tabular}{|c|c|c|c|}
\hline Taxon & Locality & Colector & Genebank \\
\hline $\begin{array}{l}\text { Chrysophyllum } \\
\text { lucentifolium Cronquist }\end{array}$ & Amazon & Serrano 62 (UDBC) & MZ329904 \\
\hline \multicolumn{4}{|l|}{ Chrysophyllum } \\
\hline $\begin{array}{l}\text { manaosense (Aubrév.) } \\
\text { T.D. Penn. }\end{array}$ & Chocó & Serrano 296 (UDBC) & MZ329875 \\
\hline \multicolumn{4}{|l|}{ Chrysophyllum } \\
\hline $\begin{array}{l}\text { manaosense (Aubrév.) } \\
\text { T.D. Penn. }\end{array}$ & Amazon & S. coll. $2^{\mathrm{a}}$ & MZ468590 \\
\hline $\begin{array}{l}\text { Chrysophyllum } \\
\text { manaosense (Aubrév.) } \\
\text { T.D. Penn. }\end{array}$ & Amazon & $\begin{array}{l}\text { Richardson et al. } 334 \\
\text { (E) }\end{array}$ & MZ469955 \\
\hline \multicolumn{4}{|l|}{ Chrysophyllum } \\
\hline $\begin{array}{l}\text { mexicanum Brandegee } \\
\text { ex Standl. }\end{array}$ & Mexico & JP341 (DAV) & JF912989 \\
\hline \multicolumn{4}{|l|}{ Chrysophyllum } \\
\hline $\begin{array}{l}\text { mexicanum Brandegee } \\
\text { ex Standl. }\end{array}$ & Mexico & JP398 (DAV) & JF912990 \\
\hline \multicolumn{4}{|l|}{ Chrysophyllum } \\
\hline $\begin{array}{l}\text { mexicanum Brandegee } \\
\text { ex Standl. }\end{array}$ & Mexico & JP256 (DAV) & JF912991 \\
\hline $\begin{array}{l}\text { Chrysophyllum oliviforme } \\
\text { L. }\end{array}$ & West Indies & $\begin{array}{l}\text { Guttiérrez \& Nilsson } 1 \\
\text { (S) }\end{array}$ & DQ246670 \\
\hline $\begin{array}{l}\text { Chrysophyllum } \\
\text { pauciflorum Lam }\end{array}$ & West Indies & JP56 (DAV) & JF912986 \\
\hline $\begin{array}{l}\text { Chrysophyllum } \\
\text { pomiferum (Eyma) T. } \\
\text { D. Penn. }\end{array}$ & $\begin{array}{l}\text { Magdalena } \\
\text { Valley }\end{array}$ & $\begin{array}{l}\text { Pennington et al. } \\
12,386^{\mathrm{a}}\end{array}$ & MZ469956 \\
\hline $\begin{array}{l}\text { Chrysophyllum prieurii A. } \\
\text { DC. }\end{array}$ & Amazon & $\begin{array}{l}\text { Richardson et al. } 326 \\
\text { (E) }\end{array}$ & MZ469958 \\
\hline $\begin{array}{l}\text { Chrysophyllum prieurii A. } \\
\text { DC. }\end{array}$ & Amazon & Garcia $8583(\mathrm{E})$ & MZ469982 \\
\hline $\begin{array}{l}\text { Chrysophyllum prieurii A. } \\
\text { DC. }\end{array}$ & Guyanas & $\begin{array}{l}\text { Baraloto, Engel \& } \\
\text { Chave s.n. (CAY) }\end{array}$ & P01860227 \\
\hline \multicolumn{4}{|l|}{ Chrysophyllum } \\
\hline $\begin{array}{l}\text { pruniforme Pierre ex } \\
\text { Engl. }\end{array}$ & Africa & Jongkind 3762 (WAG) & DQ246671 \\
\hline $\begin{array}{l}\text { Chrysophyllum } \\
\text { roxburghii G. Don }\end{array}$ & Africa & $\begin{array}{l}\text { Solo \& Randrianasolo } \\
33 \text { (WAG) }\end{array}$ & DQ246672 \\
\hline $\begin{array}{l}\text { Chrysophyllum } \\
\text { sanguinolentum } \\
\text { (Pierre) Baehni }\end{array}$ & Guyanas & Baraloto 3016 (CAY) & FJ037868 \\
\hline $\begin{array}{l}\text { Chrysophyllum } \\
\text { sanguinolentum } \\
\text { (Pierre) Baehni }\end{array}$ & Guyanas & Baraloto 3196 (CAY) & FJ037869.2 \\
\hline $\begin{array}{l}\text { Chrysophyllum } \\
\text { sanguinolentum } \\
\text { (Pierre) Baehni }\end{array}$ & Amazon & Garcia 8645 (E) & MZ469968 \\
\hline $\begin{array}{l}\text { Chrysophyllum } \\
\text { sanguinolentum } \\
\text { (Pierre) Baehni }\end{array}$ & Amazon & $\begin{array}{l}\text { Richardson et al. } 305 \\
\text { (E) }\end{array}$ & MZ469980 \\
\hline $\begin{array}{l}\text { Chrysophyllum } \\
\text { sanguinolentum } \\
\text { (Pierre) Baehni }\end{array}$ & Amazon & $\begin{array}{l}\text { Richardson et al. } 310 \\
\text { (E) }\end{array}$ & MZ470011 \\
\hline $\begin{array}{l}\text { Chrysophyllum } \\
\text { sanguinolentum } \\
\text { (Pierre) Baehni }\end{array}$ & Amazon & $\begin{array}{l}\text { Richardson et al. } 348 \\
\text { (E) }\end{array}$ & MZ470015 \\
\hline $\begin{array}{l}\text { Chrysophyllum } \\
\text { sanguinolentum } \\
\text { (Pierre) Baehni }\end{array}$ & Amazon & Garcia 8236 (E) & MZ470018 \\
\hline $\begin{array}{l}\text { Chrysophyllum scalare } T \text {. } \\
\text { D. Penn. }\end{array}$ & Amazon & $\begin{array}{l}\text { Richardson et al. } 320 \\
\text { (E) }\end{array}$ & MZ470014 \\
\hline $\begin{array}{l}\text { Chrysophyllum } \\
\text { sparsiflorum Klotzsch } \\
\text { ex Miq. }\end{array}$ & Amazon & GUY71 $^{\mathrm{a}}$ & DQ021882 \\
\hline $\begin{array}{l}\text { Chrysophyllum } \\
\text { venezuelanense } \\
\text { (Pierre) T.D. Penn. }\end{array}$ & Chocó & Ståhl et al. 5755 (S) & DQ246673 \\
\hline $\begin{array}{l}\text { Diploon cuspidatum } \\
\text { (Hoehne) Cronquist }\end{array}$ & Guyanas & $\begin{array}{l}\text { Pennington et al. } \\
13,843(\mathrm{U})\end{array}$ & DQ246676 \\
\hline $\begin{array}{l}\text { Diploon cuspidatum } \\
\text { (Hoehne) Cronquist }\end{array}$ & Guyanas & Baraloto 3209 (CAY) & FJ037870 \\
\hline $\begin{array}{l}\text { Eberhardtia aurata } \\
\quad \text { (Pierre ex Dubard) } \\
\text { Lecomte }\end{array}$ & Asia & Hao 534 (S) & EF558617 \\
\hline $\begin{array}{l}\text { Ecclinusa guianensis } \\
\text { Eyma }\end{array}$ & Amazon & Ducke 05-906 (K) & DQ246677 \\
\hline
\end{tabular}


(continued)

\begin{tabular}{|c|c|c|c|}
\hline Taxon & Locality & Colector & Genebank \\
\hline $\begin{array}{l}\text { Ecclinusa lanceolata } \\
\text { (Mart. \& Eichler) } \\
\text { Pierre }\end{array}$ & Amazon & $\begin{array}{l}\text { Richardson et al. } 314 \\
\text { (E) }\end{array}$ & MZ470007 \\
\hline $\begin{array}{l}\text { Ecclinusa lanceolata } \\
\text { (Mart. \& Eichler) } \\
\text { Pierre }\end{array}$ & Amazon & Garcia 8689 (E) & MZ470008 \\
\hline $\begin{array}{l}\text { Ecclinusa lanceolata } \\
\text { (Mart. \& Eichler) } \\
\text { Pierre }\end{array}$ & Amazon & Garcia 8675 (E) & MZ470009 \\
\hline Ecclinusa Mart. & Chocó & Serrano 309 (UDBC) & MZ329966 \\
\hline Ecclinusa ramiflora Mart. & Guyanas & Irwing et al. 55,081 (S) & DQ246678 \\
\hline Ecclinusa ramiflora Mart. & Macarena & Serrano 170 (UDBC) & MZ329964 \\
\hline Ecclinusa ramiflora Mart. & Macarena & Serrano 177 (UDBC) & MZ329965 \\
\hline Ecclinusa ramiflora Mart. & Macarena & Serrano $n n 360^{\mathrm{a}}$ & MZ468579 \\
\hline Ecclinusa ramiflora Mart. & Macarena & Serrano nn $361^{\mathrm{a}}$ & MZ468580 \\
\hline Ecclinusa ramiflora Mart. & Chocó & Monsalve B1319 (K) & MZ470010 \\
\hline Elaeoluma Baill. & Amazon & Serrano 131 (UDBC) & MZ329963 \\
\hline $\begin{array}{l}\text { Elaeoluma glabrescens } \\
\text { (Mart. \& Eichler) } \\
\text { Aubrév. }\end{array}$ & $\begin{array}{l}\text { Central } \\
\text { America }\end{array}$ & Anderberg et al. 33 (S) & EF558616 \\
\hline $\begin{array}{l}\text { Elaeoluma } \\
\text { schomburgkiana (Miq.) } \\
\text { Baill. }\end{array}$ & Amazon & Keel \& Koelho 243 (S) & DQ246679 \\
\hline $\begin{array}{l}\text { Elaeoluma } \\
\text { schomburgkiana (Miq.) } \\
\text { Baill. }\end{array}$ & Amazon & Keel \& Koelho s.n. (S) & MZ470006 \\
\hline $\begin{array}{l}\text { Englerophytum } \\
\text { magalismontanum } \\
\text { (Sond.) T.D. Penn. }\end{array}$ & Africa & $\begin{array}{l}\text { Swenson \& Karis } 631 \\
\text { (S) }\end{array}$ & DQ246680 \\
\hline $\begin{array}{l}\text { Englerophytum natalense } \\
\text { (Sond.) T.D. Penn. }\end{array}$ & Africa & Kayombo 3483 (S) & AY552150 \\
\hline $\begin{array}{l}\text { Manilkara bidentata (A. } \\
\text { DC.) A. Chev }\end{array}$ & $\begin{array}{l}\text { Magdalena } \\
\text { Valley }\end{array}$ & Serrano 28 (UDBC) & MZ329856 \\
\hline $\begin{array}{l}\text { Manilkara bidentata (A. } \\
\text { DC.) A. Chev }\end{array}$ & Chocó & Serrano 294 (UDBC) & MZ329857 \\
\hline $\begin{array}{l}\text { Manilkara bidentata (A. } \\
\text { DC.) A. Chev }\end{array}$ & Chocó & Serrano 334 (UDBC) & MZ329858 \\
\hline $\begin{array}{l}\text { Manilkara bidentata (A. } \\
\text { DC.) A. Chev }\end{array}$ & Chocó & Serrano 245 (UDBC) & MZ329859 \\
\hline $\begin{array}{l}\text { Micropholis (Griseb.) } \\
\quad \text { Pierre }\end{array}$ & Amazon & Alvarez $737^{\mathrm{a}}$ & MF574537 \\
\hline $\begin{array}{l}\text { Micropholis (Griseb.) } \\
\quad \text { Pierre }\end{array}$ & Amazon & Alvarez $799^{\mathrm{a}}$ & MF574538 \\
\hline $\begin{array}{l}\text { Micropholis (Griseb.) } \\
\quad \text { Pierre }\end{array}$ & Amazon & Sanchez et al. $22^{\text {a }}$ & MF574539 \\
\hline $\begin{array}{l}\text { Micropholis (Griseb.) } \\
\quad \text { Pierre }\end{array}$ & Amazon & $\begin{array}{l}\text { Avila et al. } 2733 \\
\text { (UDBC) }\end{array}$ & MF574540 \\
\hline $\begin{array}{l}\text { Micropholis (Griseb.) } \\
\quad \text { Pierre }\end{array}$ & Amazon & Garcia 8618 (E) & MZ470022 \\
\hline Micropholis & & & \\
\hline $\begin{array}{l}\text { brochidodroma T.D. } \\
\text { Penn. }\end{array}$ & Amazon & JR-JH-1 ${ }^{\text {a }}$ & MF574503 \\
\hline $\begin{array}{l}\text { Micropholis } \\
\quad \text { casiquiarensis Aubrév. }\end{array}$ & Amazon & $\begin{array}{l}\text { Richardson et al. } 340 \\
\text { (E) }\end{array}$ & MF574504 \\
\hline $\begin{array}{l}\text { Micropholis cayennensis } \\
\text { T.D. Penn. }\end{array}$ & Guyanas & $\begin{array}{l}\text { Baraloto, Engel \& Riera } \\
\text { s.n. (CAY) }\end{array}$ & FJ037875.2 \\
\hline $\begin{array}{l}\text { Micropholis } \\
\quad \text { crassipedicellata (Mart. } \\
\text { \& Eichler ex Miq.) } \\
\text { Pierre }\end{array}$ & $\begin{array}{l}\text { Atlantic } \\
\text { Forest }\end{array}$ & NH200222.a (CAY) & FJ037881 \\
\hline $\begin{array}{l}\text { Micropholis } \\
\text { crassipedicellata (Mart. } \\
\text { \& Eichler ex Miq.) } \\
\text { Pierre }\end{array}$ & $\begin{array}{l}\text { Atlantic } \\
\text { Forest }\end{array}$ & C.V. 171 (CEPEC) & JQ434164.1 \\
\hline $\begin{array}{l}\text { Micropholis } \\
\text { crassipedicellata (Mart. } \\
\text { \& Eichler ex Miq.) } \\
\text { Pierre }\end{array}$ & $\begin{array}{l}\text { Atlantic } \\
\text { Forest }\end{array}$ & C.V. 181 (CEPEC) & JQ434165.1 \\
\hline $\begin{array}{l}\text { Micropholis crotonoides } \\
\text { (Pierre) Pierre }\end{array}$ & Llanos & Ramirez et al. $50^{\mathrm{a}}$ & MF574506 \\
\hline $\begin{array}{l}\text { Micropholis crotonoides } \\
\text { (Pierre) Pierre }\end{array}$ & Llanos & ESR89 $^{\mathrm{a}}$ & MF574507 \\
\hline $\begin{array}{l}\text { Micropholis crotonoides } \\
\text { (Pierre) Pierre }\end{array}$ & Llanos & $\mathrm{JRM} 24^{\mathrm{a}}$ & MF574508 \\
\hline $\begin{array}{l}\text { Micropholis egensis (A. } \\
\text { DC.) Pierre }\end{array}$ & Amazon & $\begin{array}{l}\text { Dionizia, Coêlho \& } \\
\text { Ernesto } 73(\mathrm{U})\end{array}$ & DQ246681 \\
\hline
\end{tabular}

(continued)

\begin{tabular}{|c|c|c|c|}
\hline Taxon & Locality & Colector & Genebank \\
\hline $\begin{array}{l}\text { Micropholis egensis (A. } \\
\text { DC.) Pierre }\end{array}$ & Guyanas & Baraloto s.n. (CAY) & FJ037876 \\
\hline $\begin{array}{l}\text { Micropholis egensis (A. } \\
\text { DC.) Pierre }\end{array}$ & Amazon & Avila $2746^{\mathrm{a}}$ & MF574512 \\
\hline $\begin{array}{l}\text { Micropholis egensis (A. } \\
\text { DC.) Pierre }\end{array}$ & Amazon & $\begin{array}{l}\text { Betancur, Echeverry, } \\
\text { Kress \& Roesel } 2848 \\
\text { (K) }\end{array}$ & MZ470021 \\
\hline $\begin{array}{l}\text { Micropholis gardneriana } \\
\text { (A. DC.) Pierre }\end{array}$ & $\begin{array}{l}\text { Atlantic } \\
\text { Forest }\end{array}$ & C.V. 177 (CEPEC) & JQ434158.1 \\
\hline $\begin{array}{l}\text { Micropholis gardneriana } \\
\quad \text { (A. DC.) Pierre }\end{array}$ & $\begin{array}{l}\text { Atlantic } \\
\text { Forest }\end{array}$ & C.V. 205 (CEPEC) & JQ434159.1 \\
\hline $\begin{array}{l}\text { Micropholis gardneriana } \\
\quad \text { (A. DC.) Pierre }\end{array}$ & $\begin{array}{l}\text { Atlantic } \\
\text { Forest }\end{array}$ & C.V. 70 (CEPEC) & JQ434160.1 \\
\hline $\begin{array}{l}\text { Micropholis gardneriana } \\
\quad \text { (A. DC.) Pierre }\end{array}$ & $\begin{array}{l}\text { Atlantic } \\
\text { Forest }\end{array}$ & C.V. 215 (CEPEC) & JQ434161.1 \\
\hline $\begin{array}{l}\text { Micropholis guyanensis } \\
\quad \text { (A. DC.) Pierre }\end{array}$ & West Indies & Taylor 11,691 (MO) & DQ246682 \\
\hline $\begin{array}{l}\text { Micropholis guyanensis } \\
\text { (A. DC.) Pierre }\end{array}$ & Guyanas & $\begin{array}{l}\text { Baraloto, Engel \& } \\
\text { Riviera NH200477 } \\
\text { (CAY) }\end{array}$ & FJ037877 \\
\hline $\begin{array}{l}\text { Micropholis guyanensis } \\
\quad \text { (A. DC.) Pierre }\end{array}$ & Guyanas & Baraloto et al. (CAY) & FJ037878.1 \\
\hline $\begin{array}{l}\text { Micropholis guyanensis } \\
\quad \text { (A. DC.) Pierre }\end{array}$ & $\begin{array}{l}\text { Atlantic } \\
\text { Forest }\end{array}$ & C.V. 113 (CEPEC) & JQ434166.1 \\
\hline $\begin{array}{l}\text { Micropholis guyanensis } \\
\quad \text { (A. DC.) Pierre }\end{array}$ & $\begin{array}{l}\text { Atlantic } \\
\text { Forest }\end{array}$ & C.V. 200 (CEPEC) & JQ434167.1 \\
\hline $\begin{array}{l}\text { Micropholis guyanensis } \\
\quad \text { (A. DC.) Pierre }\end{array}$ & $\begin{array}{l}\text { Atlantic } \\
\text { Forest }\end{array}$ & C.V. 24 (CEPEC) & JQ434168.1 \\
\hline $\begin{array}{l}\text { Micropholis guyanensis } \\
\text { (A. DC.) Pierre }\end{array}$ & $\begin{array}{l}\text { Atlantic } \\
\text { Forest }\end{array}$ & ALCB $110909^{\mathrm{a}}$ & KF943856.1 \\
\hline $\begin{array}{l}\text { Micropholis guyanensis } \\
\quad \text { (A. DC.) Pierre }\end{array}$ & Llanos & CR194 & MF574513 \\
\hline $\begin{array}{l}\text { Micropholis guyanensis } \\
\quad \text { (A. DC.) Pierre }\end{array}$ & Llanos & Miranda et al. $93^{\mathrm{a}}$ & MF574514 \\
\hline $\begin{array}{l}\text { Micropholis guyanensis } \\
\quad \text { (A. DC.) Pierre }\end{array}$ & Llanos & Miranda et al. $106^{\mathrm{a}}$ & MF574515 \\
\hline $\begin{array}{l}\text { Micropholis guyanensis } \\
\quad \text { (A. DC.) Pierre }\end{array}$ & Amazon & Avila $2684^{\mathrm{a}}$ & MF574516 \\
\hline $\begin{array}{l}\text { Micropholis guyanensis } \\
\quad \text { (A. DC.) Pierre }\end{array}$ & Amazon & Pineros et al. $14^{\mathrm{a}}$ & MF574517 \\
\hline $\begin{array}{l}\text { Micropholis guyanensis } \\
\quad \text { (A. DC.) Pierre }\end{array}$ & Amazon & $\begin{array}{l}\text { Richardson et al. } 328 \\
\text { (E) }\end{array}$ & MF574518 \\
\hline $\begin{array}{l}\text { Micropholis guyanensis } \\
\quad \text { (A. DC.) Pierre }\end{array}$ & Amazon & Serrano 68 (UDBC) & MZ329979 \\
\hline $\begin{array}{l}\text { Micropholis guyanensis } \\
\quad \text { (A. DC.) Pierre }\end{array}$ & Amazon & Serrano 71 (UDBC) & MZ329980 \\
\hline $\begin{array}{l}\text { Micropholis guyanensis } \\
\quad \text { (A. DC.) Pierre }\end{array}$ & Amazon & Serrano 53 (UDBC) & MZ329981 \\
\hline $\begin{array}{l}\text { Micropholis guyanensis } \\
\quad \text { (A. DC.) Pierre }\end{array}$ & Amazon & Serrano 77 (UDBC) & MZ329985 \\
\hline $\begin{array}{l}\text { Micropholis guyanensis } \\
\quad \text { (A. DC.) Pierre }\end{array}$ & Amazon & Serrano 66 (UDBC) & MZ329986 \\
\hline $\begin{array}{l}\text { Micropholis guyanensis } \\
\quad \text { (A. DC.) Pierre }\end{array}$ & Amazon & Serrano 52 (UDBC) & MZ329987 \\
\hline $\begin{array}{l}\text { Micropholis guyanensis } \\
\text { (A. DC.) Pierre }\end{array}$ & Amazon & S. coll. $5^{\mathrm{a}}$ & MZ468592 \\
\hline $\begin{array}{l}\text { Micropholis guyanensis } \\
\text { (A. DC.) Pierre }\end{array}$ & Amazon & JR-PA-08 ${ }^{a}$ & MZ468593 \\
\hline $\begin{array}{l}\text { Micropholis guyanensis } \\
\quad \text { (A. DC.) Pierre }\end{array}$ & Guyanas & S. coll. $3^{\mathrm{a}}$ & MZ468595 \\
\hline $\begin{array}{l}\text { Micropholis guyanensis } \\
\text { (A. DC.) Pierre }\end{array}$ & Amazon & Garcia $8620(E)$ & MZ470024 \\
\hline $\begin{array}{l}\text { Micropholis guyanensis } \\
\quad \text { (A. DC.) Pierre }\end{array}$ & Amazon & Garcia $8242(E)$ & MZ470025 \\
\hline $\begin{array}{l}\text { Micropholis guyanensis } \\
\quad \text { (A. DC.) Pierre }\end{array}$ & Amazon & Richardson s.n. (E) & MZ470026 \\
\hline $\begin{array}{l}\text { Micropholis guyanensis } \\
\quad \text { (A. DC.) Pierre }\end{array}$ & Guyanas & Baraloto 3107 (CAY) & NL110516 \\
\hline \multicolumn{4}{|l|}{ Micropholis } \\
\hline $\begin{array}{l}\text { humboldtiana (Roem. } \\
\text { \& Schult.) T.D. Penn. }\end{array}$ & Amazon & PU72 (COAH) & MF574525 \\
\hline \multicolumn{4}{|l|}{ Micropholis } \\
\hline longipedicellata & Guyanas & Baraloto 3210 (CAY) & FJ037879 \\
\hline & Guyanas & Baraloto 3210a (CAY) & NL110459 \\
\hline
\end{tabular}


(continued)

\begin{tabular}{|c|c|c|c|}
\hline Taxon & Locality & Colector & Genebank \\
\hline $\begin{array}{l}\text { Micropholis } \\
\text { longipedicellata } \\
\text { Aubrév. }\end{array}$ & & & \\
\hline $\begin{array}{c}\text { Micropholis macrophylla } \\
\text { (Krause) T.D. Penn. }\end{array}$ & Amazon & EP102 ${ }^{\mathrm{a}}$ & MF574528.1 \\
\hline $\begin{array}{l}\text { Micropholis madeirensis } \\
\text { (Baehni) Aubrév. }\end{array}$ & Amazon & $\begin{array}{l}\text { Cortes et al. } 2869 \\
\text { (UDBC) }\end{array}$ & MF574529 \\
\hline $\begin{array}{l}\text { Micropholis madeirensis } \\
\text { (Baehni) Aubrév. }\end{array}$ & Amazon & MS $3660^{\mathrm{a}}$ & MF574530 \\
\hline $\begin{array}{l}\text { Micropholis madeirensis } \\
\text { (Baehni) Aubrév. }\end{array}$ & Amazon & $\begin{array}{l}\text { Richardson et al. } 329 \\
\text { (E) }\end{array}$ & MF574531 \\
\hline $\begin{array}{l}\text { Micropholis melinoniana } \\
\text { Pierre }\end{array}$ & Amazon & Alvarez $1999^{\mathrm{a}}$ & MF574532 \\
\hline $\begin{array}{l}\text { Micropholis obscura T.D. } \\
\text { Penn. }\end{array}$ & Guyanas & Baraloto 3218 (CAY) & FJ037880.1 \\
\hline $\begin{array}{l}\text { Micropholis obscura T.D. } \\
\text { Penn. }\end{array}$ & Amazon & S. coll. $4^{\mathrm{a}}$ & MZ468591 \\
\hline $\begin{array}{l}\text { Micropholis obscura T.D. } \\
\text { Penn. }\end{array}$ & Guyanas & Baraloto 3218a (CAY) & NH200217 \\
\hline $\begin{array}{l}\text { Micropholis obscura T.D. } \\
\text { Penn. }\end{array}$ & Guyanas & Baraloto 3217 (CAY) & P00610293 \\
\hline $\begin{array}{l}\text { Micropholis } \\
\quad \text { porphyrocarpa } \\
\text { (Baehni) Monach. }\end{array}$ & Guyanas & Baraloto 3220 (CAY) & FJ037882.1 \\
\hline $\begin{array}{l}\text { Micropholis } \\
\qquad \text { porphyrocarpa } \\
\text { (Baehni) Monach. }\end{array}$ & Guyanas & Baraloto 3219 (CAY) & FJ037883.1 \\
\hline $\begin{array}{l}\text { Micropholis splendens } \\
\text { Gilly ex Aubrév. }\end{array}$ & Amazon & $\begin{array}{l}\text { Avila et al. } 2693 \\
\text { (UDBC) }\end{array}$ & MF574541 \\
\hline $\begin{array}{l}\text { Micropholis trunciflora } \\
\text { Ducke }\end{array}$ & Amazon & Cardenas $12266^{\mathrm{a}}$ & MF574542 \\
\hline $\begin{array}{l}\text { Micropholis venulosa } \\
\quad \text { (Mart. \& Eichler) } \\
\text { Pierre }\end{array}$ & Amazon & Assunção 122 (U) & DQ246683.1 \\
\hline $\begin{array}{l}\text { Micropholis venulosa } \\
\quad \text { (Mart. \& Eichler) } \\
\text { Pierre }\end{array}$ & Guyanas & Baraloto (CAY) & FJ037884.1 \\
\hline $\begin{array}{l}\text { Micropholis venulosa } \\
\text { (Mart. \& Eichler) } \\
\text { Pierre }\end{array}$ & Guyanas & Baraloto (CAY) & FJ037885.1 \\
\hline $\begin{array}{l}\text { Micropholis venulosa } \\
\quad \text { (Mart. \& Eichler) } \\
\text { Pierre }\end{array}$ & Amazon & AD8893 (UDBC) & MF574543 \\
\hline $\begin{array}{l}\text { Micropholis venulosa } \\
\quad \text { (Mart. \& Eichler) } \\
\text { Pierre }\end{array}$ & Amazon & Avila et al. 2704 & MF574549 \\
\hline $\begin{array}{l}\text { Micropholis venulosa } \\
\text { (Mart. \& Eichler) } \\
\text { Pierre }\end{array}$ & Llanos & Sanchez et al. $124^{\mathrm{a}}$ & MZ468594 \\
\hline $\begin{array}{l}\text { Micropholis venulosa } \\
\quad \text { (Mart. \& Eichler) } \\
\text { Pierre }\end{array}$ & Amazon & S. coll. $1^{\mathrm{a}}$ & MZ468596 \\
\hline $\begin{array}{l}\text { Micropholis venulosa } \\
\quad \text { (Mart. \& Eichler) } \\
\text { Pierre }\end{array}$ & Amazon & Garcia 8005 (E) & MZ470020 \\
\hline $\begin{array}{l}\text { Micropholis venulosa } \\
\text { (Mart. \& Eichler) } \\
\text { Pierre }\end{array}$ & Amazon & Garcia $8238(E)$ & MZ470023 \\
\hline $\begin{array}{l}\text { Micropholis venulosa } \\
\text { (Mart. \& Eichler) } \\
\text { Pierre }\end{array}$ & Guyanas & Baraloto 3128 (CAY) & NL110260 \\
\hline $\begin{array}{l}\text { Micropholis venulosa } \\
\quad \text { (Mart. \& Eichler) } \\
\text { Pierre }\end{array}$ & Guyanas & Baraloto 3114 (CAY) & NL110297 \\
\hline $\begin{array}{l}\text { Omphalocarpum } \\
\text { pachysteloides Mildbr. } \\
\text { ex Hutch. \& Dalziel }\end{array}$ & Africa & Jongkind 2351 (WAG) & AY552151 \\
\hline $\begin{array}{l}\text { Omphalocarpum } \\
\text { strombocarpum Y.B. } \\
\text { Harv. \& Lovett }\end{array}$ & Africa & $\begin{array}{l}\text { Frimodt-Møller et al. } \\
538 \text { (C) }\end{array}$ & DQ246685 \\
\hline $\begin{array}{l}\text { Pouteria adolfi-friedericii } \\
\text { (Engl.) A. Meeuse }\end{array}$ & Africa & Friis et al. 3502 (UPS) & AY552115 \\
\hline $\begin{array}{l}\text { Pouteria alnifolia (Baker) } \\
\quad \text { Roberty }\end{array}$ & Africa & $\begin{array}{l}\text { Jongkind \& Noyes } \\
1322(\mathrm{MO})\end{array}$ & DQ246686 \\
\hline $\begin{array}{l}\text { Pouteria altissima (A. } \\
\text { Chev.) Baehni }\end{array}$ & Africa & Friis et al. 4145 (UPS) & DQ246687 \\
\hline
\end{tabular}

(continued)

\begin{tabular}{|c|c|c|c|}
\hline Taxon & Locality & Colector & Genebank \\
\hline $\begin{array}{l}\text { Pouteria ambelaniifolia } \\
\text { (Sandwith) T.D. Penn. }\end{array}$ & Guyanas & Baraloto s.n. (CAY) & FJ037886 \\
\hline Pouteria Aubl. & Chocó & Serrano 244 (UDBC) & MZ329862 \\
\hline Pouteria Aubl. & Chocó & Serrano 306 (UDBC) & MZ329864 \\
\hline Pouteria Aubl. & Chocó & Serrano 246 (UDBC) & MZ329865 \\
\hline Pouteria Aubl. & Chocó & Serrano 247 (UDBC) & MZ329866 \\
\hline Pouteria Aubl. & Chocó & Serrano 301 (UDBC) & MZ329871 \\
\hline Pouteria Aubl. & Chocó & Serrano 338 (UDBC) & MZ329877 \\
\hline Pouteria Aubl. & Chocó & Serrano 267 (UDBC) & MZ329893 \\
\hline Pouteria Aubl. & Amazon & Serrano 54 (UDBC) & MZ329903 \\
\hline Pouteria Aubl. & Chocó & Serrano 312 (UDBC) & MZ329938 \\
\hline Pouteria Aubl. & Chocó & Serrano 307 (UDBC) & MZ329977 \\
\hline Pouteria Aubl. & Asia & Armstrong $317^{\mathrm{a}}$ & KF686303 \\
\hline Pouteria Aubl. & Guyanas & $\begin{array}{l}\text { Sabatier \& Molino } \\
\text { Q423 }\end{array}$ & MZ468562 \\
\hline Pouteria Aubl. & Guyanas & $\begin{array}{l}\text { Sabatier \& Molino } 383 \\
\text { (K) }\end{array}$ & MZ468564 \\
\hline Pouteria Aubl. & Guyanas & $\begin{array}{l}\text { Sabatier \& Molino } 213 \\
\text { (P) }\end{array}$ & MZ468565 \\
\hline Pouteria Aubl. & Guyanas & $\begin{array}{l}\text { Sabatier \& Molino } 989 \\
\text { (K) }\end{array}$ & MZ468567 \\
\hline Pouteria Aubl. & Guyanas & $\begin{array}{l}\text { Sabatier \& Molino } \\
\text { V819 }\end{array}$ & MZ468569 \\
\hline Pouteria Aubl. & Amazon & S. coll. $8^{\mathrm{a}}$ & MZ468572 \\
\hline Pouteria Aubl. & $\begin{array}{l}\text { Central } \\
\text { America }\end{array}$ & Anderberg et al. 51 (S) & MZ469959 \\
\hline Pouteria Aubl. & Amazon & Garcia $8777(E)$ & MZ469963 \\
\hline Pouteria Aubl. & Amazon & Garcia $8632(\mathrm{E})$ & MZ469965 \\
\hline Pouteria Aubl. & Amazon & Garcia $8641(\mathrm{E})$ & MZ469972 \\
\hline Pouteria Aubl. & $\begin{array}{l}\text { Central } \\
\text { America }\end{array}$ & Anderberg et al. 60 (S) & MZ469996 \\
\hline Pouteria Aubl. & Amazon & Garcia $8623(\mathrm{E})$ & MZ470019 \\
\hline $\begin{array}{l}\text { Pouteria aubrevillei } \\
\text { Bernardi }\end{array}$ & Amazon & $\begin{array}{l}\text { Richardson et al. } 327 \\
\text { (E) }\end{array}$ & MZ469969 \\
\hline $\begin{array}{l}\text { Pouteria baehniana } \\
\quad \text { Monach }\end{array}$ & Amazon & Serrano 144 (UDBC) & MZ329860 \\
\hline $\begin{array}{l}\text { Pouteria baehniana } \\
\quad \text { Monach }\end{array}$ & Amazon & Serrano 116 (UDBC) & MZ329861 \\
\hline $\begin{array}{l}\text { Pouteria bangii (Rusby) } \\
\text { T.D. Penn. }\end{array}$ & Catatumbo & Serrano 198 (UDBC) & MZ329932 \\
\hline $\begin{array}{l}\text { Pouteria baueri } \\
\quad \text { (Montrouz.) Baehni }\end{array}$ & Australia & Munzinger 340 & AY552113 \\
\hline $\begin{array}{l}\text { Pouteria benai (Aubrév. } \\
\text { \& Pellegr.) T.D. Penn. }\end{array}$ & Guyanas & Mori et al. 27,353 (K) & ED902434 \\
\hline $\begin{array}{l}\text { Pouteria bilocularis (H.J. } \\
\text { P. Winkl.) Baehni }\end{array}$ & Amazon & $\begin{array}{l}\text { Richardson et al. } 345 \\
\text { (E) }\end{array}$ & ED090242 \\
\hline $\begin{array}{l}\text { Pouteria bilocularis (H.J. } \\
\text { P. Winkl.) Baehni }\end{array}$ & Amazon & Garcia 8763 (E) & MZ469995 \\
\hline $\begin{array}{l}\text { Pouteria bracteata T.D. } \\
\text { Penn. }\end{array}$ & Chocó & Serrano 298 (UDBC) & MZ329925 \\
\hline $\begin{array}{l}\text { Pouteria bracteata T.D. } \\
\text { Penn. }\end{array}$ & Chocó & Palacios 13,699 (K) & MZ469974 \\
\hline $\begin{array}{l}\text { Pouteria caimito (Ruiz \& } \\
\text { Pav.) Radlk. }\end{array}$ & Chocó & Serrano 318 (UDBC) & MZ329954 \\
\hline $\begin{array}{l}\text { Pouteria caimito (Ruiz \& } \\
\text { Pav.) Radlk. }\end{array}$ & Chocó & Serrano 311 (UDBC) & MZ329955 \\
\hline $\begin{array}{l}\text { Pouteria caimito (Ruiz \& } \\
\text { Pav.) Radlk. }\end{array}$ & Chocó & Serrano 305 (UDBC) & MZ329956 \\
\hline $\begin{array}{l}\text { Pouteria caimito (Ruiz \& } \\
\text { Pav.) Radlk. }\end{array}$ & Chocó & Serrano 299 (UDBC) & MZ329957 \\
\hline $\begin{array}{l}\text { Pouteria caimito (Ruiz \& } \\
\text { Pav.) Radlk. }\end{array}$ & Chocó & $\begin{array}{l}\text { Richardson et al. } 388 \\
\text { (E) }\end{array}$ & MZ469985 \\
\hline $\begin{array}{l}\text { Pouteria caimito (Ruiz \& } \\
\text { Pav.) Radlk. }\end{array}$ & Chocó & $\begin{array}{l}\text { Richardson et al. } 391 \\
\text { (E) }\end{array}$ & MZ469986 \\
\hline $\begin{array}{l}\text { Pouteria caimito (Ruiz \& } \\
\text { Pav.) Radlk. }\end{array}$ & Chocó & $\begin{array}{l}\text { Richardson et al. } 386 \\
\text { (E) }\end{array}$ & MZ469994 \\
\hline $\begin{array}{l}\text { Pouteria caimito (Ruiz \& } \\
\text { Pav.) Radlk. }\end{array}$ & Chocó & $\begin{array}{l}\text { Richardson et al. } 387 \\
\text { (E) }\end{array}$ & MZ469997 \\
\hline $\begin{array}{l}\text { Pouteria caimito (Ruiz \& } \\
\text { Pav.) Radlk. }\end{array}$ & Chocó & $\begin{array}{l}\text { Richardson et al. } 377 \\
\text { (E) }\end{array}$ & MZ469998 \\
\hline $\begin{array}{l}\text { Pouteria caimito (Ruiz \& } \\
\text { Pav.) Radlk. }\end{array}$ & Chocó & $\begin{array}{l}\text { Richardson et al. } 390 \\
\text { (E) }\end{array}$ & MZ469999 \\
\hline $\begin{array}{l}\text { Pouteria campechiana } \\
\text { (Kunth) Baehni }\end{array}$ & West Indies & Wang W00798 (HAST) & DQ246688 \\
\hline $\begin{array}{l}\text { Pouteria collina (Little) T. } \\
\quad \text { D. Penn. }\end{array}$ & Chocó & Serrano 258 (UDBC) & MZ329948 \\
\hline
\end{tabular}


(continued)

\begin{tabular}{|c|c|c|c|}
\hline Taxon & Locality & Colector & Genebank \\
\hline $\begin{array}{l}\text { Pouteria collina (Little) T. } \\
\text { D. Penn. }\end{array}$ & Chocó & Serrano 263 (UDBC) & MZ329949 \\
\hline $\begin{array}{l}\text { Pouteria collina (Little) T. } \\
\text { D. Penn. }\end{array}$ & Chocó & $\begin{array}{l}\text { Gentry, } \\
\text { Faber-Langendoen \& } \\
\text { Echevarria } 62,908(\mathrm{~K})\end{array}$ & MZ469973 \\
\hline $\begin{array}{l}\text { Pouteria coriacea (Pierre) } \\
\quad \text { Pierre }\end{array}$ & Macarena & Serrano 165 (UDBC) & MZ329926 \\
\hline $\begin{array}{l}\text { Pouteria coriacea (Pierre) } \\
\quad \text { Pierre }\end{array}$ & Macarena & Serrano 174 (UDBC) & MZ329927 \\
\hline $\begin{array}{l}\text { Pouteria coriacea (Pierre) } \\
\quad \text { Pierre }\end{array}$ & Macarena & Serrano 168 (UDBC) & MZ329928 \\
\hline $\begin{array}{l}\text { Pouteria coriacea (Pierre) } \\
\quad \text { Pierre }\end{array}$ & Macarena & Serrano 173 (UDBC) & MZ329929 \\
\hline $\begin{array}{l}\text { Pouteria coriacea (Pierre) } \\
\text { Pierre }\end{array}$ & Macarena & Serrano 169 (UDBC) & MZ329930 \\
\hline $\begin{array}{l}\text { Pouteria coriacea (Pierre) } \\
\text { Pierre }\end{array}$ & Macarena & Serrano 164 (UDBC) & MZ329931 \\
\hline $\begin{array}{l}\text { Pouteria coriacea (Pierre) } \\
\quad \text { Pierre }\end{array}$ & Macarena & Serrano nn $365^{\mathrm{a}}$ & MZ468570 \\
\hline $\begin{array}{l}\text { Pouteria coriacea (Pierre) } \\
\quad \text { Pierre }\end{array}$ & Macarena & Serrano nn364 & MZ468571 \\
\hline $\begin{array}{l}\text { Pouteria cuspidata (A. } \\
\text { DC.) Baehni }\end{array}$ & Chocó & Serrano 337 (UDBC) & MZ329906 \\
\hline $\begin{array}{l}\text { Pouteria cuspidata (A. } \\
\text { DC.) Baehni }\end{array}$ & Catatumbo & Serrano 210 (UDBC) & MZ329907 \\
\hline $\begin{array}{l}\text { Pouteria cuspidata (A. } \\
\text { DC.) Baehni }\end{array}$ & Catatumbo & Serrano 211 (UDBC) & MZ329908 \\
\hline $\begin{array}{l}\text { Pouteria cuspidata (A. } \\
\text { DC.) Baehni }\end{array}$ & Catatumbo & Serrano 208 (UDBC) & MZ329909 \\
\hline $\begin{array}{l}\text { Pouteria cuspidata (A. } \\
\text { DC.) Baehni }\end{array}$ & Catatumbo & Serrano 205 (UDBC) & MZ329910 \\
\hline $\begin{array}{l}\text { Pouteria cuspidata (A. } \\
\text { DC.) Baehni }\end{array}$ & Catatumbo & Serrano 224 (UDBC) & MZ329911 \\
\hline $\begin{array}{l}\text { Pouteria cuspidata (A. } \\
\text { DC.) Baehni }\end{array}$ & Catatumbo & Serrano 207 (UDBC) & MZ329912 \\
\hline $\begin{array}{l}\text { Pouteria cuspidata (A. } \\
\text { DC.) Baehni }\end{array}$ & Catatumbo & Serrano 219 (UDBC) & MZ329913 \\
\hline $\begin{array}{l}\text { Pouteria cuspidata (A. } \\
\text { DC.) Baehni }\end{array}$ & Catatumbo & Serrano 215 (UDBC) & MZ329914 \\
\hline $\begin{array}{l}\text { Pouteria cuspidata (A. } \\
\text { DC.) Baehni }\end{array}$ & Catatumbo & Serrano 212 (UDBC) & MZ329915 \\
\hline $\begin{array}{l}\text { Pouteria cuspidata (A. } \\
\text { DC.) Baehni }\end{array}$ & Chocó & Serrano 242 (UDBC) & MZ329916 \\
\hline $\begin{array}{l}\text { Pouteria cuspidata (A. } \\
\text { DC.) Baehni }\end{array}$ & Catatumbo & Serrano 203 (UDBC) & MZ329917 \\
\hline $\begin{array}{l}\text { Pouteria cuspidata (A. } \\
\text { DC.) Baehni }\end{array}$ & Catatumbo & Serrano 209 (UDBC) & MZ329918 \\
\hline $\begin{array}{l}\text { Pouteria cuspidata (A. } \\
\text { DC.) Baehni }\end{array}$ & Catatumbo & Serrano 204 (UDBC) & MZ329919 \\
\hline $\begin{array}{l}\text { Pouteria cuspidata (A. } \\
\text { DC.) Baehni }\end{array}$ & Catatumbo & Serrano 193 (UDBC) & MZ329920 \\
\hline $\begin{array}{l}\text { Pouteria cuspidata (A. } \\
\text { DC.) Baehni }\end{array}$ & Catatumbo & Serrano 218 (UDBC) & MZ329921 \\
\hline $\begin{array}{l}\text { Pouteria cuspidata (A. } \\
\text { DC.) Baehni }\end{array}$ & Catatumbo & Serrano 185 (UDBC) & MZ329922 \\
\hline $\begin{array}{l}\text { Pouteria cuspidata (A. } \\
\text { DC.) Baehni }\end{array}$ & Catatumbo & Serrano 221 (UDBC) & MZ329923 \\
\hline $\begin{array}{l}\text { Pouteria cuspidata (A. } \\
\text { DC.) Baehni }\end{array}$ & Chocó & Serrano 336 (UDBC) & MZ329924 \\
\hline $\begin{array}{l}\text { Pouteria cuspidata (A. } \\
\text { DC.) Baehni }\end{array}$ & Chocó & Serrano 268 (UDBC) & MZ329933 \\
\hline $\begin{array}{l}\text { Pouteria cuspidata (A. } \\
\text { DC.) Baehni }\end{array}$ & Chocó & Serrano 335 (UDBC) & MZ329934 \\
\hline $\begin{array}{l}\text { Pouteria cuspidata (A. } \\
\text { DC.) Baehni }\end{array}$ & Chocó & Serrano 285 (UDBC) & MZ329935 \\
\hline $\begin{array}{l}\text { Pouteria cuspidata (A. } \\
\text { DC.) Baehni }\end{array}$ & Chocó & Serrano 269 (UDBC) & MZ329936 \\
\hline $\begin{array}{l}\text { Pouteria cuspidata (A. } \\
\text { DC.) Baehni }\end{array}$ & Chocó & Serrano 249 (UDBC) & MZ329937 \\
\hline $\begin{array}{l}\text { Pouteria cuspidata (A. } \\
\text { DC.) Baehni }\end{array}$ & Chocó & Serrano 321 (UDBC) & MZ329939 \\
\hline $\begin{array}{l}\text { Pouteria cuspidata (A. } \\
\text { DC.) Baehni }\end{array}$ & Macarena & Serrano 161 (UDBC) & MZ329940 \\
\hline \multirow[t]{2}{*}{$\begin{array}{l}\text { Pouteria cuspidata (A. } \\
\text { DC.) Baehni }\end{array}$} & Macarena & Serrano 159 (UDBC) & MZ329941 \\
\hline & Macarena & Serrano 167 (UDBC) & MZ329942 \\
\hline
\end{tabular}

(continued)

\begin{tabular}{|c|c|c|c|}
\hline Taxon & Locality & Colector & Genebank \\
\hline \multicolumn{4}{|l|}{$\begin{array}{l}\text { Pouteria cuspidata (A. } \\
\text { DC.) Baehni }\end{array}$} \\
\hline $\begin{array}{l}\text { Pouteria cuspidata (A. } \\
\text { DC.) Baehni }\end{array}$ & Macarena & Serrano 166 (UDBC) & MZ329943 \\
\hline $\begin{array}{l}\text { Pouteria cuspidata (A. } \\
\text { DC.) Baehni }\end{array}$ & Macarena & Serrano 160 (UDBC) & MZ329944 \\
\hline $\begin{array}{l}\text { Pouteria cuspidata (A. } \\
\text { DC.) Baehni }\end{array}$ & Macarena & Serrano 156 (UDBC) & MZ329945 \\
\hline $\begin{array}{l}\text { Pouteria cuspidata (A. } \\
\text { DC.) Baehni }\end{array}$ & Macarena & Serrano 157 (UDBC) & MZ329946 \\
\hline $\begin{array}{l}\text { Pouteria cuspidata (A. } \\
\text { DC.) Baehni }\end{array}$ & Macarena & Serrano 162 (UDBC) & MZ329947 \\
\hline $\begin{array}{l}\text { Pouteria cuspidata (A. } \\
\text { DC.) Baehni }\end{array}$ & Catatumbo & Serrano nn $363^{\mathrm{a}}$ & MZ329982 \\
\hline $\begin{array}{l}\text { Pouteria cuspidata (A. } \\
\text { DC.) Baehni }\end{array}$ & Chocó & Serrano 284 (UDBC) & MZ329983 \\
\hline $\begin{array}{l}\text { Pouteria cuspidata (A. } \\
\text { DC.) Baehni }\end{array}$ & Chocó & Serrano 241 (UDBC) & MZ329984 \\
\hline $\begin{array}{l}\text { Pouteria cuspidata (A. } \\
\text { DC.) Baehni }\end{array}$ & Catatumbo & Serrano 158 (UDBC) & MZ468556 \\
\hline $\begin{array}{l}\text { Pouteria cuspidata (A. } \\
\text { DC.) Baehni }\end{array}$ & Catatumbo & Serrano 175 (UDBC) & MZ468573 \\
\hline $\begin{array}{l}\text { Pouteria cuspidata (A. } \\
\text { DC.) Baehni }\end{array}$ & Catatumbo & Serrano nn $368^{\mathrm{a}}$ & MZ468574 \\
\hline $\begin{array}{l}\text { Pouteria cuspidata (A. } \\
\text { DC.) Baehni }\end{array}$ & Catatumbo & Serrano nn $369^{a}$ & MZ468575 \\
\hline $\begin{array}{l}\text { Pouteria cuspidata (A. } \\
\text { DC.) Baehni }\end{array}$ & Catatumbo & Serrano nn366 & MZ468576 \\
\hline $\begin{array}{l}\text { Pouteria cuspidata (A. } \\
\text { DC.) Baehni }\end{array}$ & Catatumbo & Serrano nn $370^{\mathrm{a}}$ & MZ468577 \\
\hline $\begin{array}{l}\text { Pouteria cuspidata (A. } \\
\text { DC.) Baehni }\end{array}$ & Catatumbo & Serrano 176 (UDBC) & MZ468578 \\
\hline $\begin{array}{l}\text { Pouteria cuspidata (A. } \\
\text { DC.) Baehni }\end{array}$ & Amazon & $\begin{array}{l}\text { Richardson et al. } 337 \\
\text { (E) }\end{array}$ & MZ469976 \\
\hline $\begin{array}{l}\text { Pouteria cuspidata (A. } \\
\text { DC.) Baehni }\end{array}$ & Amazon & Garcia 8624 (E) & MZ469977 \\
\hline $\begin{array}{l}\text { Pouteria cuspidata (A. } \\
\text { DC.) Baehni }\end{array}$ & Amazon & Garcia $8769(E)$ & MZ469978 \\
\hline $\begin{array}{l}\text { Pouteria decorticans T.D. } \\
\text { Penn. }\end{array}$ & Guyanas & $\begin{array}{l}\text { Baraloto, Engel, Chave } \\
\text { \& Riera s.n. (CAY) }\end{array}$ & FJ037887 \\
\hline $\begin{array}{l}\text { Pouteria deliciosa T.D. } \\
\text { Penn. }\end{array}$ & Guyanas & Sabatier \& Molino $903^{\mathrm{a}}$ & MZ468563 \\
\hline $\begin{array}{l}\text { Pouteria dominigensis (C. } \\
\text { F. Gaertn.) Baehni }\end{array}$ & West Indies & $\begin{array}{l}\text { Gutiérrez \& Nilsson } 13 \\
\text { (S) }\end{array}$ & AY552106 \\
\hline $\begin{array}{l}\text { Pouteria dominigensis (C. } \\
\text { F. Gaertn.) Baehni }\end{array}$ & $\begin{array}{l}\text { Magdalena } \\
\text { Valley }\end{array}$ & Serrano 351 (UDBC) & MZ329876 \\
\hline $\begin{array}{l}\text { Pouteria eugeniifolia } \\
\text { (Pierre) Baehni }\end{array}$ & Guyanas & Baraloto 3136 (CAY) & FJ037888 \\
\hline $\begin{array}{l}\text { Pouteria eugeniifolia } \\
\text { (Pierre) Baehni }\end{array}$ & Guyanas & $\begin{array}{l}\text { Baraloto, Engel, Chave } \\
\text { \& Riera s.n. (CAY) }\end{array}$ & FJ037889.1 \\
\hline Pouteria filipes Eyma & Amazon & Serrano 140 (UDBC) & MZ329884 \\
\hline Pouteria filipes Eyma & Amazon & Serrano 73 (UDBC) & MZ329885 \\
\hline $\begin{array}{l}\text { Pouteria gardneriana (A. } \\
\text { DC.) Radlk. }\end{array}$ & Argentina & Schwarz 8216 (UPS) & DQ246689 \\
\hline Pouteria gongrijpii Eyma & Guyanas & Baraloto 3087 (CAY) & FJ037890 \\
\hline Pouteria gongrijpii Eyma & Guyanas & Baraloto 3098 (CAY) & FJ037891 \\
\hline Pouteria grandis Eyma & Guyanas & Sabatier \& Molino $995^{\mathrm{a}}$ & MZ469960 \\
\hline Pouteria guianensis Aubl. & Guyanas & Poncy $1745(\mathrm{P})$ & DQ246690 \\
\hline Pouteria guianensis Aubl. & Guyanas & $\begin{array}{l}\text { Baraloto \& Engel s.n. } \\
\text { (CAY) }\end{array}$ & FJ037892 \\
\hline Pouteria guianensis Aubl. & Guyanas & $\begin{array}{l}\text { Baraloto, Engel, Chave } \\
\& \text { Riera } 3102 \text { (CAY) }\end{array}$ & FJ037893 \\
\hline Pouteria guianensis Aubl. & Amazon & $\begin{array}{l}\text { Richardson et al. } 332 \\
\text { (E) }\end{array}$ & MZ469954 \\
\hline Pouteria guianensis Aubl. & Amazon & $\begin{array}{l}\text { Richardson et al. } 342 \\
\text { (E) }\end{array}$ & MZ469987 \\
\hline Pouteria guianensis Aubl. & Amazon & $\begin{array}{l}\text { Richardson et al. } 309 \\
\text { (E) }\end{array}$ & MZ469988 \\
\hline Pouteria guianensis Aubl. & Amazon & $\begin{array}{l}\text { Richardson et al. } 323 \\
\text { (E) }\end{array}$ & MZ469989 \\
\hline Pouteria guianensis Aubl. & Amazon & Garcia 8631 (E) & MZ469990 \\
\hline Pouteria guianensis Aubl. & Amazon & $\begin{array}{l}\text { Richardson et al. } 351 \\
\text { (E) }\end{array}$ & MZ469991 \\
\hline Pouteria guianensis Aubl. & Amazon & $\begin{array}{l}\text { Richardson et al. } 333 \\
\text { (E) }\end{array}$ & MZ469992 \\
\hline Pouteria hispida Eyma & Guyanas & Mori et al. 25,432 (NY) & DQ246691 \\
\hline
\end{tabular}


(continued)

\begin{tabular}{|c|c|c|c|}
\hline Taxon & Locality & Colector & Genebank \\
\hline $\begin{array}{l}\text { Pouteria laevigata } \\
\text { (Mart.) Radlk. }\end{array}$ & Chocó & Serrano 339 (UDBC) & MZ329960 \\
\hline $\begin{array}{l}\text { Pouteria laevigata } \\
\text { (Mart.) Radlk. }\end{array}$ & $\begin{array}{l}\text { Central } \\
\text { America }\end{array}$ & Anderberg et al. 49 (S) & MZ470001 \\
\hline $\begin{array}{l}\text { Pouteria lecythidicarpa P. } \\
\text { E. Sánchez \& Poveda }\end{array}$ & $\begin{array}{l}\text { Magdalena } \\
\text { Valley }\end{array}$ & Serrano 24 (UDBC) & MZ329878 \\
\hline $\begin{array}{l}\text { Pouteria lecythidicarpa P. } \\
\text { E. Sánchez \& Poveda }\end{array}$ & $\begin{array}{l}\text { Magdalena } \\
\text { Valley }\end{array}$ & Serrano $352^{\mathrm{a}}$ & MZ329879 \\
\hline $\begin{array}{l}\text { Pouteria lecythidicarpa P. } \\
\text { E. Sánchez \& Poveda }\end{array}$ & $\begin{array}{l}\text { Magdalena } \\
\text { Valley }\end{array}$ & Serrano 18 (UDBC) & MZ329880 \\
\hline $\begin{array}{l}\text { Pouteria lecythidicarpa P. } \\
\text { E. Sánchez \& Poveda }\end{array}$ & $\begin{array}{l}\text { Magdalena } \\
\text { Valley }\end{array}$ & Serrano 44 (UDBC) & MZ329881 \\
\hline $\begin{array}{l}\text { Pouteria lecythidicarpa P. } \\
\text { E. Sánchez \& Poveda }\end{array}$ & $\begin{array}{l}\text { Central } \\
\text { America }\end{array}$ & Anderberg et al. 34 (S) & MZ469961 \\
\hline $\begin{array}{l}\text { Pouteria lucumifolia } \\
\quad \text { (Reissek ex Maxim.) T. } \\
\text { D. Penn. }\end{array}$ & Amazon & $\begin{array}{l}\text { Richardson et al. } 306 \\
\text { (E) }\end{array}$ & MZ469975 \\
\hline $\begin{array}{l}\text { Pouteria macrocarpa } \\
\text { (Mart.) D. Dietr. }\end{array}$ & Guyanas & $\begin{array}{l}\text { Baraloto, Engel, Chave } \\
\text { \& Riera } 3095 \text { (CAY) }\end{array}$ & FJ037894 \\
\hline $\begin{array}{l}\text { Pouteria macrophylla } \\
\text { (Lam.) Eyma }\end{array}$ & Amazon & Seidel et al. 5905 (K) & DQ246692 \\
\hline $\begin{array}{l}\text { Pouteria multiflora (A. } \\
\text { DC.) Eyma }\end{array}$ & Chocó & Villa \& Rivaz 257 (BM) & DQ246693 \\
\hline $\begin{array}{l}\text { Pouteria oblanceolata } \\
\quad \text { Pires }\end{array}$ & Amazon & $\begin{array}{l}\text { Richardson et al. } 307 \\
\text { (E) }\end{array}$ & MZ470002 \\
\hline $\begin{array}{l}\text { Pouteria oblanceolata } \\
\quad \text { Pires }\end{array}$ & Amazon & Garcia 8625 (E) & MZ470003 \\
\hline $\begin{array}{l}\text { Pouteria oblanceolata } \\
\quad \text { Pires }\end{array}$ & Amazon & Garcia 8635 (E) & MZ470004 \\
\hline $\begin{array}{l}\text { Pouteria oblanceolata } \\
\quad \text { Pires }\end{array}$ & Amazon & Garcia 8634 (E) & MZ470005 \\
\hline $\begin{array}{l}\text { Pouteria platyphylla (A. } \\
\text { C. Sm.) Baehni }\end{array}$ & Chocó & Serrano 303 (UDBC) & MZ329870 \\
\hline $\begin{array}{l}\text { Pouteria platyphylla (A. } \\
\text { C. Sm.) Baehni }\end{array}$ & $\begin{array}{l}\text { Magdalena } \\
\text { Valley }\end{array}$ & Serrano $359^{\mathrm{a}}$ & MZ329892 \\
\hline $\begin{array}{l}\text { Pouteria platyphylla (A. } \\
\text { C. Sm.) Baehni }\end{array}$ & Catatumbo & Serrano 223 (UDBC) & MZ329894 \\
\hline $\begin{array}{l}\text { Pouteria platyphylla (A. } \\
\text { C. Sm.) Baehni }\end{array}$ & $\begin{array}{l}\text { Magdalena } \\
\text { Valley }\end{array}$ & Serrano $348^{\mathrm{a}}$ & MZ329895 \\
\hline $\begin{array}{l}\text { Pouteria platyphylla (A. } \\
\text { C. Sm.) Baehni }\end{array}$ & $\begin{array}{l}\text { Magdalena } \\
\text { Valley }\end{array}$ & Serrano $350^{\mathrm{a}}$ & MZ329896 \\
\hline $\begin{array}{l}\text { Pouteria platyphylla (A. } \\
\text { C. Sm.) Baehni }\end{array}$ & Amazon & Serrano 55 (UDBC) & MZ329897 \\
\hline $\begin{array}{l}\text { Pouteria platyphylla (A. } \\
\text { C. Sm.) Baehni }\end{array}$ & Amazon & $\begin{array}{l}\text { Richardson et al. } 313 \\
\text { (E) }\end{array}$ & MZ469964 \\
\hline $\begin{array}{l}\text { Pouteria procera (Mart.) } \\
\text { K. Hammer }\end{array}$ & Amazon & $\begin{array}{l}\text { Richardson et al. } 339 \\
\text { (E) }\end{array}$ & MZ469966 \\
\hline $\begin{array}{l}\text { Pouteria pubescens } \\
\text { (Aubrév. \& Pellegr.) T. } \\
\text { D. Penn. }\end{array}$ & Amazon & S. coll. $6^{\mathrm{a}}$ & MZ468566 \\
\hline $\begin{array}{l}\text { Pouteria pubescens } \\
\text { (Aubrév. \& Pellegr.) T. } \\
\text { D. Penn. }\end{array}$ & Amazon & Garcia 8636 (E) & MZ469962 \\
\hline $\begin{array}{l}\text { Pouteria putamen-ovi } T \text {. } \\
\text { D. Penn. }\end{array}$ & Amazon & S. coll. $7^{\mathrm{a}}$ & MZ468568 \\
\hline $\begin{array}{l}\text { Pouteria putamen-ovi T. } \\
\text { D. Penn. }\end{array}$ & Amazon & Garcia 8774 (E) & MZ469970 \\
\hline $\begin{array}{l}\text { Pouteria resinosa T.D. } \\
\text { Penn. }\end{array}$ & Chocó & Serrano 270 (UDBC) & MZ329886 \\
\hline $\begin{array}{l}\text { Pouteria resinosa T.D. } \\
\text { Penn. }\end{array}$ & Chocó & Serrano 259 (UDBC) & MZ329887 \\
\hline $\begin{array}{l}\text { Pouteria resinosa T.D. } \\
\text { Penn. }\end{array}$ & Chocó & Serrano 243 (UDBC) & MZ329889 \\
\hline $\begin{array}{l}\text { Pouteria reticulata } \\
\text { (Engl.) Eyma }\end{array}$ & Amazon & Serrano 72 (UDBC) & MZ329951 \\
\hline $\begin{array}{l}\text { Pouteria reticulata } \\
\text { (Engl.) Eyma }\end{array}$ & $\begin{array}{l}\text { Central } \\
\text { America }\end{array}$ & Anderberg et al. 7 (S) & MZ469993 \\
\hline $\begin{array}{l}\text { Pouteria rostrata (Huber) } \\
\text { Baehni }\end{array}$ & $\begin{array}{l}\text { Magdalena } \\
\text { Valley }\end{array}$ & Serrano 357 (UDBC) & MZ329891 \\
\hline $\begin{array}{l}\text { Pouteria sessilis T.D. } \\
\text { Penn. }\end{array}$ & Amazon & Garcia 8637 (E) & MZ469951 \\
\hline $\begin{array}{l}\text { Pouteria sessilis T.D. } \\
\text { Penn. }\end{array}$ & Amazon & $\begin{array}{l}\text { Richardson et al. } 321 \\
\text { (E) }\end{array}$ & MZ469983 \\
\hline \multirow[t]{2}{*}{$\begin{array}{l}\text { Pouteria subrotata } \\
\text { Cronquist }\end{array}$} & Amazon & Serrano 145 (UDBC) & MZ329967 \\
\hline & Amazon & Serrano 151 (UDBC) & MZ329968 \\
\hline
\end{tabular}

(continued)

\begin{tabular}{|c|c|c|c|}
\hline Taxon & Locality & Colector & Genebank \\
\hline \multicolumn{4}{|l|}{ Pouteria subrotata } \\
\hline $\begin{array}{l}\text { Pouteria subrotata } \\
\text { Cronquist }\end{array}$ & Amazon & Serrano 112 (UDBC) & MZ329969 \\
\hline $\begin{array}{l}\text { Pouteria subrotata } \\
\text { Cronquist }\end{array}$ & $\begin{array}{l}\text { Central } \\
\text { America }\end{array}$ & Anderberg et al. 48 (S) & MZ470000 \\
\hline $\begin{array}{l}\text { Pouteria torta (Mart.) } \\
\text { Radlk. }\end{array}$ & Guyanas & $\begin{array}{l}\text { Baraloto, Engel \& Riera } \\
\text { s.n. (CAY) }\end{array}$ & FJ037895 \\
\hline $\begin{array}{l}\text { Pouteria torta (Mart.) } \\
\text { Radlk. }\end{array}$ & Amazon & Serrano 90 (UDBC) & MZ329863 \\
\hline $\begin{array}{l}\text { Pouteria torta (Mart.) } \\
\text { Radlk. }\end{array}$ & Amazon & Serrano 58 (UDBC) & MZ329883 \\
\hline $\begin{array}{l}\text { Pouteria torta (Mart.) } \\
\text { Radlk. }\end{array}$ & Amazon & Serrano 70 (UDBC) & MZ329898 \\
\hline $\begin{array}{l}\text { Pouteria torta (Mart.) } \\
\text { Radlk. }\end{array}$ & Amazon & Serrano 59 (UDBC) & MZ329899 \\
\hline $\begin{array}{l}\text { Pouteria torta (Mart.) } \\
\text { Radlk. }\end{array}$ & Amazon & Serrano 105 (UDBC) & MZ329900 \\
\hline $\begin{array}{l}\text { Pouteria torta (Mart.) } \\
\text { Radlk. }\end{array}$ & Amazon & Serrano 78 (UDBC) & MZ329901 \\
\hline $\begin{array}{l}\text { Pouteria torta (Mart.) } \\
\text { Radlk. }\end{array}$ & Amazon & Serrano 153 (UDBC) & MZ329961 \\
\hline $\begin{array}{l}\text { Pouteria torta (Mart.) } \\
\text { Radlk. }\end{array}$ & Amazon & Serrano 50 (UDBC) & MZ329962 \\
\hline $\begin{array}{l}\text { Pouteria torta (Mart.) } \\
\text { Radlk. }\end{array}$ & Amazon & Serrano 69 (UDBC) & MZ329978 \\
\hline $\begin{array}{l}\text { Pouteria torta (Mart.) } \\
\text { Radlk. }\end{array}$ & Amazon & Guy $55^{\mathrm{a}}$ & MZ468588 \\
\hline $\begin{array}{l}\text { Pouteria torta (Mart.) } \\
\text { Radlk. }\end{array}$ & Amazon & $\begin{array}{l}\text { Richardson et al. } 338 \\
\text { (E) }\end{array}$ & MZ469967 \\
\hline $\begin{array}{l}\text { Pouteria torta (Mart.) } \\
\text { Radlk. }\end{array}$ & Amazon & Anderberg et al. 47 (S) & MZ469981 \\
\hline $\begin{array}{l}\text { Pouteria torta (Mart.) } \\
\text { Radlk. }\end{array}$ & Amazon & $\begin{array}{l}\text { Richardson et al. } 336 \\
\text { (E) }\end{array}$ & MZ469984 \\
\hline $\begin{array}{l}\text { Pouteria triplarifolia } \\
\text { Standl. \& L.O. Williams } \\
\text { ex T.D. Penn. }\end{array}$ & $\begin{array}{l}\text { Central } \\
\text { America }\end{array}$ & Swenson et al. 738 (S) & MZ469971 \\
\hline $\begin{array}{l}\text { Pouteria venosa (Mart.) } \\
\quad \text { Baehni }\end{array}$ & Amazon & Serrano 149 (UDBC) & MZ329952 \\
\hline $\begin{array}{l}\text { Pouteria venosa (Mart.) } \\
\quad \text { Baehni }\end{array}$ & Amazon & Serrano 76 (UDBC) & MZ329953 \\
\hline $\begin{array}{l}\text { Pouteria vernicosa T.D. } \\
\text { Penn. }\end{array}$ & Chocó & Villa et al. 1304 (BM) & DQ246694 \\
\hline $\begin{array}{l}\text { Pouteria viridis (Pittier) } \\
\text { Cronquist }\end{array}$ & $\begin{array}{l}\text { Central } \\
\text { America }\end{array}$ & $\begin{array}{l}\text { Faber-Langendoen \& } \\
\text { Renteria } 1127(\mathrm{~K})\end{array}$ & MZ468561 \\
\hline $\begin{array}{l}\text { Pradosia atroviolacea } \\
\text { Ducke }\end{array}$ & $\begin{array}{l}\text { Central } \\
\text { America }\end{array}$ & Anderberg et al. 52 (S) & KM042306 \\
\hline $\begin{array}{l}\text { Pradosia atroviolacea } \\
\quad \text { Ducke }\end{array}$ & Chocó & Cuatrecasas 13,988 (K) & KM042310 \\
\hline $\begin{array}{l}\text { Pradosia brevipes (Pierre) } \\
\text { T.D. Penn. }\end{array}$ & Amazon & Lindeman $6743(\mathrm{U})$ & AY552158 \\
\hline $\begin{array}{l}\text { Pradosia cuatrecasasii } \\
\text { (Aubrév.) T.D. Penn. }\end{array}$ & Chocó & $\begin{array}{l}\text { Baraloto, Engel \& Riera } \\
\text { s.n. (CAY) }\end{array}$ & FJ037897 \\
\hline Pradosia Liais & Amazon & Serrano 56 (UDBC) & MZ329872 \\
\hline $\begin{array}{l}\text { Pradosia ptychandra } \\
\text { (Eyma) T.D. Penn. }\end{array}$ & Guyanas & $\begin{array}{l}\text { Baraloto, Engel, Chave } \\
\text { \& Riera } 3111 \text { (CAY) }\end{array}$ & FJ037898 \\
\hline $\begin{array}{l}\text { Pradosia ptychandra } \\
\text { (Eyma) T.D. Penn. }\end{array}$ & Guyanas & $\begin{array}{l}\text { Baraloto, Engel, Chave } \\
\text { \& Riera s.n. (CAY) }\end{array}$ & FJ037899.1 \\
\hline $\begin{array}{l}\text { Pradosia ptychandra } \\
\text { (Eyma) T.D. Penn. }\end{array}$ & Guyanas & $\begin{array}{l}\text { Baraloto, Engel, Chave } \\
\text { \& Riera } 3111 \text { (CAY) }\end{array}$ & MZ468557 \\
\hline $\begin{array}{l}\text { Pradosia schomburgkiana } \\
\text { (A. DC.) Cronquist }\end{array}$ & Amazon & $\begin{array}{l}\text { Ducke Reserve } \\
05-1829(\mathrm{~K})\end{array}$ & AY552156 \\
\hline $\begin{array}{l}\text { Pradosia spinosa Ewango } \\
\quad \text { \& Breteler }\end{array}$ & Africa & Isembe 508 (WAG) & DQ246695 \\
\hline $\begin{array}{l}\text { Pradosia surinamensis } \\
\text { (Eyma) T.D. Penn. }\end{array}$ & Guyanas & Harris $1076(U)$ & AY552157 \\
\hline Sapotaceae Juss. & Guyanas & Guy $37^{\mathrm{a}}$ & FJ037896 \\
\hline Sapotaceae Juss. & Chocó & Serrano 288 (UDBC) & MZ329888 \\
\hline Sapotaceae Juss. & Chocó & Serrano 278 (UDBC) & MZ329890 \\
\hline Sapotaceae Juss. & Chocó & Serrano 315 (UDBC) & MZ329905 \\
\hline Sapotaceae Juss. & $\begin{array}{l}\text { Magdalena } \\
\text { Valley }\end{array}$ & Serrano 38 (UDBC) & MZ468558 \\
\hline Sapotaceae Juss. & $\begin{array}{l}\text { Magdalena } \\
\text { Valley }\end{array}$ & Serrano 39 (UDBC) & MZ468559 \\
\hline Sapotaceae Juss. & Guyanas & Guy $24^{\mathrm{a}}$ & MZ468581 \\
\hline Sapotaceae Juss. & Guyanas & Guy $3^{\mathrm{a}}$ & MZ468582 \\
\hline
\end{tabular}

(continued on next page) 
(continued)

\begin{tabular}{|c|c|c|c|}
\hline Taxon & Locality & Colector & Genebank \\
\hline Sapotaceae Juss. & Guyanas & Guy $76^{a}$ & MZ468583 \\
\hline Sapotaceae Juss. & Guyanas & Guy $13^{\mathrm{a}}$ & MZ468584 \\
\hline Sapotaceae Juss. & Guyanas & Guy $2^{\mathrm{a}}$ & MZ468585 \\
\hline Sapotaceae Juss. & Guyanas & Guy $74^{a}$ & MZ468586 \\
\hline Sapotaceae Juss. & Guyanas & Guy $47^{\mathrm{a}}$ & MZ468587 \\
\hline Sapotaceae Juss. & Guyanas & Guy $32^{\mathrm{a}}$ & MZ468589 \\
\hline $\begin{array}{l}\text { Sarcaulus brasiliensis (A. } \\
\text { DC.) Eyma }\end{array}$ & Amazon & $\begin{array}{l}\text { Paniagua et al. } 4852 \\
\text { (MO) }\end{array}$ & DQ246696 \\
\hline $\begin{array}{l}\text { Sarcaulus brasiliensis (A. } \\
\text { DC.) Eyma }\end{array}$ & $\begin{array}{l}\text { Magdalena } \\
\text { Valley }\end{array}$ & Serrano $355^{\mathrm{a}}$ & MZ329950 \\
\hline $\begin{array}{l}\text { Sarcaulus brasiliensis (A. } \\
\text { DC.) Eyma }\end{array}$ & Amazon & $\begin{array}{l}\text { Richardson et al. } 322 \\
\text { (E) }\end{array}$ & MZ469979 \\
\hline $\begin{array}{l}\text { Sarcosperma laurinum } \\
\text { (Benth.) Hook. } f .\end{array}$ & Asia & Saunders s.n. (S) & AM408055 \\
\hline $\begin{array}{l}\text { Synsepalum dulcificum } \\
\text { (Schumach. \& Thonn.) } \\
\text { Daniell }\end{array}$ & Africa & $\begin{array}{l}\text { Welsing et al. } 24 \\
\text { (WAG) }\end{array}$ & DQ246697 \\
\hline $\begin{array}{l}\text { Synsepalum passargei } \\
\text { (Engl.) T.D. Penn. }\end{array}$ & Africa & Magogo 2452 (UPS) & DQ246698 \\
\hline $\begin{array}{l}\text { Xantolis siamensis } \\
\text { (Fletcher) P. Royen }\end{array}$ & Asia & Smitairi 1 (L) & AY552154.1 \\
\hline
\end{tabular}

\section{Acknowledgements}

We thank Michelle Hart and Laura Forest for laboratory assistance at the Royal Botanic Garden Edinburgh. Fabio Ávila and Doris Huertas are thanked for their assistance at the Herbario Forestal. Terry Pennington and Felix Forest at the Royal Botanic Gardens, Kew are thanked for providing samples of Colombian Sapotaceae and permitting their extraction respectively. Alain Franc supplied samples from French Guiana that were collected by Daniel Sabatier and Jean-François Molino all of whom are thanked for those contributions. Investissement d'Avenir" grants managed by Agence Nationale de la Recherche (CEBA, ref. ANR-10-LABX-25-01) is thanked for their financial to the work carried out by Jérôme Chave's Laboratory. Unpublished data was provided by Dayana Sanchez from the Universidad Distrital Francisco José de Caldas, and by Roosevelt Garcia from the Cornell University. The work was supported by a University of Edinburgh Darwin Trust PhD Scholarship awarded to the first author.

The authors would like to apologise again for any inconvenience caused. 


\section{Update 2 of 2 \\ Global and Planetary Change}

Volume 204, Issue, September 2021, Page

DOI: https://doi.org/10.1016/j.gloplacha.2021.103575 


\title{
Corrigendum to "Andean orogeny and the diversification of lowland neotropical rain forest trees: A case study in Sapotaceae" [Global and Planetary Change 201 (2021) 103481]
}

\author{
Julieth Serrano $^{\text {a, }}$, James E. Richardson ${ }^{\text {b,c }}$, Richard I. Milne ${ }^{\text {d }, ~ G . ~ A r i a d n a ~ M o n d r a g o n ~}{ }^{\text {, }}$ \\ Dairon Cárdenas $^{\mathrm{j}}$, S. Dayana Sanchez ${ }^{\mathrm{k}}$, Rocio Cortés-B ${ }^{\mathrm{k}}$, R. Toby Pennington ${ }^{\text {a,c }}$ \\ ${ }^{a}$ Geography Department, College of Life and Environmental Sciences, University of Exeter, Rennes Drive, Exeter EX4 4RJ, UK \\ ${ }^{\mathrm{b}}$ Facultad de Ciencias Naturales, Universidad del Rosario, Bogotá, DC, Colombia \\ ${ }^{\mathrm{c}}$ Tropical Diversity Section, Royal Botanic Garden Edinburgh, 20a Inverleith Row, Edinburgh EH3 5LR, UK \\ ${ }^{\mathrm{d}}$ Institute of Molecular Plant Sciences, Kings Building, West Mains Road, University of Edinburgh, EH9 3JW, UK \\ e Laboratorio de Botánica y Sistemática, Universidad de los Andes, Apartado Áereo 4976, Bogotá, DC, Colombia \\ ${ }^{\mathrm{f}}$ School of Biological Sciences, University of Reading, Reading RG6 6BX, UK \\ ${ }^{g}$ Department of Population Ecology, Institute of Botany, Academy of Sciences, CZ-25243 Prìhonice, Czech Republic \\ ${ }^{\mathrm{h}}$ Instituto Alexander von Humboldt, Avenida Paseo Bolivar (Circunvalar) 16-20, Bogotá, DC, Colombia \\ ${ }^{\mathrm{i}}$ Laboratoire Evolution et Diversite Biologique UMR 5174, Universite Paul Sabatier/CNRS, 31062 Toulouse, France \\ ${ }^{\mathrm{j}}$ Instituto Amaźonico de Investigaciones Científicas Sinchi, Calle 20 \# 5-44, Bogotá, DC, Colombia \\ ${ }^{\mathrm{k}}$ Herbario Forestal, Universidad Distrital, Carrera 5 Este \# 15-82, Bogotá, DC, Colombia \\ ${ }^{1}$ Jardín Botánico de Cartagena "Guillermo Piñeres", Turbaco, Bolívar, Colombia
} Julie A. Hawkins ${ }^{\text {f }}$, Igor V. Bartish ${ }^{g}$, Mailyn Gonzalez ${ }^{\text {, }}$, Jérôme Chave ${ }^{\mathrm{i}}$, Santiago Madriñán ${ }^{\mathrm{e}, 1}$,

The authors apologise for omitting a full record of GenBank numbers, and a complete list is provided here (Table Appendix 1) together with an update on the Acknowledgements section.-

Appendix 1 List of specimens included in the phylogenetic analyses. Internal transcribed spacers of rDNA (ITS) sequence data was generated. A total of 146 new Chrysophylloideae accessions were collected in the Chocó, Magdalena valley, Catatumbo, Amazon and Macarena lowland rain forests of Colombia. The remaining sequences were generated by Roosevelt Garcia, and obtained from previous phylogenetic studies on Chrysophylloideae (Sánchez-C. et al., 2021; Gonzalez et al., 2009; Swenson et al., 2008), and GenBank.

\begin{tabular}{|c|c|c|c|}
\hline Taxon & Locality & Colector & Genebank \\
\hline Breviea sericea (A. Chev.) Aubrév. \& Pellegr. & Africa & Letouzey $8319(\mathrm{P})$ & DQ246666 \\
\hline Chromolucuma rubriflora Ducke & Central America & Anderberg et al. 20 (S) & EF558614 \\
\hline Chrysophyllum argenteum Jacq. & Chocó & Dick 81 (STRI) & AY635511 \\
\hline Chrysophyllum argenteum Jacq. & Central America & Dick 80 (STRI) & AY635513 \\
\hline Chrysophyllum argenteum Jacq. & Chocó & Dick 85 (STRI) & AY635515 \\
\hline Chrysophyllum argenteum Jacq. & Guianas & Baraloto, Engel \& Riera (CAY) & FJ037865 \\
\hline Chrysophyllum argenteum Jacq. & Central America & Dick 79 (STRI) & JX987536.1 \\
\hline Chrysophyllum argenteum Jacq. & Chocó & Dick 87 (STRI) & JX987537 \\
\hline Chrysophyllum argenteum Jacq. & Chocó & Dick 82 (STRI) & JX987538 \\
\hline Chrysophyllum argenteum Jacq. & Chocó & Serrano 286 (UDBC) & MZ329867 \\
\hline Chrysophyllum argenteum Jacq. & Macarena & Serrano nn367 & MZ329868 \\
\hline Chrysophyllum argenteum Jacq. & Chocó & Serrano 280 (UDBC) & MZ329869 \\
\hline Chrysophyllum argenteum Jacq. & Guianas & Baraloto 3203 (CAY) & FJ037864 \\
\hline Chrysophyllum bangweolense R.E. Fr. & Africa & Malaisse 9600 (WAG) & AY552152.1 \\
\hline Chrysophyllum bicolor Poir. & West Indies & JP57 (DAV) & JF912987 \\
\hline
\end{tabular}

DOI of original article: https://doi.org/10.1016/j.gloplacha.2021.103481.

* Corresponding author.

E-mail address: juliserranoa@yahoo.com (J. Serrano).

https://doi.org/10.1016/j.gloplacha.2021.103575

Available online 22 July 2021

0921-8181/@ 2021 Elsevier B.V. All rights reserved. 
(continued)

\begin{tabular}{|c|c|c|c|}
\hline Taxon & Locality & Colector & Genebank \\
\hline Chrysophyllum boivinianum (Pierre) Baehni & Africa & McPherson 14,426 (MO, P, WAG) & DQ246667.1 \\
\hline Chrysophyllum bombycinum T.D. Penn. & Amazon & Serrano 118 (UDBC) & MZ329972 \\
\hline Chrysophyllum bombycinum T.D. Penn. & Amazon & Serrano 121 (UDBC) & MZ329973 \\
\hline Chrysophyllum bombycinum T.D. Penn. & Amazon & Serrano 120 (UDBC) & MZ329974 \\
\hline Chrysophyllum bombycinum T.D. Penn. & Amazon & Serrano 135 (UDBC) & MZ329975 \\
\hline Chrysophyllum bombycinum T.D. Penn. & Amazon & Serrano 139 (UDBC) & MZ329976 \\
\hline Chrysophyllum bombycinum T.D. Penn. & Amazon & Garcia $8671(\mathrm{E})$ & MZ470016 \\
\hline Chrysophyllum bombycinum T.D. Penn. & Amazon & Garcia $8673(\mathrm{E})$ & MZ470017 \\
\hline Chrysophyllum cainito $L$. & West Indies & Chantaranothai 2304 (KKU) & AY552153.1 \\
\hline Chrysophyllum cainito $L$. & Cauca Valley & Richardson et al. 357 (E) & MZ469952 \\
\hline Chrysophyllum colombianum (Aubrév.) T.D. Penn. & Chocó & Serrano 320 (UDBC) & MZ329873 \\
\hline Chrysophyllum colombianum (Aubrév.) T.D. Penn. & Chocó & Serrano 319 (UDBC) & MZ329874 \\
\hline Chrysophyllum colombianum (Aubrév.) T.D. Penn. & Amazon & Serrano 98 (UDBC) & MZ329970 \\
\hline Chrysophyllum colombianum (Aubrév.) T.D. Penn. & Amazon & Serrano 100 (UDBC) & MZ329971 \\
\hline Chrysophyllum colombianum (Aubrév.) T.D. Penn. & Chocó & Gentry, Faber-Langendoen \& Monsalve 56,806 (K) & MZ469957 \\
\hline Chrysophyllum cuneifolium (Rudge) A. DC. & Guianas & Mori et al. $19,135(\mathrm{~K})$ & DQ246668.1 \\
\hline Chrysophyllum eximium Ducke & Guianas & Baraloto 3135 (CAY) & FJ037866.1 \\
\hline Chrysophyllum fenerivense (Aubrév.) G.E. Schatz \& L. Gaut & Africa & Baraloto (CAY) & FJ037867 \\
\hline Chrysophyllum imperiale (Linden ex K. Koch \& Fintelm.) Benth. \& Hook. $f$. & Atlantic Forest & Pennington s.n. (S) & EF558615.1 \\
\hline Chrysophyllum $L$. & Amazon & Serrano 80 (UDBC) & MZ329882 \\
\hline Chrysophyllum L. & Amazon & Serrano 67 (UDBC) & MZ329958 \\
\hline Chrysophyllum $L$. & Amazon & Serrano 64 (UDBC) & MZ329959 \\
\hline Chrysophyllum $L$. & Amazon & Garcia $8647(\mathrm{E})$ & MZ469953 \\
\hline Chrysophyllum L. & Central America & Anderberg et al. $31^{\text {a }}$ & MZ470012 \\
\hline Chrysophyllum L. & Amazon & Garcia $8581(\mathrm{E})$ & MZ470013 \\
\hline Chrysophyllum lucentifolium Cronquist & Amazon & Serrano 49 (UDBC) & MZ329902 \\
\hline Chrysophyllum lucentifolium Cronquist & Amazon & Serrano 62 (UDBC) & MZ329904 \\
\hline Chrysophyllum manaosense (Aubrév.) T.D. Penn. & Chocó & Serrano 296 (UDBC) & MZ329875 \\
\hline Chrysophyllum manaosense (Aubrév.) T.D. Penn. & Amazon & S. coll. $2^{\mathrm{a}}$ & MZ468590 \\
\hline Chrysophyllum manaosense (Aubrév.) T.D. Penn. & Amazon & Richardson et al. 334 (E) & MZ469955 \\
\hline Chrysophyllum mexicanum Brandegee ex Standl. & Mexico & JP341 (DAV) & JF912989 \\
\hline Chrysophyllum mexicanum Brandegee ex Standl. & Mexico & JP398 (DAV) & JF912990 \\
\hline Chrysophyllum mexicanum Brandegee ex Standl. & Mexico & JP256 (DAV) & JF912991 \\
\hline Chrysophyllum oliviforme $L$. & West Indies & Guttiérrez \& Nilsson 1 (S) & DQ246670 \\
\hline Chrysophyllum pauciflorum Lam & West Indies & JP56 (DAV) & JF912986 \\
\hline Chrysophyllum pomiferum (Eyma) T.D. Penn. & Magdalena Valley & Pennington et al. $12,386^{\mathrm{a}}$ & MZ469956 \\
\hline Chrysophyllum prieurii A. DC. & Amazon & Richardson et al. 326 (E) & MZ469958 \\
\hline Chrysophyllum prieurii A. DC. & Amazon & Garcia $8583(\mathrm{E})$ & MZ469982 \\
\hline Chrysophyllum prieurii A. DC. & Guianas & Baraloto, Engel \& Chave s.n. (CAY) & P01860227 \\
\hline Chrysophyllum pruniforme Pierre ex Engl. & Africa & Jongkind 3762 (WAG) & DQ246671 \\
\hline Chrysophyllum roxburghii G. Don & Africa & Solo \& Randrianasolo 33 (WAG) & DQ246672 \\
\hline Chrysophyllum sanguinolentum (Pierre) Baehni & Guianas & Baraloto 3016 (CAY) & FJ037868 \\
\hline Chrysophyllum sanguinolentum (Pierre) Baehni & Guianas & Baraloto 3196 (CAY) & FJ037869.2 \\
\hline Chrysophyllum sanguinolentum (Pierre) Baehni & Amazon & Garcia $8645(E)$ & MZ469968 \\
\hline Chrysophyllum sanguinolentum (Pierre) Baehni & Amazon & Richardson et al. 305 (E) & MZ469980 \\
\hline Chrysophyllum sanguinolentum (Pierre) Baehni & Amazon & Richardson et al. 310 (E) & MZ470011 \\
\hline Chrysophyllum sanguinolentum (Pierre) Baehni & Amazon & Richardson et al. 348 (E) & MZ470015 \\
\hline Chrysophyllum sanguinolentum (Pierre) Baehni & Amazon & Garcia 8236 (E) & MZ470018 \\
\hline Chrysophyllum scalare T.D. Penn. & Amazon & Richardson et al. 320 (E) & MZ470014 \\
\hline Chrysophyllum sparsiflorum Klotzsch ex Miq. & Amazon & GUY71 $^{\mathrm{a}}$ & DQ021882 \\
\hline Chrysophyllum venezuelanense (Pierre) T.D. Penn. & Chocó & Ståhl et al. 5755 (S) & DQ246673 \\
\hline Diploon cuspidatum (Hoehne) Cronquist & Guianas & Pennington et al. $13,843(\mathrm{U})$ & DQ246676 \\
\hline Diploon cuspidatum (Hoehne) Cronquist & Guianas & Baraloto 3209 (CAY) & FJ037870 \\
\hline Eberhardtia aurata (Pierre ex Dubard) Lecomte & Asia & Hao $534(S)$ & EF558617 \\
\hline Ecclinusa guianensis Eyma & Amazon & Ducke 05-906 (K) & DQ246677 \\
\hline Ecclinusa lanceolata (Mart. \& Eichler) Pierre & Amazon & Richardson et al. 314 (E) & MZ470007 \\
\hline Ecclinusa lanceolata (Mart. \& Eichler) Pierre & Amazon & Garcia $8689(E)$ & MZ470008 \\
\hline Ecclinusa lanceolata (Mart. \& Eichler) Pierre & Amazon & Garcia $8675(\mathrm{E})$ & MZ470009 \\
\hline Ecclinusa Mart. & Chocó & Serrano 309 (UDBC) & MZ329966 \\
\hline Ecclinusa ramiflora Mart. & Guianas & Irwing et al. 55,081 (S) & DQ246678 \\
\hline Ecclinusa ramiflora Mart. & Macarena & Serrano 170 (UDBC) & MZ329964 \\
\hline Ecclinusa ramiflora Mart. & Macarena & Serrano 177 (UDBC) & MZ329965 \\
\hline Ecclinusa ramiflora Mart. & Macarena & Serrano nn $360^{\mathrm{a}}$ & MZ468579 \\
\hline Ecclinusa ramiflora Mart. & Macarena & Serrano nn361 ${ }^{\mathrm{a}}$ & MZ468580 \\
\hline Ecclinusa ramiflora Mart. & Chocó & Monsalve B1319 (K) & MZ470010 \\
\hline Elaeoluma Baill. & Amazon & Serrano 131 (UDBC) & MZ329963 \\
\hline Elaeoluma glabrescens (Mart. \& Eichler) Aubrév. & Central America & Anderberg et al. 33 (S) & EF558616 \\
\hline Elaeoluma schomburgkiana (Miq.) Baill. & Amazon & Keel \& Koelho 243 (S) & DQ246679 \\
\hline Elaeoluma schomburgkiana (Miq.) Baill. & Amazon & Keel \& Koelho s.n. (S) & MZ470006 \\
\hline Englerophytum magalismontanum (Sond.) T.D. Penn. & Africa & Swenson \& Karis 631 (S) & DQ246680 \\
\hline Englerophytum natalense (Sond.) T.D. Penn. & Africa & Kayombo 3483 (S) & AY552150 \\
\hline Manilkara bidentata (A. DC.) A. Chev & Magdalena Valley & Serrano 28 (UDBC) & MZ329856 \\
\hline Manilkara bidentata (A. DC.) A. Chev & Chocó & Serrano 294 (UDBC) & MZ329857 \\
\hline Manilkara bidentata (A. DC.) A. Chev & Chocó & Serrano 334 (UDBC) & MZ329858 \\
\hline Manilkara bidentata (A. DC.) A. Chev & Chocó & Serrano 245 (UDBC) & MZ329859 \\
\hline Micropholis (Griseb.) Pierre & Amazon & Alvarez $737^{\mathrm{a}}$ & MF574537 \\
\hline
\end{tabular}


(continued)

\begin{tabular}{|c|c|c|c|}
\hline Taxon & Locality & Colector & Genebank \\
\hline Micropholis (Griseb.) Pierre & Amazon & Alvarez $799^{\mathrm{a}}$ & MF574538 \\
\hline Micropholis (Griseb.) Pierre & Amazon & Sanchez et al. $22^{\mathrm{a}}$ & MF574539 \\
\hline Micropholis (Griseb.) Pierre & Amazon & Avila et al. 2733 (UDBC) & MF574540 \\
\hline Micropholis (Griseb.) Pierre & Amazon & Garcia $8618(E)$ & MZ470022 \\
\hline Micropholis brochidodroma T.D. Penn. & Amazon & JR-JH-1 & MF574503 \\
\hline Micropholis casiquiarensis Aubrév. & Amazon & Richardson et al. 340 (E) & MF574504 \\
\hline Micropholis cayennensis T.D. Penn. & Guianas & Baraloto, Engel \& Riera s.n. (CAY) & FJ037875.2 \\
\hline Micropholis crassipedicellata (Mart. \& Eichler ex Miq.) Pierre & Atlantic Forest & NH200222.a (CAY) & FJ037881 \\
\hline Micropholis crassipedicellata (Mart. \& Eichler ex Miq.) Pierre & Atlantic Forest & C.V. 171 (CEPEC) & JQ434164.1 \\
\hline Micropholis crassipedicellata (Mart. \& Eichler ex Miq.) Pierre & Atlantic Forest & C.V. 181 (CEPEC) & JQ434165.1 \\
\hline Micropholis crotonoides (Pierre) Pierre & Llanos & Ramirez et al. $50^{\mathrm{a}}$ & MF574506 \\
\hline Micropholis crotonoides (Pierre) Pierre & Llanos & ESR89 ${ }^{\mathrm{a}}$ & MF574507 \\
\hline Micropholis crotonoides (Pierre) Pierre & Llanos & JRM24 & MF574508 \\
\hline Micropholis egensis (A. DC.) Pierre & Amazon & Dionizia, Coêlho \& Ernesto 73 (U) & DQ246681 \\
\hline Micropholis egensis (A. DC.) Pierre & Guianas & Baraloto s.n. (CAY) & FJ037876 \\
\hline Micropholis egensis (A. DC.) Pierre & Amazon & Avila $2746^{\mathrm{a}}$ & MF574512 \\
\hline Micropholis egensis (A. DC.) Pierre & Amazon & Betancur, Echeverry, Kress \& Roesel 2848 (K) & MZ470021 \\
\hline Micropholis gardneriana (A. DC.) Pierre & Atlantic Forest & C.V. 177 (CEPEC) & JQ434158.1 \\
\hline Micropholis gardneriana (A. DC.) Pierre & Atlantic Forest & C.V. 205 (CEPEC) & JQ434159.1 \\
\hline Micropholis gardneriana (A. DC.) Pierre & Atlantic Forest & C.V. 70 (CEPEC) & JQ434160.1 \\
\hline Micropholis gardneriana (A. DC.) Pierre & Atlantic Forest & C.V. 215 (CEPEC) & JQ434161.1 \\
\hline Micropholis guyanensis (A. DC.) Pierre & West Indies & Taylor 11,691 (MO) & DQ246682 \\
\hline Micropholis guyanensis (A. DC.) Pierre & Guianas & Baraloto, Engel \& Riviera NH200477 (CAY) & FJ037877 \\
\hline Micropholis guyanensis (A. DC.) Pierre & Guianas & Baraloto et al. (CAY) & FJ037878.1 \\
\hline Micropholis guyanensis (A. DC.) Pierre & Atlantic Forest & C.V. 113 (CEPEC) & JQ434166.1 \\
\hline Micropholis guyanensis (A. DC.) Pierre & Atlantic Forest & C.V. 200 (CEPEC) & JQ434167.1 \\
\hline Micropholis guyanensis (A. DC.) Pierre & Atlantic Forest & C.V. 24 (CEPEC) & JQ434168.1 \\
\hline Micropholis guyanensis (A. DC.) Pierre & Atlantic Forest & ALCB $110909^{\mathrm{a}}$ & KF943856.1 \\
\hline Micropholis guyanensis (A. DC.) Pierre & Llanos & CR194 & MF574513 \\
\hline Micropholis guyanensis (A. DC.) Pierre & Llanos & Miranda et al. $93^{\mathrm{a}}$ & MF574514 \\
\hline Micropholis guyanensis (A. DC.) Pierre & Llanos & Miranda et al. $106^{\mathrm{a}}$ & MF574515 \\
\hline Micropholis guyanensis (A. DC.) Pierre & Amazon & Avila $2684^{\mathrm{a}}$ & MF574516 \\
\hline Micropholis guyanensis (A. DC.) Pierre & Amazon & Pineros et al. $14^{\mathrm{a}}$ & MF574517 \\
\hline Micropholis guyanensis (A. DC.) Pierre & Amazon & Richardson et al. 328 (E) & MF574518 \\
\hline Micropholis guyanensis (A. DC.) Pierre & Amazon & Serrano 68 (UDBC) & MZ329979 \\
\hline Micropholis guyanensis (A. DC.) Pierre & Amazon & Serrano 71 (UDBC) & MZ329980 \\
\hline Micropholis guyanensis (A. DC.) Pierre & Amazon & Serrano 53 (UDBC) & MZ329981 \\
\hline Micropholis guyanensis (A. DC.) Pierre & Amazon & Serrano 77 (UDBC) & MZ329985 \\
\hline Micropholis guyanensis (A. DC.) Pierre & Amazon & Serrano 66 (UDBC) & MZ329986 \\
\hline Micropholis guyanensis (A. DC.) Pierre & Amazon & Serrano 52 (UDBC) & MZ329987 \\
\hline Micropholis guyanensis (A. DC.) Pierre & Amazon & S. coll. $5^{\mathrm{a}}$ & MZ468592 \\
\hline Micropholis guyanensis (A. DC.) Pierre & Amazon & JR-PA-08 ${ }^{\mathrm{a}}$ & MZ468593 \\
\hline Micropholis guyanensis (A. DC.) Pierre & Guianas & S. coll. $3^{\mathrm{a}}$ & MZ468595 \\
\hline Micropholis guyanensis (A. DC.) Pierre & Amazon & Garcia $8620(\mathrm{E})$ & MZ470024 \\
\hline Micropholis guyanensis (A. DC.) Pierre & Amazon & Garcia $8242(\mathrm{E})$ & MZ470025 \\
\hline Micropholis guyanensis (A. DC.) Pierre & Amazon & Richardson s.n. (E) & MZ470026 \\
\hline Micropholis guyanensis (A. DC.) Pierre & Guianas & Baraloto 3107 (CAY) & NL110516 \\
\hline Micropholis humboldtiana (Roem. \& Schult.) T.D. Penn. & Amazon & PU72 (COAH) & MF574525 \\
\hline Micropholis longipedicellata Aubrév. & Guianas & Baraloto 3210 (CAY) & FJ037879 \\
\hline Micropholis longipedicellata Aubrév. & Atlantic Forest & Baraloto s.n. (CAY) & FJ037879.1 \\
\hline Micropholis longipedicellata Aubrév. & Guianas & Baraloto 3210a (CAY) & NL110459 \\
\hline Micropholis macrophylla (Krause) T.D. Penn. & Amazon & $\mathrm{EP} 102^{\mathrm{a}}$ & MF574528.1 \\
\hline Micropholis madeirensis (Baehni) Aubrév. & Amazon & Cortes et al. 2869 (UDBC) & MF574529 \\
\hline Micropholis madeirensis (Baehni) Aubrév. & Amazon & MS $3660^{\mathrm{a}}$ & MF574530 \\
\hline Micropholis madeirensis (Baehni) Aubrév. & Amazon & Richardson et al. 329 (E) & MF574531 \\
\hline Micropholis melinoniana Pierre & Amazon & Alvarez $1999^{\mathrm{a}}$ & MF574532 \\
\hline Micropholis obscura T.D. Penn. & Guianas & Baraloto 3218 (CAY) & FJ037880.1 \\
\hline Micropholis obscura T.D. Penn. & Amazon & S. coll. $4^{\mathrm{a}}$ & MZ468591 \\
\hline Micropholis obscura T.D. Penn. & Guianas & Baraloto 3218a (CAY) & NH200217 \\
\hline Micropholis obscura T.D. Penn. & Guianas & Baraloto 3217 (CAY) & P00610293 \\
\hline Micropholis porphyrocarpa (Baehni) Monach. & Guianas & Baraloto 3220 (CAY) & FJ037882.1 \\
\hline Micropholis porphyrocarpa (Baehni) Monach. & Guianas & Baraloto 3219 (CAY) & FJ037883.1 \\
\hline Micropholis splendens Gilly ex Aubrév. & Amazon & Avila et al. 2693 (UDBC) & MF574541 \\
\hline Micropholis trunciflora Ducke & Amazon & Cardenas $12266^{\mathrm{a}}$ & MF574542 \\
\hline Micropholis venulosa (Mart. \& Eichler) Pierre & Amazon & Assunção 122 (U) & DQ246683.1 \\
\hline Micropholis venulosa (Mart. \& Eichler) Pierre & Guianas & Baraloto (CAY) & FJ037884.1 \\
\hline Micropholis venulosa (Mart. \& Eichler) Pierre & Guianas & Baraloto (CAY) & FJ037885.1 \\
\hline Micropholis venulosa (Mart. \& Eichler) Pierre & Amazon & AD8893 (UDBC) & MF574543 \\
\hline Micropholis venulosa (Mart. \& Eichler) Pierre & Amazon & Avila et al. 2704 & MF574549 \\
\hline Micropholis venulosa (Mart. \& Eichler) Pierre & Llanos & Sanchez et al. $124^{\mathrm{a}}$ & MZ468594 \\
\hline Micropholis venulosa (Mart. \& Eichler) Pierre & Amazon & S. coll. $1^{\mathrm{a}}$ & MZ468596 \\
\hline Micropholis venulosa (Mart. \& Eichler) Pierre & Amazon & Garcia $8005(E)$ & MZ470020 \\
\hline Micropholis venulosa (Mart. \& Eichler) Pierre & Amazon & Garcia $8238(E)$ & MZ470023 \\
\hline Micropholis venulosa (Mart. \& Eichler) Pierre & Guianas & Baraloto 3128 (CAY) & NL110260 \\
\hline Micropholis venulosa (Mart. \& Eichler) Pierre & Guianas & Baraloto 3114 (CAY) & NL110297 \\
\hline Omphalocarpum pachysteloides Mildbr. ex Hutch. \& Dalziel & Africa & Jongkind 2351 (WAG) & AY552151 \\
\hline
\end{tabular}


(continued)

\begin{tabular}{|c|c|c|c|}
\hline Taxon & Locality & Colector & Genebank \\
\hline Omphalocarpum strombocarpum Y.B. Harv. \& Lovett & Africa & Frimodt-Møller et al. 538 (C) & DQ246685 \\
\hline Pouteria adolfi-friedericii (Engl.) A. Meeuse & Africa & Friis et al. 3502 (UPS) & AY552115 \\
\hline Pouteria alnifolia (Baker) Roberty & Africa & Jongkind \& Noyes 1322 (MO) & DQ246686 \\
\hline Pouteria altissima (A. Chev.) Baehni & Africa & Friis et al. 4145 (UPS) & DQ246687 \\
\hline Pouteria ambelaniifolia (Sandwith) T.D. Penn. & Guianas & Baraloto s.n. (CAY) & FJ037886 \\
\hline Pouteria Aubl. & Chocó & Serrano 244 (UDBC) & MZ329862 \\
\hline Pouteria Aubl. & Chocó & Serrano 306 (UDBC) & MZ329864 \\
\hline Pouteria Aubl. & Chocó & Serrano 246 (UDBC) & MZ329865 \\
\hline Pouteria Aubl. & Chocó & Serrano 247 (UDBC) & MZ329866 \\
\hline Pouteria Aubl. & Chocó & Serrano 301 (UDBC) & MZ329871 \\
\hline Pouteria Aubl. & Chocó & Serrano 338 (UDBC) & MZ329877 \\
\hline Pouteria Aubl. & Chocó & Serrano 267 (UDBC) & MZ329893 \\
\hline Pouteria Aubl. & Amazon & Serrano 54 (UDBC) & MZ329903 \\
\hline Pouteria Aubl. & Chocó & Serrano 312 (UDBC) & MZ329938 \\
\hline Pouteria Aubl. & Chocó & Serrano 307 (UDBC) & MZ329977 \\
\hline Pouteria Aubl. & Asia & Armstrong $317^{\mathrm{a}}$ & KF686303 \\
\hline Pouteria Aubl. & Guianas & Sabatier \& Molino Q423 ${ }^{\mathrm{a}}$ & MZ468562 \\
\hline Pouteria Aubl. & Guianas & Sabatier \& Molino $383(\mathrm{~K})$ & MZ468564 \\
\hline Pouteria Aubl. & Guianas & Sabatier \& Molino $213(\mathrm{P})$ & MZ468565 \\
\hline Pouteria Aubl. & Guianas & Sabatier \& Molino 989 (K) & MZ468567 \\
\hline Pouteria Aubl. & Guianas & Sabatier \& Molino V819 & MZ468569 \\
\hline Pouteria Aubl. & Amazon & S. coll. $8^{\mathrm{a}}$ & MZ468572 \\
\hline Pouteria Aubl. & Central America & Anderberg et al. 51 (S) & MZ469959 \\
\hline Pouteria Aubl. & Amazon & Garcia $8777(\mathrm{E})$ & MZ469963 \\
\hline Pouteria Aubl. & Amazon & Garcia $8632(\mathrm{E})$ & MZ469965 \\
\hline Pouteria Aubl. & Amazon & Garcia 8641 (E) & MZ469972 \\
\hline Pouteria Aubl. & Central America & Anderberg et al. 60 (S) & MZ469996 \\
\hline Pouteria Aubl. & Amazon & Garcia $8623(\mathrm{E})$ & MZ470019 \\
\hline Pouteria aubrevillei Bernardi & Amazon & Richardson et al. 327 (E) & MZ469969 \\
\hline Pouteria baehniana Monach & Amazon & Serrano 144 (UDBC) & MZ329860 \\
\hline Pouteria baehniana Monach & Amazon & Serrano 116 (UDBC) & MZ329861 \\
\hline Pouteria bangii (Rusby) T.D. Penn. & Catatumbo & Serrano 198 (UDBC) & MZ329932 \\
\hline Pouteria baueri (Montrouz.) Baehni & Australia & Munzinger 340 & AY552113 \\
\hline Pouteria benai (Aubrév. \& Pellegr.) T.D. Penn. & Guianas & Mori et al. 27,353 (K) & ED902434 \\
\hline Pouteria bilocularis (H.J.P. Winkl.) Baehni & Amazon & Richardson et al. 345 (E) & ED090242 \\
\hline Pouteria bilocularis (H.J.P. Winkl.) Baehni & Amazon & Garcia $8763(E)$ & MZ469995 \\
\hline Pouteria bracteata T.D. Penn. & Chocó & Serrano 298 (UDBC) & MZ329925 \\
\hline Pouteria bracteata T.D. Penn. & Chocó & Palacios 13,699 (K) & MZ469974 \\
\hline Pouteria caimito (Ruiz \& Pav.) Radlk. & Chocó & Serrano 318 (UDBC) & MZ329954 \\
\hline Pouteria caimito (Ruiz \& Pav.) Radlk. & Chocó & Serrano 311 (UDBC) & MZ329955 \\
\hline Pouteria caimito (Ruiz \& Pav.) Radlk. & Chocó & Serrano 305 (UDBC) & MZ329956 \\
\hline Pouteria caimito (Ruiz \& Pav.) Radlk. & Chocó & Serrano 299 (UDBC) & MZ329957 \\
\hline Pouteria caimito (Ruiz \& Pav.) Radlk. & Chocó & Richardson et al. 388 (E) & MZ469985 \\
\hline Pouteria caimito (Ruiz \& Pav.) Radlk. & Chocó & Richardson et al. 391 (E) & MZ469986 \\
\hline Pouteria caimito (Ruiz \& Pav.) Radlk. & Chocó & Richardson et al. 386 (E) & MZ469994 \\
\hline Pouteria caimito (Ruiz \& Pav.) Radlk. & Chocó & Richardson et al. 387 (E) & MZ469997 \\
\hline Pouteria caimito (Ruiz \& Pav.) Radlk. & Chocó & Richardson et al. 377 (E) & MZ469998 \\
\hline Pouteria caimito (Ruiz \& Pav.) Radlk. & Chocó & Richardson et al. 390 (E) & MZ469999 \\
\hline Pouteria campechiana (Kunth) Baehni & West Indies & Wang W00798 (HAST) & DQ246688 \\
\hline Pouteria collina (Little) T.D. Penn. & Chocó & Serrano 258 (UDBC) & MZ329948 \\
\hline Pouteria collina (Little) T.D. Penn. & Chocó & Serrano 263 (UDBC) & MZ329949 \\
\hline Pouteria collina (Little) T.D. Penn. & Chocó & Gentry, Faber-Langendoen \& Echevarria 62,908 (K) & MZ469973 \\
\hline Pouteria coriacea (Pierre) Pierre & Macarena & Serrano 165 (UDBC) & MZ329926 \\
\hline Pouteria coriacea (Pierre) Pierre & Macarena & Serrano 174 (UDBC) & MZ329927 \\
\hline Pouteria coriacea (Pierre) Pierre & Macarena & Serrano 168 (UDBC) & MZ329928 \\
\hline Pouteria coriacea (Pierre) Pierre & Macarena & Serrano 173 (UDBC) & MZ329929 \\
\hline Pouteria coriacea (Pierre) Pierre & Macarena & Serrano 169 (UDBC) & MZ329930 \\
\hline Pouteria coriacea (Pierre) Pierre & Macarena & Serrano 164 (UDBC) & MZ329931 \\
\hline Pouteria coriacea (Pierre) Pierre & Macarena & Serrano nn $365^{\mathrm{a}}$ & MZ468570 \\
\hline Pouteria coriacea (Pierre) Pierre & Macarena & Serrano nn $364^{\mathrm{a}}$ & MZ468571 \\
\hline Pouteria cuspidata (A. DC.) Baehni & Chocó & Serrano 337 (UDBC) & MZ329906 \\
\hline Pouteria cuspidata (A. DC.) Baehni & Catatumbo & Serrano 210 (UDBC) & MZ329907 \\
\hline Pouteria cuspidata (A. DC.) Baehni & Catatumbo & Serrano 211 (UDBC) & MZ329908 \\
\hline Pouteria cuspidata (A. DC.) Baehni & Catatumbo & Serrano 208 (UDBC) & MZ329909 \\
\hline Pouteria cuspidata (A. DC.) Baehni & Catatumbo & Serrano 205 (UDBC) & MZ329910 \\
\hline Pouteria cuspidata (A. DC.) Baehni & Catatumbo & Serrano 224 (UDBC) & MZ329911 \\
\hline Pouteria cuspidata (A. DC.) Baehni & Catatumbo & Serrano 207 (UDBC) & MZ329912 \\
\hline Pouteria cuspidata (A. DC.) Baehni & Catatumbo & Serrano 219 (UDBC) & MZ329913 \\
\hline Pouteria cuspidata (A. DC.) Baehni & Catatumbo & Serrano 215 (UDBC) & MZ329914 \\
\hline Pouteria cuspidata (A. DC.) Baehni & Catatumbo & Serrano 212 (UDBC) & MZ329915 \\
\hline Pouteria cuspidata (A. DC.) Baehni & Chocó & Serrano 242 (UDBC) & MZ329916 \\
\hline Pouteria cuspidata (A. DC.) Baehni & Catatumbo & Serrano 203 (UDBC) & MZ329917 \\
\hline Pouteria cuspidata (A. DC.) Baehni & Catatumbo & Serrano 209 (UDBC) & MZ329918 \\
\hline Pouteria cuspidata (A. DC.) Baehni & Catatumbo & Serrano 204 (UDBC) & MZ329919 \\
\hline Pouteria cuspidata (A. DC.) Baehni & Catatumbo & Serrano 193 (UDBC) & MZ329920 \\
\hline Pouteria cuspidata (A. DC.) Baehni & Catatumbo & Serrano 218 (UDBC) & MZ329921 \\
\hline
\end{tabular}


(continued)

\begin{tabular}{|c|c|c|c|}
\hline Taxon & Locality & Colector & Genebank \\
\hline Pouteria cuspidata (A. DC.) Baehni & Catatumbo & Serrano 185 (UDBC) & MZ329922 \\
\hline Pouteria cuspidata (A. DC.) Baehni & Catatumbo & Serrano 221 (UDBC) & MZ329923 \\
\hline Pouteria cuspidata (A. DC.) Baehni & Chocó & Serrano 336 (UDBC) & MZ329924 \\
\hline Pouteria cuspidata (A. DC.) Baehni & Chocó & Serrano 268 (UDBC) & MZ329933 \\
\hline Pouteria cuspidata (A. DC.) Baehni & Chocó & Serrano 335 (UDBC) & MZ329934 \\
\hline Pouteria cuspidata (A. DC.) Baehni & Chocó & Serrano 285 (UDBC) & MZ329935 \\
\hline Pouteria cuspidata (A. DC.) Baehni & Chocó & Serrano 269 (UDBC) & MZ329936 \\
\hline Pouteria cuspidata (A. DC.) Baehni & Chocó & Serrano 249 (UDBC) & MZ329937 \\
\hline Pouteria cuspidata (A. DC.) Baehni & Chocó & Serrano 321 (UDBC) & MZ329939 \\
\hline Pouteria cuspidata (A. DC.) Baehni & Macarena & Serrano 161 (UDBC) & MZ329940 \\
\hline Pouteria cuspidata (A. DC.) Baehni & Macarena & Serrano 159 (UDBC) & MZ329941 \\
\hline Pouteria cuspidata (A. DC.) Baehni & Macarena & Serrano 167 (UDBC) & MZ329942 \\
\hline Pouteria cuspidata (A. DC.) Baehni & Macarena & Serrano 166 (UDBC) & MZ329943 \\
\hline Pouteria cuspidata (A. DC.) Baehni & Macarena & Serrano 160 (UDBC) & MZ329944 \\
\hline Pouteria cuspidata (A. DC.) Baehni & Macarena & Serrano 156 (UDBC) & MZ329945 \\
\hline Pouteria cuspidata (A. DC.) Baehni & Macarena & Serrano 157 (UDBC) & MZ329946 \\
\hline Pouteria cuspidata (A. DC.) Baehni & Macarena & Serrano 162 (UDBC) & MZ329947 \\
\hline Pouteria cuspidata (A. DC.) Baehni & Catatumbo & Serrano nn $363^{\mathrm{a}}$ & MZ329982 \\
\hline Pouteria cuspidata (A. DC.) Baehni & Chocó & Serrano 284 (UDBC) & MZ329983 \\
\hline Pouteria cuspidata (A. DC.) Baehni & Chocó & Serrano 241 (UDBC) & MZ329984 \\
\hline Pouteria cuspidata (A. DC.) Baehni & Catatumbo & Serrano 158 (UDBC) & MZ468556 \\
\hline Pouteria cuspidata (A. DC.) Baehni & Catatumbo & Serrano 175 (UDBC) & MZ468573 \\
\hline Pouteria cuspidata (A. DC.) Baehni & Catatumbo & Serrano nn $368^{\mathrm{a}}$ & MZ468574 \\
\hline Pouteria cuspidata (A. DC.) Baehni & Catatumbo & Serrano nn $369^{\mathrm{a}}$ & MZ468575 \\
\hline Pouteria cuspidata (A. DC.) Baehni & Catatumbo & Serrano nn $366^{a}$ & MZ468576 \\
\hline Pouteria cuspidata (A. DC.) Baehni & Catatumbo & Serrano nn370 & MZ468577 \\
\hline Pouteria cuspidata (A. DC.) Baehni & Catatumbo & Serrano 176 (UDBC) & MZ468578 \\
\hline Pouteria cuspidata (A. DC.) Baehni & Amazon & Richardson et al. 337 (E) & MZ469976 \\
\hline Pouteria cuspidata (A. DC.) Baehni & Amazon & Garcia $8624(E)$ & MZ469977 \\
\hline Pouteria cuspidata (A. DC.) Baehni & Amazon & Garcia 8769 (E) & MZ469978 \\
\hline Pouteria decorticans T.D. Penn. & Guianas & Baraloto, Engel, Chave \& Riera s.n. (CAY) & FJ037887 \\
\hline Pouteria deliciosa T.D. Penn. & Guianas & Sabatier \& Molino $903^{\mathrm{a}}$ & MZ468563 \\
\hline Pouteria dominigensis (C.F. Gaertn.) Baehni & West Indies & Gutiérrez \& Nilsson 13 (S) & AY552106 \\
\hline Pouteria dominigensis (C.F. Gaertn.) Baehni & Magdalena Valley & Serrano 351 (UDBC) & MZ329876 \\
\hline Pouteria eugeniifolia (Pierre) Baehni & Guianas & Baraloto 3136 (CAY) & FJ037888 \\
\hline Pouteria eugeniifolia (Pierre) Baehni & Guianas & Baraloto, Engel, Chave \& Riera s.n. (CAY) & FJ037889.1 \\
\hline Pouteria filipes Eyma & Amazon & Serrano 140 (UDBC) & MZ329884 \\
\hline Pouteria filipes Eyma & Amazon & Serrano 73 (UDBC) & MZ329885 \\
\hline Pouteria gardneriana (A. DC.) Radlk. & Argentina & Schwarz 8216 (UPS) & DQ246689 \\
\hline Pouteria gongrijpii Eyma & Guianas & Baraloto 3087 (CAY) & FJ037890 \\
\hline Pouteria gongrijpii Eyma & Guianas & Baraloto 3098 (CAY) & FJ037891 \\
\hline Pouteria grandis Eyma & Guianas & Sabatier \& Molino $995^{\mathrm{a}}$ & MZ469960 \\
\hline Pouteria guianensis Aubl. & Guianas & Poncy $1745(\mathrm{P})$ & DQ246690 \\
\hline Pouteria guianensis Aubl. & Guianas & Baraloto \& Engel s.n. (CAY) & FJ037892 \\
\hline Pouteria guianensis Aubl. & Guianas & Baraloto, Engel, Chave \& Riera 3102 (CAY) & FJ037893 \\
\hline Pouteria guianensis Aubl. & Amazon & Richardson et al. 332 (E) & MZ469954 \\
\hline Pouteria guianensis Aubl. & Amazon & Richardson et al. 342 (E) & MZ469987 \\
\hline Pouteria guianensis Aubl. & Amazon & Richardson et al. 309 (E) & MZ469988 \\
\hline Pouteria guianensis Aubl. & Amazon & Richardson et al. 323 (E) & MZ469989 \\
\hline Pouteria guianensis Aubl. & Amazon & Garcia $8631(E)$ & MZ469990 \\
\hline Pouteria guianensis Aubl. & Amazon & Richardson et al. 351 (E) & MZ469991 \\
\hline Pouteria guianensis Aubl. & Amazon & Richardson et al. 333 (E) & MZ469992 \\
\hline Pouteria hispida Eyma & Guianas & Mori et al. 25,432 (NY) & DQ246691 \\
\hline Pouteria laevigata (Mart.) Radlk. & Chocó & Serrano 339 (UDBC) & MZ329960 \\
\hline Pouteria laevigata (Mart.) Radlk. & Central America & Anderberg et al. 49 (S) & MZ470001 \\
\hline Pouteria lecythidicarpa P.E. Sánchez \& Poveda & Magdalena Valley & Serrano 24 (UDBC) & MZ329878 \\
\hline Pouteria lecythidicarpa P.E. Sánchez \& Poveda & Magdalena Valley & Serrano $352^{\mathrm{a}}$ & MZ329879 \\
\hline Pouteria lecythidicarpa P.E. Sánchez \& Poveda & Magdalena Valley & Serrano 18 (UDBC) & MZ329880 \\
\hline Pouteria lecythidicarpa P.E. Sánchez \& Poveda & Magdalena Valley & Serrano 44 (UDBC) & MZ329881 \\
\hline Pouteria lecythidicarpa P.E. Sánchez \& Poveda & Central America & Anderberg et al. 34 (S) & MZ469961 \\
\hline Pouteria lucumifolia (Reissek ex Maxim.) T.D. Penn. & Amazon & Richardson et al. 306 (E) & MZ469975 \\
\hline Pouteria macrocarpa (Mart.) D. Dietr. & Guianas & Baraloto, Engel, Chave \& Riera 3095 (CAY) & FJ037894 \\
\hline Pouteria macrophylla (Lam.) Eyma & Amazon & Seidel et al. $5905(\mathrm{~K})$ & DQ246692 \\
\hline Pouteria multiflora (A. DC.) Eyma & Chocó & Villa \& Rivaz 257 (BM) & DQ246693 \\
\hline Pouteria oblanceolata Pires & Amazon & Richardson et al. 307 (E) & MZ470002 \\
\hline Pouteria oblanceolata Pires & Amazon & Garcia $8625(E)$ & MZ470003 \\
\hline Pouteria oblanceolata Pires & Amazon & Garcia 8635 (E) & MZ470004 \\
\hline Pouteria oblanceolata Pires & Amazon & Garcia 8634 (E) & MZ470005 \\
\hline Pouteria platyphylla (A.C. Sm.) Baehni & Chocó & Serrano 303 (UDBC) & MZ329870 \\
\hline Pouteria platyphylla (A.C. Sm.) Baehni & Magdalena Valley & Serrano $359^{\mathrm{a}}$ & MZ329892 \\
\hline Pouteria platyphylla (A.C. Sm.) Baehni & Catatumbo & Serrano 223 (UDBC) & MZ329894 \\
\hline Pouteria platyphylla (A.C. Sm.) Baehni & Magdalena Valley & Serrano $348^{\mathrm{a}}$ & MZ329895 \\
\hline Pouteria platyphylla (A.C. Sm.) Baehni & Magdalena Valley & Serrano $350^{\mathrm{a}}$ & MZ329896 \\
\hline Pouteria platyphylla (A.C. Sm.) Baehni & Amazon & Serrano 55 (UDBC) & MZ329897 \\
\hline Pouteria platyphylla (A.C. Sm.) Baehni & Amazon & Richardson et al. 313 (E) & MZ469964 \\
\hline Pouteria procera (Mart.) K. Hammer & Amazon & Richardson et al. 339 (E) & MZ469966 \\
\hline
\end{tabular}


(continued)

\begin{tabular}{|c|c|c|c|}
\hline Taxon & Locality & Colector & Genebank \\
\hline Pouteria pubescens (Aubrév. \& Pellegr.) T.D. Penn. & Amazon & S. coll. $6^{\mathrm{a}}$ & MZ468566 \\
\hline Pouteria pubescens (Aubrév. \& Pellegr.) T.D. Penn. & Amazon & Garcia $8636(E)$ & MZ469962 \\
\hline Pouteria putamen-ovi T.D. Penn. & Amazon & S. coll. $7^{\mathrm{a}}$ & MZ468568 \\
\hline Pouteria putamen-ovi T.D. Penn. & Amazon & Garcia $8774(E)$ & MZ469970 \\
\hline Pouteria resinosa T.D. Penn. & Chocó & Serrano 270 (UDBC) & MZ329886 \\
\hline Pouteria resinosa T.D. Penn. & Chocó & Serrano 259 (UDBC) & MZ329887 \\
\hline Pouteria resinosa T.D. Penn. & Chocó & Serrano 243 (UDBC) & MZ329889 \\
\hline Pouteria reticulata (Engl.) Eyma & Amazon & Serrano 72 (UDBC) & MZ329951 \\
\hline Pouteria reticulata (Engl.) Eyma & Central America & Anderberg et al. 7 (S) & MZ469993 \\
\hline Pouteria rostrata (Huber) Baehni & Magdalena Valley & Serrano 357 (UDBC) & MZ329891 \\
\hline Pouteria sessilis T.D. Penn. & Amazon & Garcia 8637 (E) & MZ469951 \\
\hline Pouteria sessilis T.D. Penn. & Amazon & Richardson et al. 321 (E) & MZ469983 \\
\hline Pouteria subrotata Cronquist & Amazon & Serrano 145 (UDBC) & MZ329967 \\
\hline Pouteria subrotata Cronquist & Amazon & Serrano 151 (UDBC) & MZ329968 \\
\hline Pouteria subrotata Cronquist & Amazon & Serrano 112 (UDBC) & MZ329969 \\
\hline Pouteria subrotata Cronquist & Central America & Anderberg et al. $48(\mathrm{~S})$ & MZ470000 \\
\hline Pouteria torta (Mart.) Radlk. & Guianas & Baraloto, Engel \& Riera s.n. (CAY) & FJ037895 \\
\hline Pouteria torta (Mart.) Radlk. & Amazon & Serrano 90 (UDBC) & MZ329863 \\
\hline Pouteria torta (Mart.) Radlk. & Amazon & Serrano 58 (UDBC) & MZ329883 \\
\hline Pouteria torta (Mart.) Radlk. & Amazon & Serrano 70 (UDBC) & MZ329898 \\
\hline Pouteria torta (Mart.) Radlk. & Amazon & Serrano 59 (UDBC) & MZ329899 \\
\hline Pouteria torta (Mart.) Radlk. & Amazon & Serrano 105 (UDBC) & MZ329900 \\
\hline Pouteria torta (Mart.) Radlk. & Amazon & Serrano 78 (UDBC) & MZ329901 \\
\hline Pouteria torta (Mart.) Radlk. & Amazon & Serrano 153 (UDBC) & MZ329961 \\
\hline Pouteria torta (Mart.) Radlk. & Amazon & Serrano 50 (UDBC) & MZ329962 \\
\hline Pouteria torta (Mart.) Radlk. & Amazon & Serrano 69 (UDBC) & MZ329978 \\
\hline Pouteria torta (Mart.) Radlk. & Amazon & Guy $55^{\mathrm{a}}$ & MZ468588 \\
\hline Pouteria torta (Mart.) Radlk. & Amazon & Richardson et al. 338 (E) & MZ469967 \\
\hline Pouteria torta (Mart.) Radlk. & Amazon & Anderberg et al. 47 (S) & MZ469981 \\
\hline Pouteria torta (Mart.) Radlk. & Amazon & Richardson et al. 336 (E) & MZ469984 \\
\hline Pouteria triplarifolia Standl. \& L.O. Williams ex T.D. Penn. & Central America & Swenson et al. 738 (S) & MZ469971 \\
\hline Pouteria venosa (Mart.) Baehni & Amazon & Serrano 149 (UDBC) & MZ329952 \\
\hline Pouteria venosa (Mart.) Baehni & Amazon & Serrano 76 (UDBC) & MZ329953 \\
\hline Pouteria vernicosa T.D. Penn. & Chocó & Villa et al. 1304 (BM) & DQ246694 \\
\hline Pouteria viridis (Pittier) Cronquist & Central America & Faber-Langendoen \& Renteria $1127(\mathrm{~K})$ & MZ468561 \\
\hline Pradosia atroviolacea Ducke & Central America & Anderberg et al. 52 (S) & KM042306 \\
\hline Pradosia atroviolacea Ducke & Chocó & Cuatrecasas $13,988(\mathrm{~K})$ & KM042310 \\
\hline Pradosia brevipes (Pierre) T.D. Penn. & Amazon & Lindeman $6743(\mathrm{U})$ & AY552158 \\
\hline Pradosia cuatrecasasii (Aubrév.) T.D. Penn. & Chocó & Baraloto, Engel \& Riera s.n. (CAY) & FJ037897 \\
\hline Pradosia Liais & Amazon & Serrano 56 (UDBC) & MZ329872 \\
\hline Pradosia ptychandra (Eyma) T.D. Penn. & Guianas & Baraloto, Engel, Chave \& Riera 3111 (CAY) & FJ037898 \\
\hline Pradosia ptychandra (Eyma) T.D. Penn. & Guianas & Baraloto, Engel, Chave \& Riera s.n. (CAY) & FJ037899.1 \\
\hline Pradosia ptychandra (Eyma) T.D. Penn. & Guianas & Baraloto, Engel, Chave \& Riera 3111 (CAY) & MZ468557 \\
\hline Pradosia schomburgkiana (A. DC.) Cronquist & Amazon & Ducke Reserve 05-1829 (K) & AY552156 \\
\hline Pradosia spinosa Ewango \& Breteler & Africa & Isembe 508 (WAG) & DQ246695 \\
\hline Pradosia surinamensis (Eyma) T.D. Penn. & Guianas & Harris $1076(\mathrm{U})$ & AY552157 \\
\hline Sapotaceae Juss. & Guianas & Guy $37^{\mathrm{a}}$ & FJ037896 \\
\hline Sapotaceae Juss. & Chocó & Serrano 288 (UDBC) & MZ329888 \\
\hline Sapotaceae Juss. & Chocó & Serrano 278 (UDBC) & MZ329890 \\
\hline Sapotaceae Juss. & Chocó & Serrano 315 (UDBC) & MZ329905 \\
\hline Sapotaceae Juss. & Magdalena Valley & Serrano 38 (UDBC) & MZ468558 \\
\hline Sapotaceae Juss. & Magdalena Valley & Serrano 39 (UDBC) & MZ468559 \\
\hline Sapotaceae Juss. & Guianas & Guy $24^{\mathrm{a}}$ & MZ468581 \\
\hline Sapotaceae Juss. & Guianas & Guy $3^{\mathrm{a}}$ & MZ468582 \\
\hline Sapotaceae Juss. & Guianas & Guy $76^{a}$ & MZ468583 \\
\hline Sapotaceae Juss. & Guianas & Guy $13^{\mathrm{a}}$ & MZ468584 \\
\hline Sapotaceae Juss. & Guianas & Guy $2^{\mathrm{a}}$ & MZ468585 \\
\hline Sapotaceae Juss. & Guianas & Guy $74^{\mathrm{a}}$ & MZ468586 \\
\hline Sapotaceae Juss. & Guianas & Guy $47^{\mathrm{a}}$ & MZ468587 \\
\hline Sapotaceae Juss. & Guianas & Guy $32^{\mathrm{a}}$ & MZ468589 \\
\hline Sarcaulus brasiliensis (A. DC.) Eyma & Amazon & Paniagua et al. 4852 (MO) & DQ246696 \\
\hline Sarcaulus brasiliensis (A. DC.) Eyma & Magdalena Valley & Serrano $355^{\mathrm{a}}$ & MZ329950 \\
\hline Sarcaulus brasiliensis (A. DC.) Eyma & Amazon & Richardson et al. 322 (E) & MZ469979 \\
\hline Sarcosperma laurinum (Benth.) Hook. $f$. & Asia & Saunders s.n. (S) & AM408055 \\
\hline Synsepalum dulcificum (Schumach. \& Thonn.) Daniell & Africa & Welsing et al. 24 (WAG) & DQ246697 \\
\hline Synsepalum passargei (Engl.) T.D. Penn. & Africa & Magogo 2452 (UPS) & DQ246698 \\
\hline Xantolis siamensis (Fletcher) P. Royen & Asia & Smitairi 1 (L) & AY552154.1 \\
\hline
\end{tabular}

a Sterile specimens not deposited at official herbarium collections.

\section{Acknowledgements}

We thank Michelle Hart and Laura Forest for laboratory assistance at the Royal Botanic Garden Edinburgh. Fabio Ávila and Doris Huertas are thanked for their assistance at the Herbario Forestal. Terry Pennington and Felix Forest at the Royal Botanic Gardens, Kew are thanked for providing samples of Colombian Sapotaceae and permitting their extraction respectively. Alain Franc supplied samples from French Guiana that were collected by Daniel Sabatier and Jean-François Molino all of whom are thanked for those contributions. Investissement 
d'Avenir" grants managed by Agence Nationale de la Recherche (CEBA, ref. ANR-10-LABX-25-01) is thanked for their financial to the work carried out by Jérôme Chave's Laboratory. Unpublished data was provided by Dayana Sanchez from the Universidad Distrital Francisco José de Caldas, and by Roosevelt Garcia from the Cornell University. The work was supported by a University of Edinburgh Darwin Trust PhD Scholarship awarded to the first author.

The authors would like to apologise again for any inconvenience caused. 